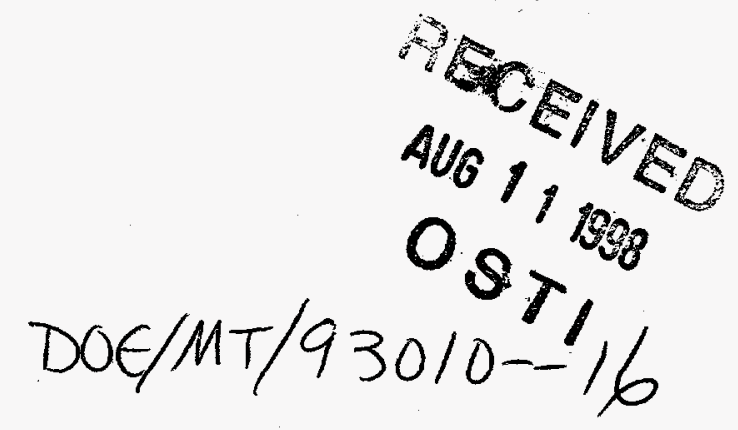

\title{
Investigation of Syngas Interactions in Alcohol Synthesis Catalysts
}

\author{
Technical Report \\ April 15, 1998
}

\author{
By: \\ Murty A. Akundi
}

Work Performed Under Contract No.: DE-FG22-93MT93010

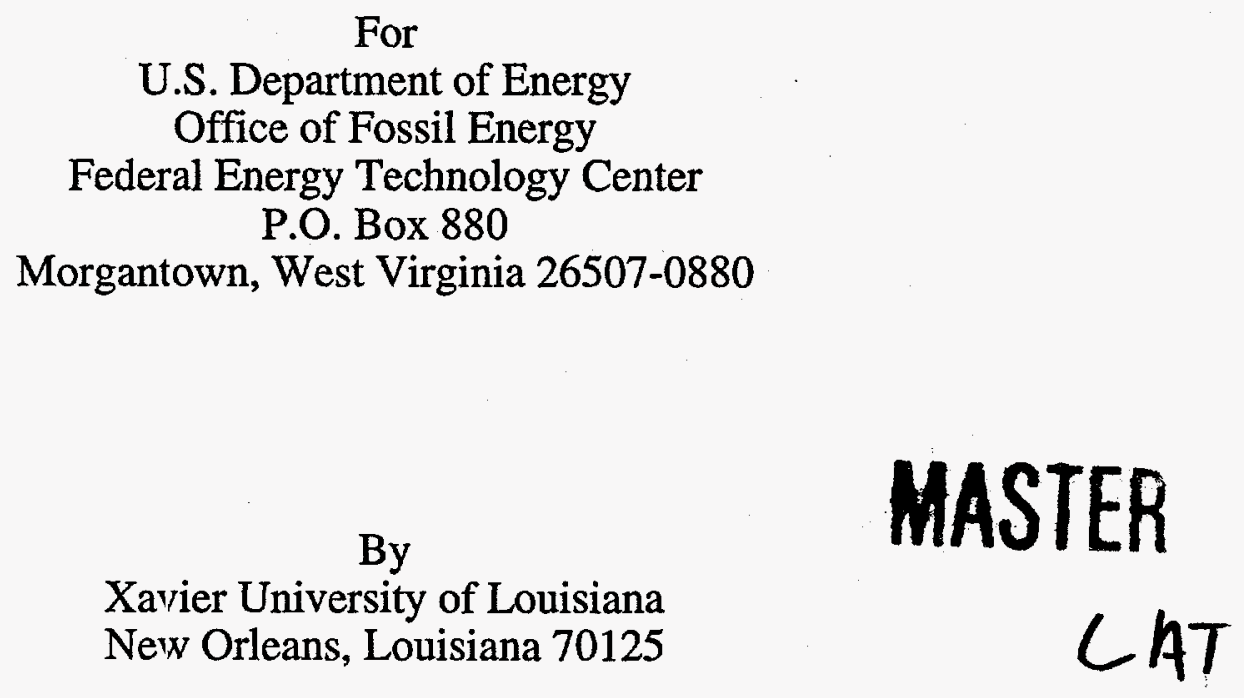




\section{Disclaimer}

This report was prepared as an account of work sponsored by an agency of the United States Government. Neither the United States Government nor any agency thereof, nor any of their employees, makes any warranty, express or implied, or assumes any legal liability or responsibility for the accuracy, completeness, or usefulness of any information, apparatus, product, or process disclosed, or represents that its use would not infringe privately owned rights. Reference herein to any specific commercial product, process, or service by trade name, trademark, manufacturer, or otherwise does not necessarily constitute or imply. its endorsement, recommendation, or favoring by the United States Government or any agency thereof. The views and opinions of authors expressed herein do not necessarily state or reflect those of the United States Government or any agency thereof. 


\section{DISCLAIMER}

Portions of this document may be illegible electronic image products. Images are produced from the best available original document. 


\section{TABLE OF CONTENTS}

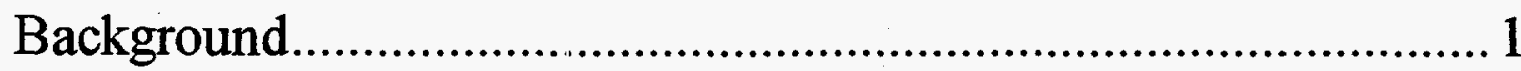

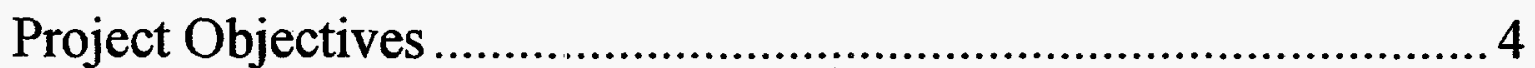

Experimental: Instrumental
A. Zero Field NMR
B. Magnetometry (VSM)
C. FTIR

Work Performed: Results and Discussion

A. Sample preparation

B. Effect of Precipitation Sequence

1. NMR Results and Magnetization Data

2. FTIR Studies

3. Catalytic Studies

36

C. Syngas Interaction Studies

1. NMR Results and Magnetization Data

2. FTIR Studies.

References

Minority Undergraduate Student Training: Papers Presented 74 reprints removed 


\section{BACKGROUND}

Catalysts comprised of dissimilar metals, specifically the early and the late transition elements, exhibit unusual activity and selectivity in the conversion of synthesis gas $\left(\mathrm{CO}+\mathrm{H}_{2}\right)$ to fuel alcohols and gasoline range hydrocarbons. Method of preparation, nature of support, and presence of other elements in the catalyst composite are known to govern the behavior of the catalyst. Though much experimental data on catalytic yields are available, little effort has been devoted to correlating the physical and chemical characteristics of these intermetallic composites. Very little is understood about the specific roles of the different elements in a catalyst and the underlying mechanisms in achieving the distinct functions of association, dissociation, and insertion of syngas for the production of alcohols.

Bimetallic catalysts have been receiving considerable attention in recent years by virtue of their potential for converting synthesis gas to mixed alcohols. The continued threat of oil shortages and the consequent effects on national and global economies make these studies critical [1-3]. Stringent regulations on the percentage of methanol in gasoline blends increase the attractiveness and incentive to develop processes for the efficient production of longer chain alcohols; which in turn can be blended in greater proportion with gasoline. Blended fuels are not only cleaner sources of energy but also improve driveability and boost octane number.

The catalyst systems developed and patented by several investigators [4-8] differ widely in method of preparation and composition of constituent elements. A wide variety of preparation techniques, combinations of transition elements, alkali metals, and supports seem to have the potential to fulfill the functionality requirements for production of 
alcohols. It is believed that dissimilar transition metals distributed at atomic distances function synergetically, accomplishing three functions: dissociation (C-O bond breaking), association (hydrogenation/chain growth), and insertion (CO insertion/hydrogenation) [9]. The effectiveness of the catalyst seems to be influenced by the composition, size, and morphology of the metal cluster. Cluster physics reveals [10] that small aggregates of metal atoms behave like "giant atoms" with electron energy levels that exhibit a "shell structure" similar to atomic nuclei. The number of atoms and the nature of interactions between the cluster atoms determine the energy of the delocalized electrons. These itinerant electrons are believed to be responsible for the chemical and magnetic properties of the cluster. Since the position and nature of the constituent atomic cores determine the number of itinerant electrons, the cluster character and consequently the behavior of the composite catalyst is sensitive to the method of preparation. The $\mathrm{pH}$, temperature, sequence of precipitation, drying, calcination, and reduction temperatures are known to influence the structure and properties of the catalyst. Keeping all other parameters constant, a detailed investigation of the effect of sequence of precipitation on the catalyst character is carried out in this study.

Although extensive valuable information is available on catalytic yields such as conversion efficiency, selectivity, product distribution, etc., relatively few studies have been made to analyze the nature of interactions between the transition metal atom and carbon monoxide/hydrogen gas molecules. A complete description of gas-metal interactions requires information both on the adsorbate and adsorbent simultaneously. In this investigation we examined the changes in the magnetic character of the ferromagnetic metal due to $\mathrm{CO}$ adsorption by Zero-field NMR of cobalt, magnetization study of the 
composite catalyst by Vibrating Sample Magnetrometry (VSM) and FTIR study of the adsorbed $\mathrm{CO}$ molecule. Many questions regarding these catalysts need to be answered. For example, does an electronic interaction occur between the ferromagnetic metal $(\mathrm{Co} / \mathrm{Fe})$ and copper or chromium /zinc such that there is an enhancement of the electron density at the transition metal surface thus changing the strength of the metal $-\mathrm{CO} / \mathrm{H}_{2}$ bonds? Is a particular crystalline phase such as hexagonal close packed (hcp) cobalt or face centered cubic ( $\mathrm{fcc}$ ) cobalt favored based on the preparative techniques. Is a rhombohedric phase of hydro-talcite structure or spinel structure or oxidic matrix structure predominate in the catalyst system? Does a change in the intermetallic ratio alter the strength and character of $\mathrm{CO} / \mathrm{H}_{2}$ adsorption? The techniques employed in this work, both ZFNMR and magnetization studies are sensitive to the crystalline phase and to changes in the electronic charge distribution around the transition metal nuclei and provide information on the adsorbent metal atom. The FTIR studies provide information on the adsorbate gas molecule and the changes in the stretching frequencies of the adsorbate reveal the nature of gas-metal interactions in the catalyst. The main focus of this study is to examine the subtle changes that occur in the character of adsorbate and adsorbent due to gas-metalintermetallic interactions and their relation to the observed selectivity character of the catalyst. 


\section{PROJECT OBJECTIVES}

The primary objectives of the project are to

a) synthesize, by controlled sequential and co-impregnation techniques, three distinct composition metal clusters (consisting of $\mathrm{Cu}-\mathrm{Co}-\mathrm{Cr}$ and $\mathrm{Cu}-\mathrm{Fe}-\mathrm{Zn}$ ): rich in copper (Methanol selective), rich in ferromagentic metal (Co or Fe - Hydrocarbon selective) and intermediate range (mixed alcohol catalysts);

b) investigate the changes in the magnetic character of the systems due to interaction with $\mathrm{CO}$, through

i) Zero-field Nuclear Magnetic Resonance (ZFNMR) study of cobalt

ii) Magnetic character (saturation magnetization and coercive field) analysis of the composite catalyst by Vibrating Sample Magnetometry (VSM);

c) examine the changes in syngas adsorption character of the catalyst as the composition changes, by FTIR Spectroscopic analysis of CO stretching frequencies;

d) determine the nature and size of these intermetallic clusters by Scanning Electron Microscopy (SEM); and

e) perform catalytic runs on selected samples and analyze the correlations between the physical and chemical characteristics.

The catalysts chosen have a greater promise for industrial application than the $\mathrm{Rh}$ and Mo based catalysts. Several groups preparing catalysts [4-9] by synthetic routes have reported divergent results for activity and selectivity. Generally the research has followed an empirical path and less effort is devoted to analyze the mechanisms and the scientific 
basis. The primary intent of this study is to analyze the nature of the intermetallic and gasmetal interactions and examine the correlations to catalytic properties. 


\section{EXPERIMENTAL: INSTRUMENTAL}

\section{A. Zero-field NMR Studies}

Since its discovery by Gossard and Portis in 1959, nuclear magnetic resonance in ferromagnetic materials has provided information concerning hyperfine fields, and nuclear spin relaxation. Using this technique it is possible to characterize multi-domain, and superparamagnetic particles [11].

At the nuclei of atoms of ferro-, antiferro-, and ferrimagnetic materials there exists a strong internal magnetic field due to the partial polarization of s-electrons by the magnetic state of the material. This field, referred to as the hyperfine field, is the same field as described in Mossbauer spectroscopy. The interaction of the nuclear spin with the hyperfine field gives rise to a set of quantized energy states which lead to the well-known resonance condition $\omega=\gamma \mathbf{H}_{\mathbf{h f}}$ where $\gamma$ is the nuclear gyromagnetic ratio and $\mathbf{H}_{\mathbf{h f}}$ is the hyperfine field. Thus, resonance conditions exist without the application of an external magnetic field, and through resonance techniques the internal field can be determined. It should be noted that the polarization of the s-electrons is a result of the electrostatic coupling of $3 \mathrm{~d}$ (or $4 \mathrm{f}$ ) electrons and s-electrons (both inner and outer s-band). As such the hyperfine field reflects the $3 \mathrm{~d}$ electronic structure and behavior, and can provide valuable information if significant perturbations in the charge distribution occur due to syngas adsorption on the ferromagnetic metal, or because of intermetallic interactions with in the metal cluster due to compositional changes or preparative techniques.

Adsorption of a molecule on the surface of ferromagnetic solid produces a change in the magnetization of the solid. If the adsorption process involves appreciable electronic 
interaction and if the ratio of surface to volume in the adsorbent is large, then the fractional change of magnetization becomes substantial and lends itself to convenient measurement and interpretation [12]. The NMR line frequency can be represented by $\omega=\gamma\left(\mathbf{H h f}_{\mathbf{h f}} \pm \Delta \mathbf{H}_{\mathbf{h f}}\right)$ where $\Delta$. $\mathbf{H}_{\mathbf{h f}}$ represents the resulting modifications in the charge distribution in the vicinity of the cobalt nucleus due to the adsorbed molecule.

Figure 1 is a block diagram of the NQR/ZFNMR spectrometer system. The spectrometer is a conventional Superregenerative oscillator (SRO) with Zeeman modulation and phase sensitive detection. It was designed by WILK's scientific corporation as a Nuclear Quadrupole Resonance spectrometer with a servo controlled self quenching system for auto scan operation in the frequency range $2-350 \mathrm{MHz}$. Since Zeeman modulation is not suitable for ferromagnetic materials, frequency modulation was employed. This was easily accomplished using a woofer (low frequency) speaker to vibrate a small metal strip near the oscillator tank circuit.

The spectra were scanned in the $205-230 \mathrm{MHz}$ region. The carrier frequency of the SRO was determined by displaying the oscillator output on a Tektronix Model 2712 spectrum analyzer/frequency counter. The NMR spectra were scanned at a flow rate of $0.1 \mathrm{MHz} / \mathrm{min}$ and the resonance frequencies were ascertained with an accuracy of \pm 0.3 MHz. A typical NMR spectrum of a catalyst sample is shown in Figure 2. 


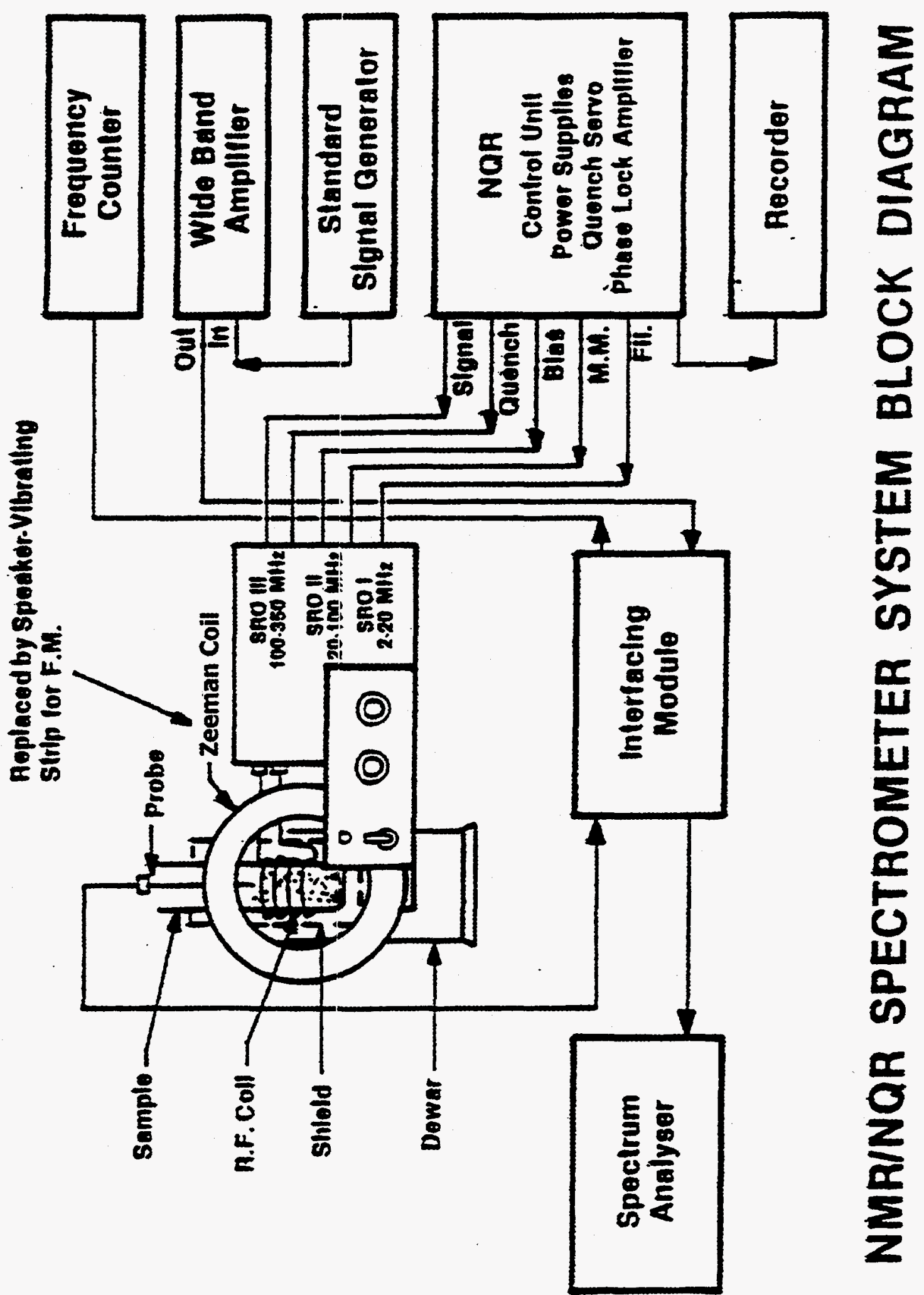




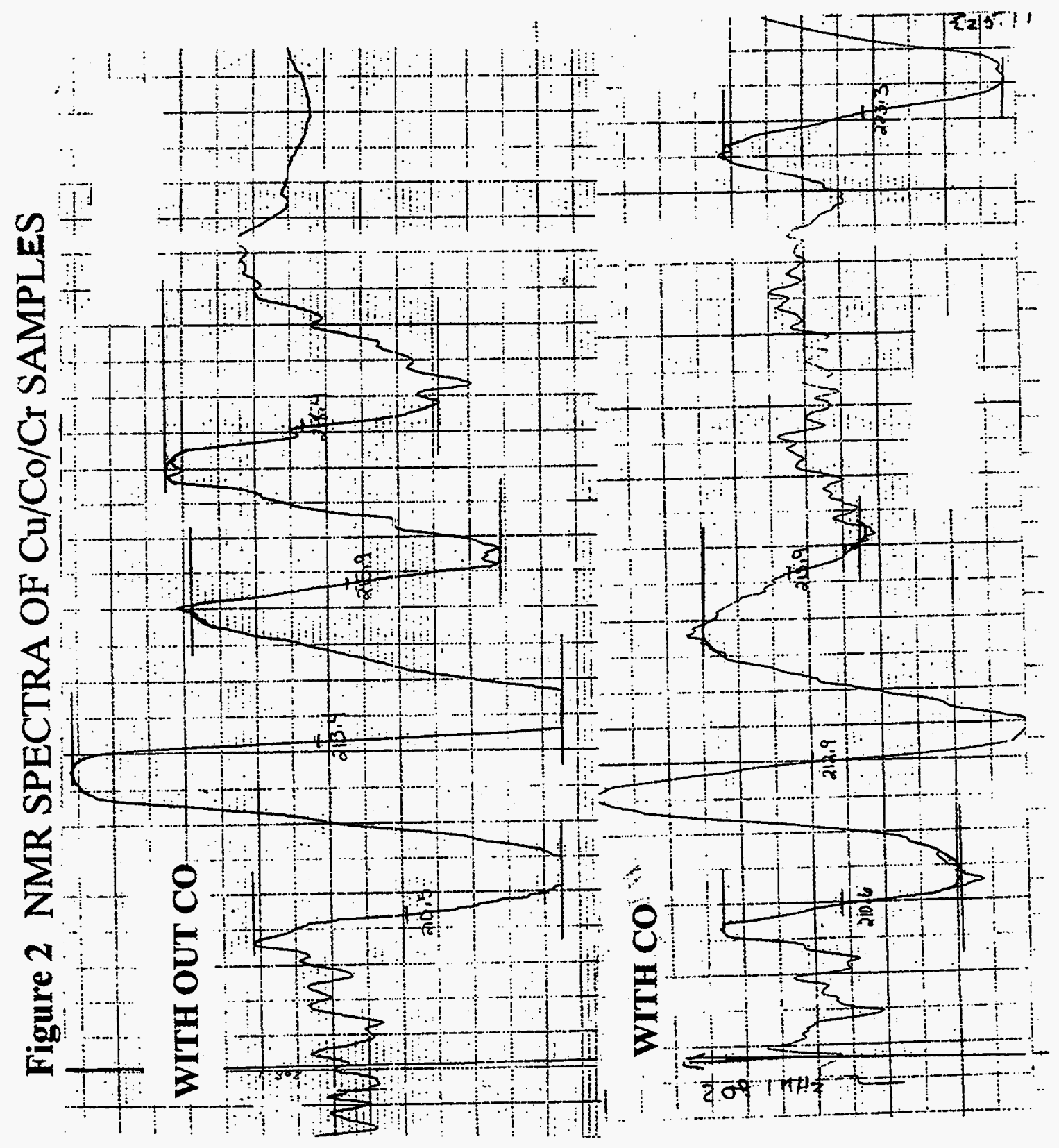




\section{B. Magnetic Measurements}

Substances composed of atoms or ions with permanent magnetic moments arising from unpaired electron spins can be classified as para-, ferro-, antiferro-, or ferrimagnetic depending on such factors as exchange interaction between neighboring species, chemical composition, crystalline structure, crystal field effects, particle size, and temperature [13].

For paramagnetic behavior the magnetic susceptibility $(\chi)$ defined as the ratio of magnetization $(\mathrm{M})$ to Field $(\mathrm{H})$, can be adequately described by the Curie Weiss law:

$\chi=C /(T-\theta)$ where $C$ and $\theta$ are the Curie and Weiss constants. When neighboring atoms or groups of atoms interact strongly and behave in a cooperative fashion, a ferromagnetic state exists with all spins 'parallel' in its domain.

Highly dispersed metals with metal crystallite sizes of less than single magnetic domain size exhibit unique magnetic properties [14]. Although the atoms which comprise these single domain particles are ferromagnetically coupled, the particles themselves behave like paramagnetic particles with very large magnetic moments. This gives rise to a behavior known as super paramagnetism. The magnetic moment associated with these superparamagnetic particles is directly proportional to the particle volume. The particle size can be determined from magnetization measurements [15]. Such analysis has been used to determine average particle size and particle size distribution for many highly dispersed metal catalysts [16-17]. Most heterogeneous catalysts are comprised of transition elements, their oxides or compounds, and often include various supports. The important characteristics of the transition elements are their incomplete d-electron shell and their unpaired electron spins. These features are responsible for their specific magnetic as well as their valuable catalytic properties. 
Significant changes in the saturation magnetization $M_{s}$ have been reported for a number of ferromagnetic catalysts due to chemisorption of $\mathrm{H}_{2}\left(\mathrm{H}_{2} / \mathrm{Ni}-\mathrm{Cu}, \mathrm{H}_{2} / \mathrm{Co}, \mathrm{H}_{2} / \mathrm{Fe}\right)$. The change in magnetic moment per adsorbate molecule, $\varepsilon$, in general can be represented by , where $\Delta \mathbf{M}_{\mathbf{s}}$ change in saturation magetization, $\mathbf{M}_{\mathbf{s}}$ saturation magnetization, $\mathrm{n}_{\text {metal }}$ number of moles of the metal or gas, and $\beta$ is the Bohor magneton number of the metal. All the ferromagnetic metals were found to yield appreciable $\varepsilon$ values: $\varepsilon\left(\mathrm{Ni} / \mathrm{H}_{2}\right)=-0.37, \varepsilon\left(\mathrm{Cc} / \mathrm{H}_{2}\right)=-0.54, \varepsilon\left(\mathrm{Fe} / \mathrm{H}_{2}\right)=+1.85$. Very few studies have been made with $\mathrm{CO}$ as adsorbate. For adsorbates other than $\mathrm{H}_{2}$ the magnetization studies yield $\zeta$ what is known as bond number $\zeta(\mathrm{x})=\varepsilon(\mathrm{x}) / \varepsilon(\mathrm{H})$, where $\mathrm{x}$ is the adsorbate molecule. Thus the bond number indicates the number of adsorbent atoms involved in the interaction per adsorbate molezule, and one could postulate the possible nature of bonding.

The characteristic magnetic properties; $M_{S}$ (saturation magnetization), $M_{\Gamma}$ (remanent magnetization) and $\mathrm{H}_{\mathrm{C}}$ (coercive field) have been determined using Digital Measurement Systems Vibrating Sample Magnetometer. Figure 3 is a block diagram of the experimental system. This is a Model $880 \mathrm{~A}$ DMS VSM, with an accuracy of $1 \%$, and a sensitivity of 50 micro EMU with one average. The sensitivity can be increased to 5 micro EMU with 100 averages. The system is microprocessor controlled and auto ranges full scale measurement from 0.04 EMU-4000 EMU. In the present arrangement a maximum magnetic field of $13.5 \mathrm{k}$.Oe can be applied and it can be programmed to make temperature dependent measurernents in the range $-192^{\circ} \mathrm{C}$ to $+740^{\circ} \mathrm{C}$. A typical hysteresis curve of a catalyst sample is shown in Figure 4. 
ZFNMR studies along with magnetic measurements have provided an excellent approach in our investigations of the magnetic nature of the inter-metallic interactions in the higher alcohol synthesis copper-cobalt catalyst systems [18-24]. 


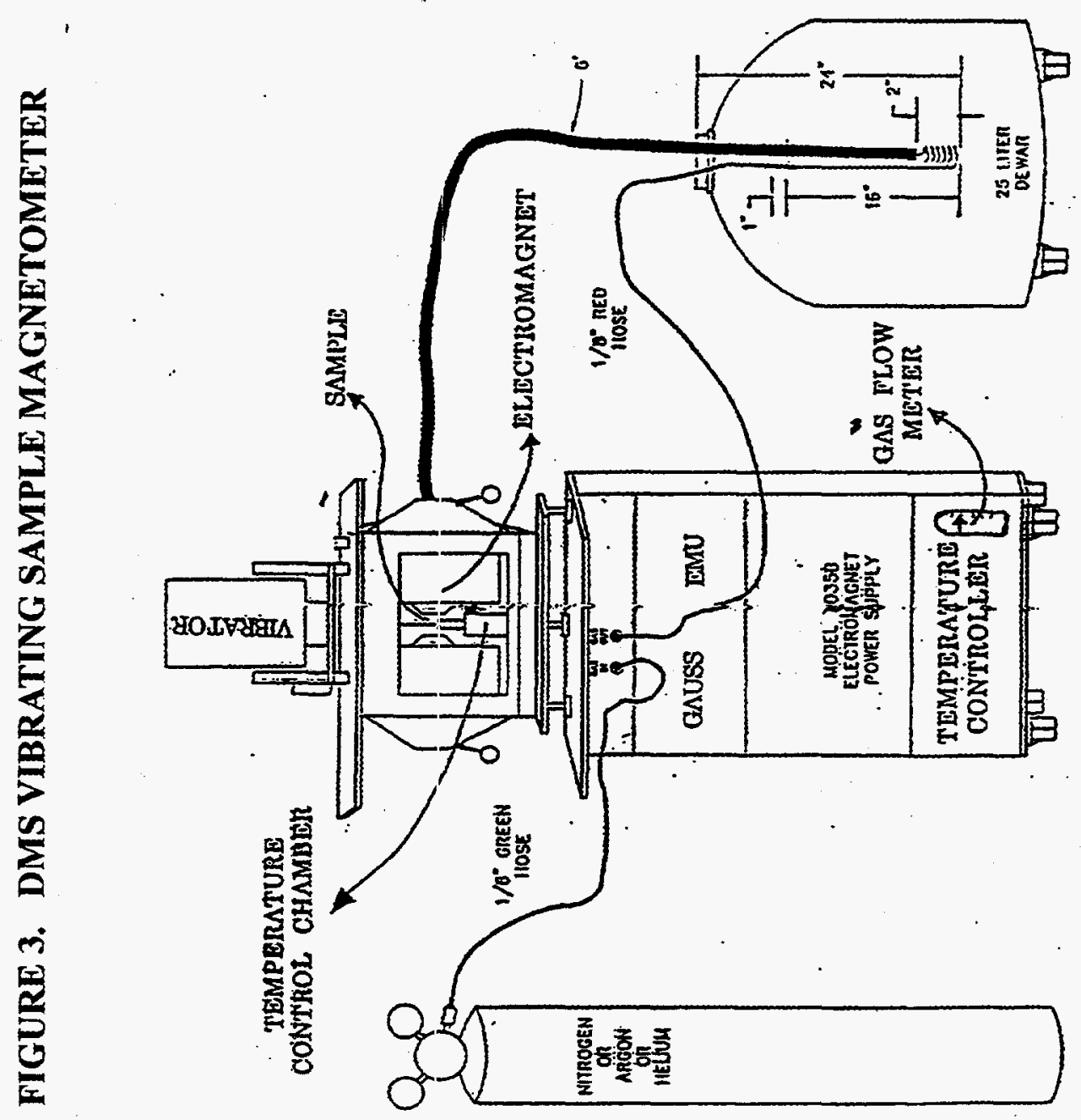




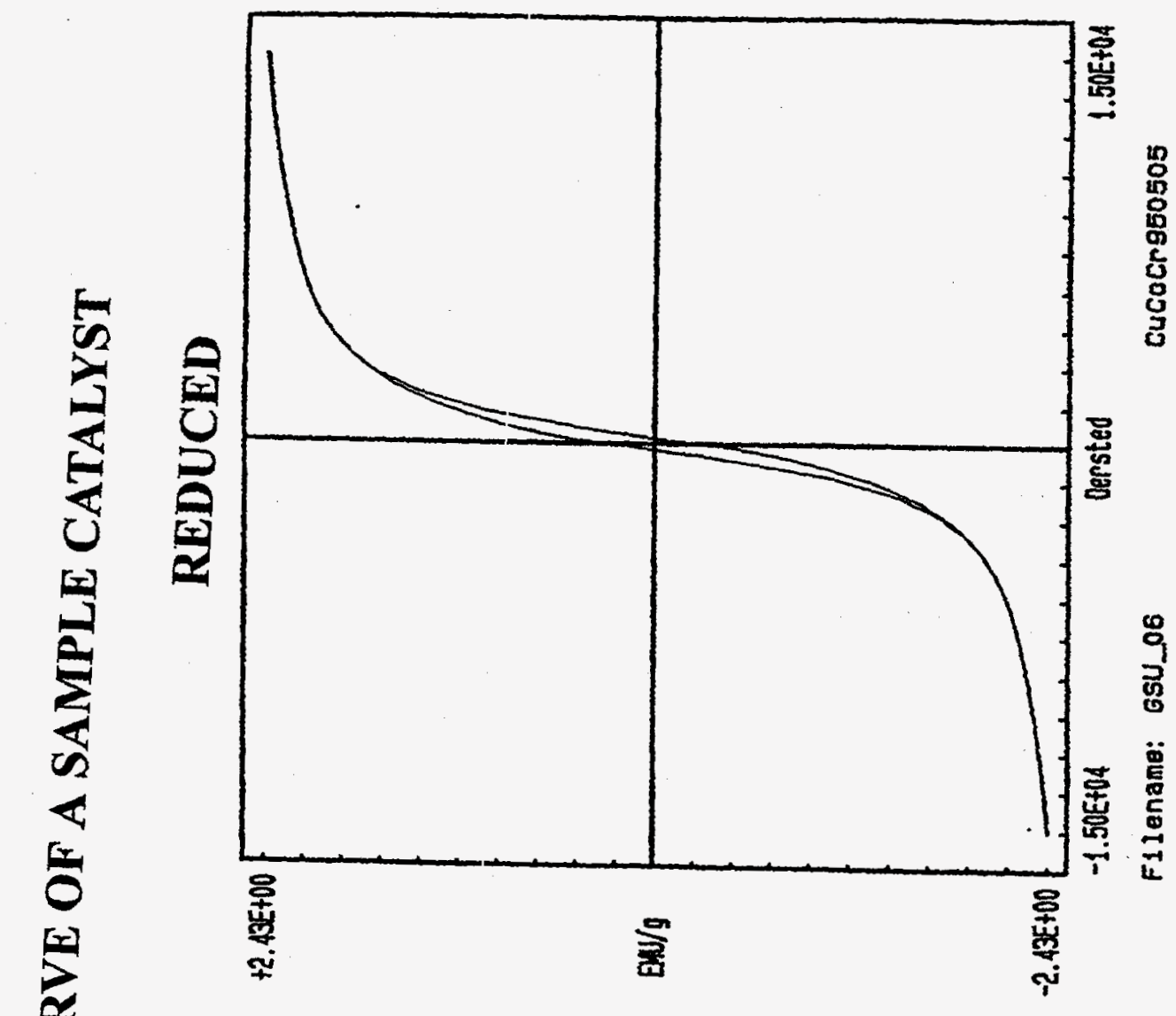

0
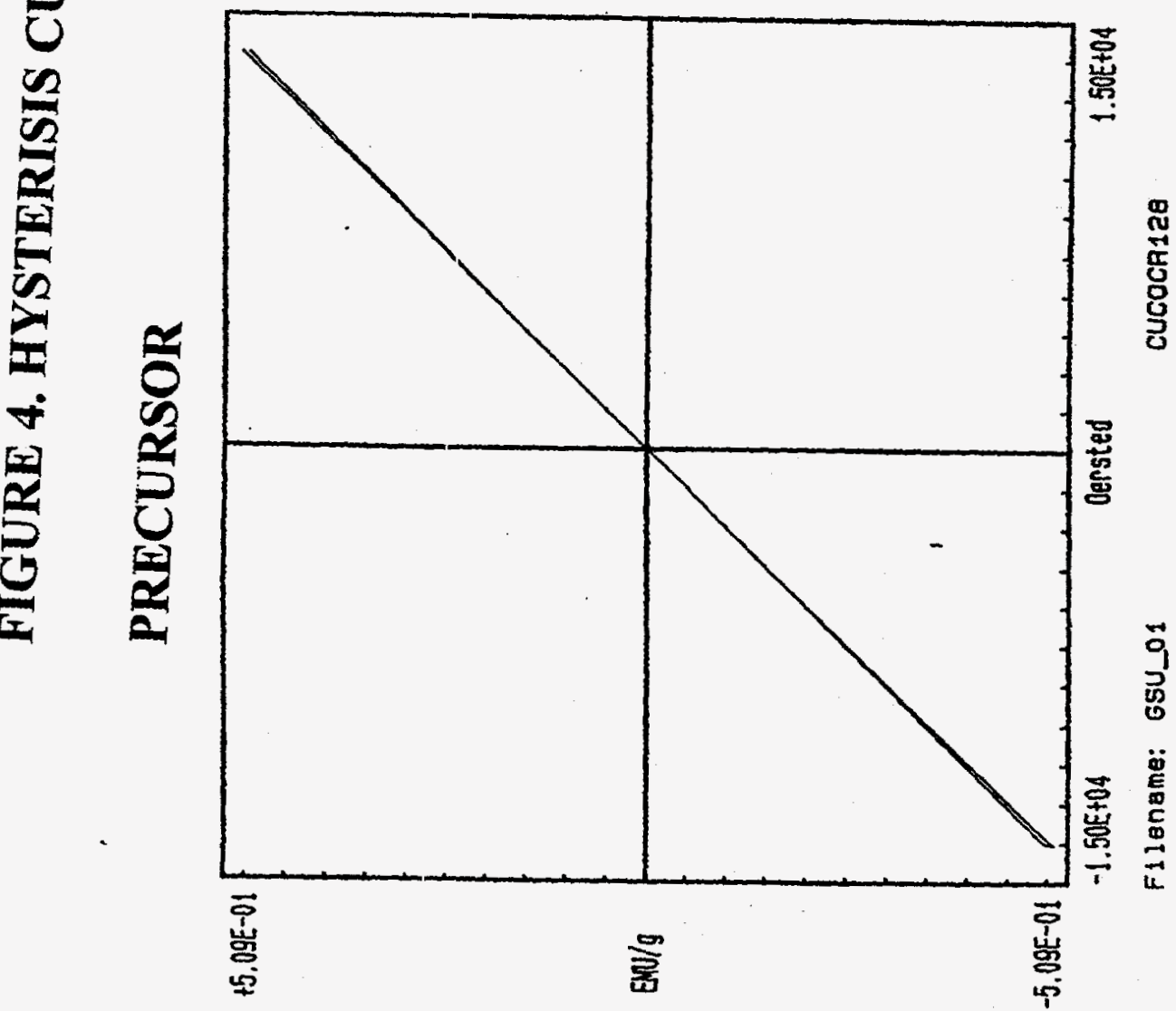


\section{Fourier Transform Infrared Spectroscopy (FTIR)}

Several investigators have successfully employed FTIR and IR spectroscopy techniques for the surface characterization of $\mathrm{Cu} / \mathrm{Co}$ catalysts using $\mathrm{CO}$ as a probe molecule [25-29]. In general, spiectroscopic techniques are found to be most precise in the analysis of vibrational and rotational structures of molecules $[30,31]$.

When the probe molecule is adsorbed on the surface of the catalyst there are two possible electronic interactions between the adsorbent and the adsorbate. One is electron donation (s-electron) from the probe molecule to the empty d-orbital of the metal cation, the effect is strengthening of $\mathrm{C}-\mathrm{O}$ bond of $\mathrm{CO}$. Then $\mathrm{CO}$ vibrational frequency shifts to a higher value. The other interaction is the back- donation of the d-electron from the metal cation to an antibonding orbital of $\mathrm{CO}$. The effect is weakening of the $\mathrm{C}-\mathrm{O}$ bond of $\mathrm{CO}$ and $v_{\mathrm{CO}}$ shifts to a lower frequency. A knowledge of the change in the stretching frequency of $\mathrm{CO}$ reveals the nature and extent of intra and intermolecular interactions in the catalyst. The differences in the frequency shifts from one catalyst to the other which differ either in composition or preparative procedure would shed light on the catalytic character. The FTIR and rnagnetization studies complement each other in the development of a picture of adsorbate and adsorbent interactions and their relation to observed selectivity character.

Several FTIR studies of $\mathrm{CO}$ adsorbed Cu-Co catalysts were performed $[28,29]$ using transmission spectroscopy. This technique seems to have several disadvantages. Most metal catalysts supported on metal oxides are opaque to IR radiation and the use of pressed discs, provides less surface area per gram of adsorbent available to adsorbate molecule. In recent years, Diffise Reflectance Infrared Fourier Transform Spectroscopy 
(DRIFT) has been widely used [32-36] for powdered samples due to a) it's ease in sample handling, b) enhancement in percentage of adsorption, and c) increase in the area of contact of the adsorbent with the gas. In our FTIR investigation of CO adsorption character, we have employed the DRIFT technique.

The experimental set up consists of Mattison Research series FTIR spectrometer, equipped with an MCT detector operable in the mid $I R$ region $\left(4000-600 \mathrm{~cm}^{-1}\right)$, a diffuse reflectance accessory, an environnental chamber and an automatic temperature controller.

A block diagram of the optics of the diffuse reflection attachment is shown in Figure 5. The praying mantis design incorporates two $6: 1,90^{\circ}$ off-axis ellipsoidal mirrors, $M_{3}$ and $M_{4}$, which subtend $20 \%$ of the $2 \pi$ solid angle. These ellipsoids are arranged with a common focal point $S$. Mirrors $M_{1}$ and $M_{2}$ transfer the $\mathbb{R}$ beam from the spectrometer to the first of these ellipsoids $M_{3}$. This ellipsoid focuses the beam onto the sample, $S$. The second ellipsoid $\left(\mathrm{M}_{4}\right)$ collects the radiation diffusely reflected from the sample. This radiation is then directed by mirrors $\mathrm{M}_{5}$ and $\mathrm{M}_{6}$ towards the detector. The environmental chamber (Figure 6), a stainless steel reaction chamber, consists of a sample cup to hold the sample, two windows at the entrance and exit positions for the incident and reflected infrared radiations. A third window is provided at the back of the chamber to illuminate or view the sample. In addition two entry ports are provided for evacuation and gas entry and another two for water circulation. The environmental chamber is also provided with an automatic temperature controller and can be heated up to $600^{\circ} \mathrm{C}$. Spectra can be recorded in the Mid-IR region at resolutions of 1,2 , or $4 \mathrm{~cm}^{-1}$. The noise level can be decreased by increasing the gain and the number of scans. 


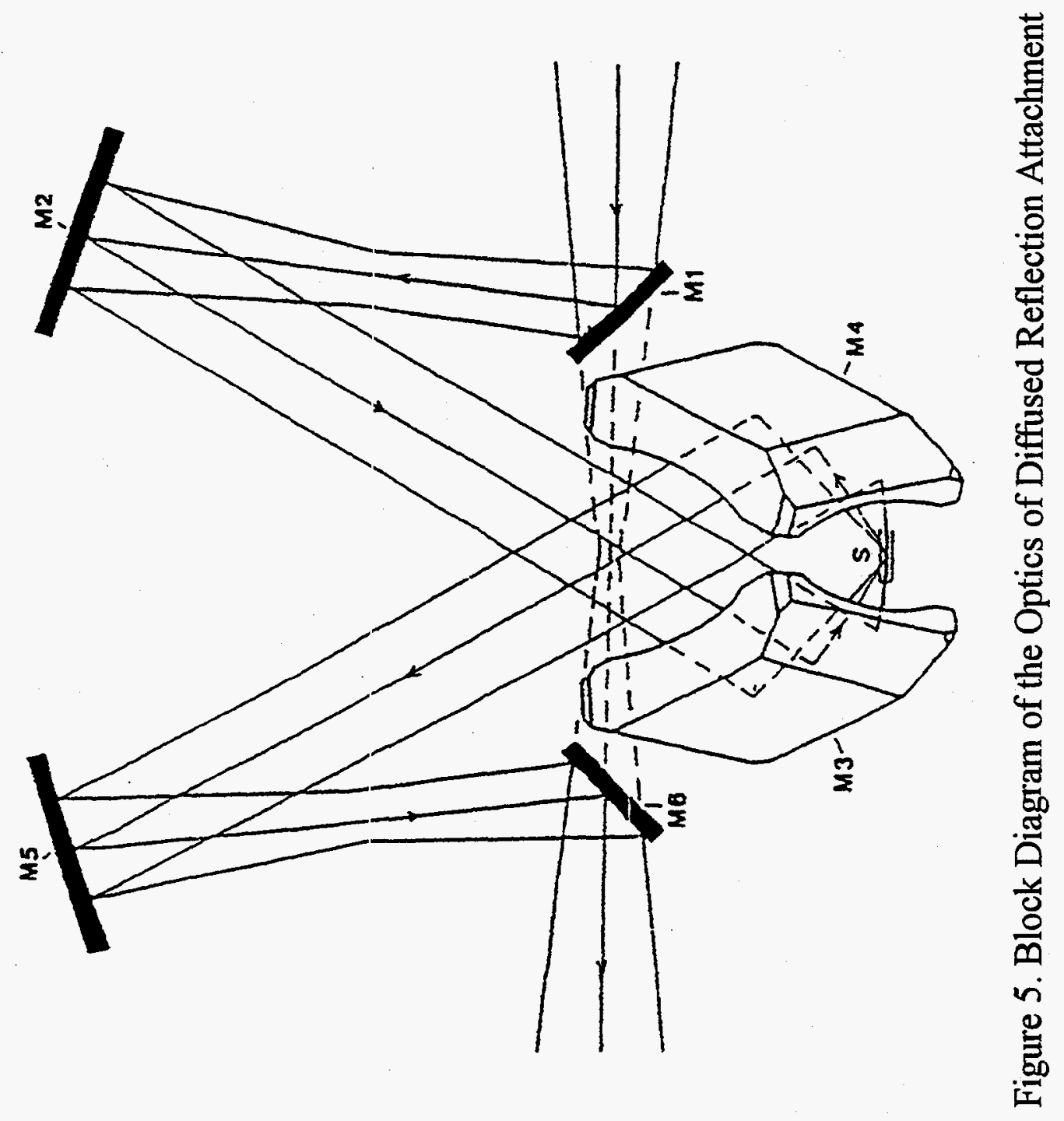




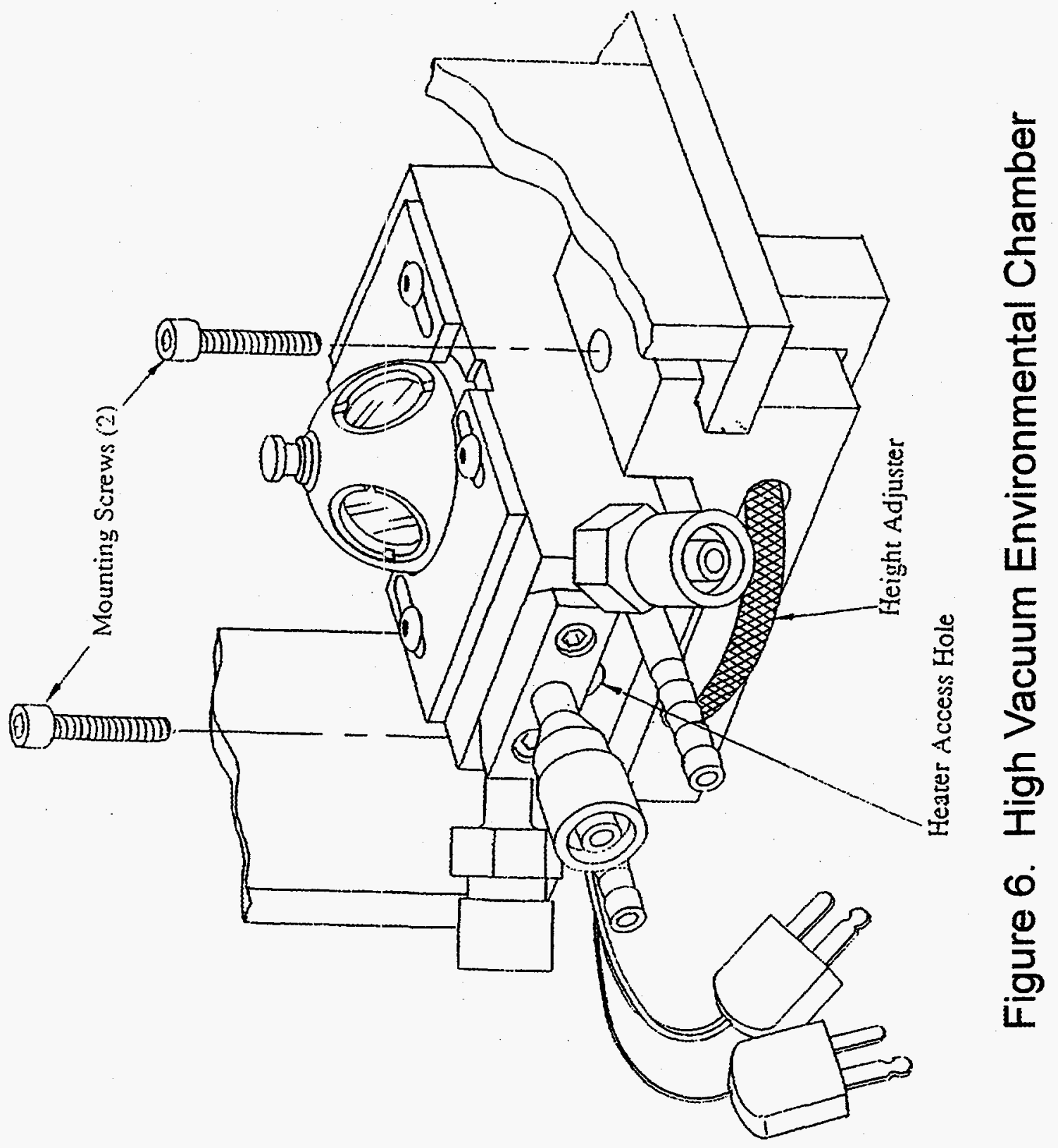




\section{WORK PERFORMED: RESULTS AND DISCUSSION}

\section{A. Sample preparation methodls:}

Since the surface composition and morphological character of the catalyst is sensitive to the method of preparation, we employed three distinct protocols to control the structure of the metal clusters. The methods are very close to those suggested by the earlier investigators. Alerasool and Gonzalez [37] reported that when $\operatorname{Pt}\left(\mathrm{NH}_{3}\right)_{4}\left(\mathrm{NO}_{3}\right)_{2}$ and $\mathrm{Ru}\left(\mathrm{NH}_{3}\right)_{6} \mathrm{Cl}_{3}$ were used as metallic precursors, the structure of the resulting bimetallic catalysts was strongly dependent on the method of preparation. On co-impregnation the surface composition of the resulting bimetallic catalysts was slightly enriched in Pt. In sequential impregnation the order in which the impregnation is carried out was crucial in the resulting structure of the birnetallic particles. When the sequence $\operatorname{Pt}\left(\mathrm{NH}_{3}\right)_{4}\left(\mathrm{NO}_{3}\right)_{2}$ was followed by drying and exposure of reduced $\mathrm{Pt} / \mathrm{SiO}_{2}$ to a solution of $\mathrm{Ru}\left(\mathrm{NH}_{3}\right)_{6} \mathrm{Cl}_{3}$, bimetallic catalysts were not formed. Only mono dispersed $\mathrm{Pt}$ and $\mathrm{Ru}$ particles were observed. However, when the impregnation sequence was reversed bimetallic particles were clearly observed. These bimetallic particles had a surface composition which was enriched in Pt. To investigate the effect of sequence of precipitation, a series of Cu-Co$\mathrm{Cr} / \mathrm{Cu}-\mathrm{Fe}-\mathrm{Zn}$ catalysts were prepared following two protocols (a) and (b).

\section{a.) Co-Precipitation}

One molar aqueous nitrate solutions of the three metals $\mathrm{Cu} / \mathrm{Co} / \mathrm{Cr}$ or $\mathrm{Cu} / \mathrm{Fe} / \mathrm{Zn}$ are mixed in the desired ratios. The mixed nitrate solution is added slowly, drop wise, to a solution of sodium carbonate/hydroxide maintained at a constant temperature of $75-80^{\circ} \mathrm{C}$ and $\mathrm{pH}$ between $7-8$, while stirring continuously. At the end of precipitation the mixture 
is thoroughly washed in warm water to remove the sodium nitrate. The precipitate is air dried at $80^{\circ} \mathrm{C}$ for $18 \mathrm{hrs}$, calcined at $350^{\circ} \mathrm{C}$ for $18 \mathrm{hrs}$ and reduced at $350^{\circ} \mathrm{C}$ for $18 \mathrm{hrs}$ in a flowing stream of hydrogen. These are designated as type A catalysts.

\section{b.) Sequential precipitation}

In this procedure, desired amount of $1 \mathrm{M}$ aqueous solution of copper nitrate and chromium nitrate are mixed and added slowly drop wise to a $7-8 \mathrm{pH}$ sodium carbonate/hydroxide solution at a temperature of $75-80^{\circ} \mathrm{C}$., while continuously stirring.

At the end of precipitation, the washed precipitate is resuspended in a $7-8 \mathrm{pH}$ sodium carbonate/hydroxide at $75-80^{\circ} \mathrm{C}$, and desired amount of cobalt nitrate/iron nitrate solution is precipitated on top. The precipitate is dried, calcined and reduced as in protocol (a). These are designated as type B catalysts - Co atop $\mathrm{Cu}$.

Maintaining identical intermetallic ratios, a third sample is prepared reversing the sequence of precipitation and repeating protocol (b). These are designated as type C catalysts - $\mathrm{Cu}$ atop $\mathrm{Co}$. Since the intermetallic ratios are constant in the three samples, any changes in the syngas interaction character should reflect the changes in the surface composition/intermetallic interaction produced by the preparative technique. Further, since the three protocols could yield a wide variety of possible compositions and structures of metal clusters for $\varepsilon$ given ratio, our characterization studies are expected to provide a comprehensive picture of the syngas interaction character.

\section{Cu/Co Ratio:}

It has been established by Courty, et al $[38,39]$ that the main product obtained in the copper rich field $(\mathrm{Cu} / \mathrm{Co} \geq 5)$ is methanol, while in cobalt rich field hydrocarbons are 
produced $(\mathrm{Cu} / \mathrm{Co} \leq 1)$. Intermediate compositions in the range $1<\mathrm{Cu} / \mathrm{Co}<3$ yield a mixture of light alcohols $\left(\mathrm{C}_{1}-\mathrm{C}_{6}\right)$ with hydrocarbons $\left(\mathrm{C}_{1}-\mathrm{C}_{6}\right)$ as by-products.

Figure 7 is reproduced from the above publication for the purpose of explaining the basis of our choice of the specific ratios. We investigated twelve distinct composition catalysts to cover the three regions of selectivity suggested by the IFP group [38].

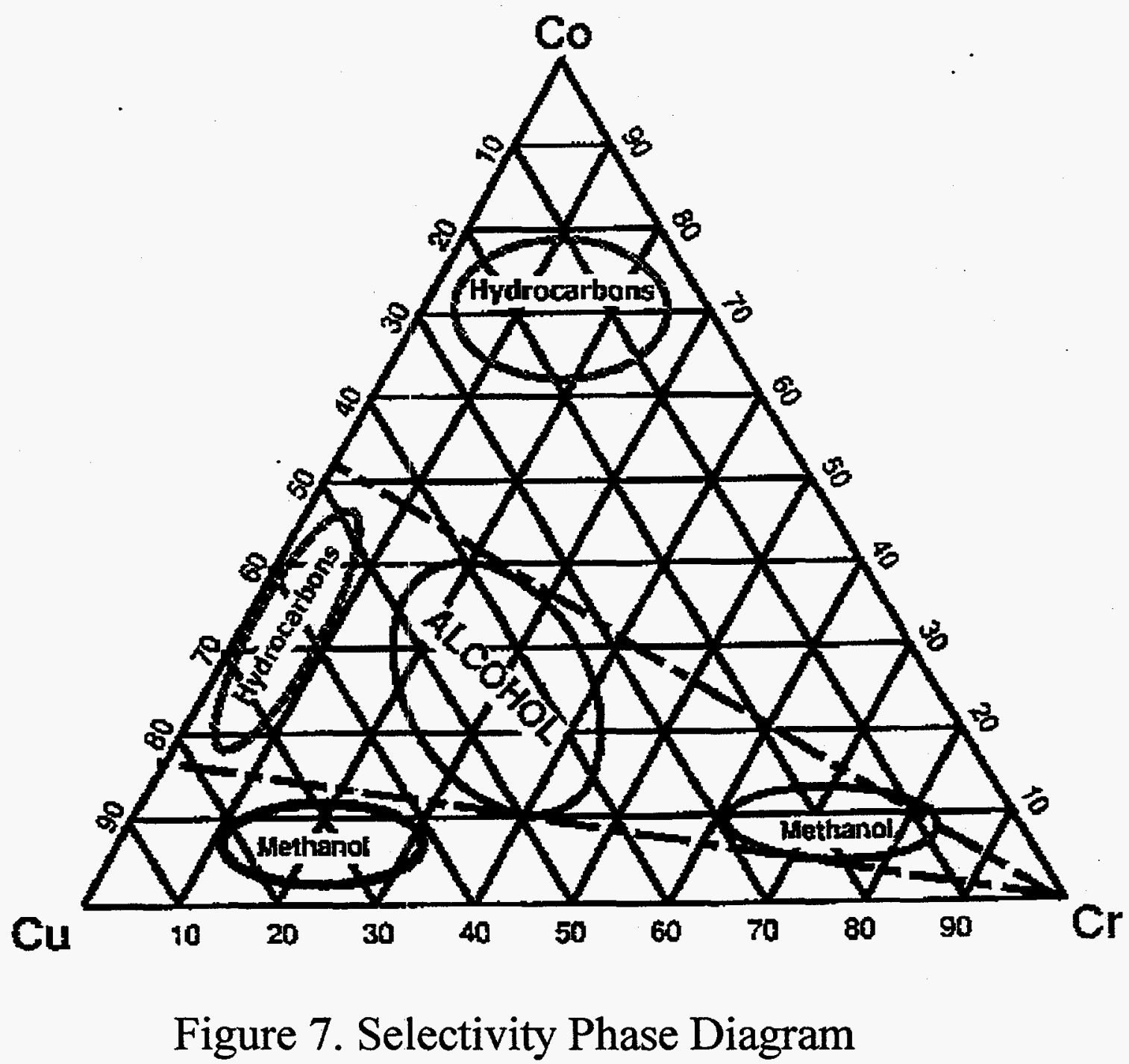


CO adsorption studies were performed using DRIFT technique. Calcined samples are loaded into the sample cup of the DRIFT accessory and evacuated for 1 hour $80^{\circ} \mathrm{c}$. Then hydrogen is passed through the sample for 18 hours at a rate of $20 \mathrm{cc} / \mathrm{min}$ while increasing the temperature to $350^{\circ} \mathrm{C}$. After reduction, hydrogen was pumped out while cooling the sample to room temperature and $\mathrm{CO}$ is admitted at room temperature at the same rate. Spectra are recorded at temperature increments of $50^{\circ} \mathrm{C}$ up to $250^{\circ} \mathrm{C}$. Then CO was desorbed and spectra are recorded in the reverse order while continuing evacuation.

\section{B. Effect of Sequence of Precipitation}

\section{NMR and Magnetization Studies}

A number of catalysts with $\mathrm{Cu} / \mathrm{Co}$ ratio spanning the complete range from 0.1 to 37 were prepared carefully using the co-precipitation (type A) and sequential precipitation (type B and C) techniques described above. Co-precipitation catalysts are designated as Type A, Type $\mathrm{B}$ catalysts are $\mathrm{Co}$ atop $\mathrm{Cu}$ and Type $\mathrm{C}$ catalysts are $\mathrm{Cu}$ atop $\mathrm{Co}$

The NMR results are presented in Figure 8 for three sets of alcohol selective catalysts with pure cobalt spectrum as a background reference (broken vertical lines). The reference spectrum consists of five lines [40]: a line at $213 \mathrm{MHz}$ due to fcc phase particles and a line at $221 \mathrm{MHz}$ due to hcp phase crystallites, and three fault lines in pairs due to twinning and stacking at 215.5 and $218.4 \mathrm{MHz}$ and 215.5 and $217.4 \mathrm{MHz}$. But for minor changes, NMR could not detect any difference in the cobalt spectra for the three types of preparation techniques. This is expected because NMR technique is not sensitive to changes in the surface composition. Interestingly in all the catalysts examined in this series, which are all alcohol selective according to IFP selectivity phase diagram, the hcp 
phase crystallite particles are absent. This leads us to believe fcc phase crystallite particles favor alcohol production. Figure 9 shows the effect of metal ratio on the NMR spectrum of cobalt. As observed earlier [23], chromia inhibits intermetallic interaction and all the spectra are similar, independent of metal ratio, and lie fairly close to the pure cobalt spectrum. However at very high $\mathrm{Cu} / \mathrm{Co}$ ratio we find a couple of weak lines beyond the hcp line which may be due to copper cobalt alloying or formation of solid solution. The absence of significant shifts in NMR lines might suggest that sequence of precipitation does not produce any strong intermetallic interactions. Figure 10 depicts the NMR spectra classified as per the IFP selectivity phase diagram. In the hydrocarbon and alcohol selective catalysts NMR lines are confined to the normally expected cobalt region with minimal scattering indicating that cobalt is essentially structurally unaffected. In the methanol selective regions it seems, as noted earlier, copper cobalt alloying or solid solution formation might be occurring.

The magnetization results showing the effect of sequence of precipitation are presented in Table 1. Comparing the coercive field results for the three preparations of the same metal ratio, co-precipitation samples seem to produce relatively smaller size particles. As the ratio increases, $\mathrm{Cu} / \mathrm{Co}>3$, cobalt seems to go into solid solution with copper and exhibits paramagnetic behavior in all the three preparative protocols. In general $\mathrm{A}$ type (co-precipitation) and $\mathrm{B}$ type ( $\mathrm{Co}$ atop $\mathrm{Cu}$ ) samples, seem to lend themselves for better reduction independent of metal ratio. On the other hand, $\mathrm{C}$ type $(\mathrm{Cu}$ atop $\mathrm{Co}$ ) samples seem to be poorly reduced. When the magnetization data is analyzed according to the IFP selectivity phase diagram (Table 2), we find that all catalysts in the methanol region show paramagnetic behavior consistently. This suggests that the 
ferromagnetic metal has no role in the production of methanol. In the hydrocarbon selective region as copper content increases considerable amount of cobalt seems to be locked in $\mathrm{Cu}$-Co spinel structures $\left(\mathrm{AB}_{2} \mathrm{O}_{4}\right)$. In the alcohol selective region the increase in copper content seems to promote cobalt reduction. Copper and cobalt lying in close proximity appear to retain their normal electronic structural characteristics. Such atomic distributions seem to be favorable for synergetic functioning in the production of alcohols. 
Figure 8

\section{A - Coprecipitation \\ B - Co atop Cu \\ C - Cu atop Co \\ cu-co-cr}

37-37-26 A

43-26-31A

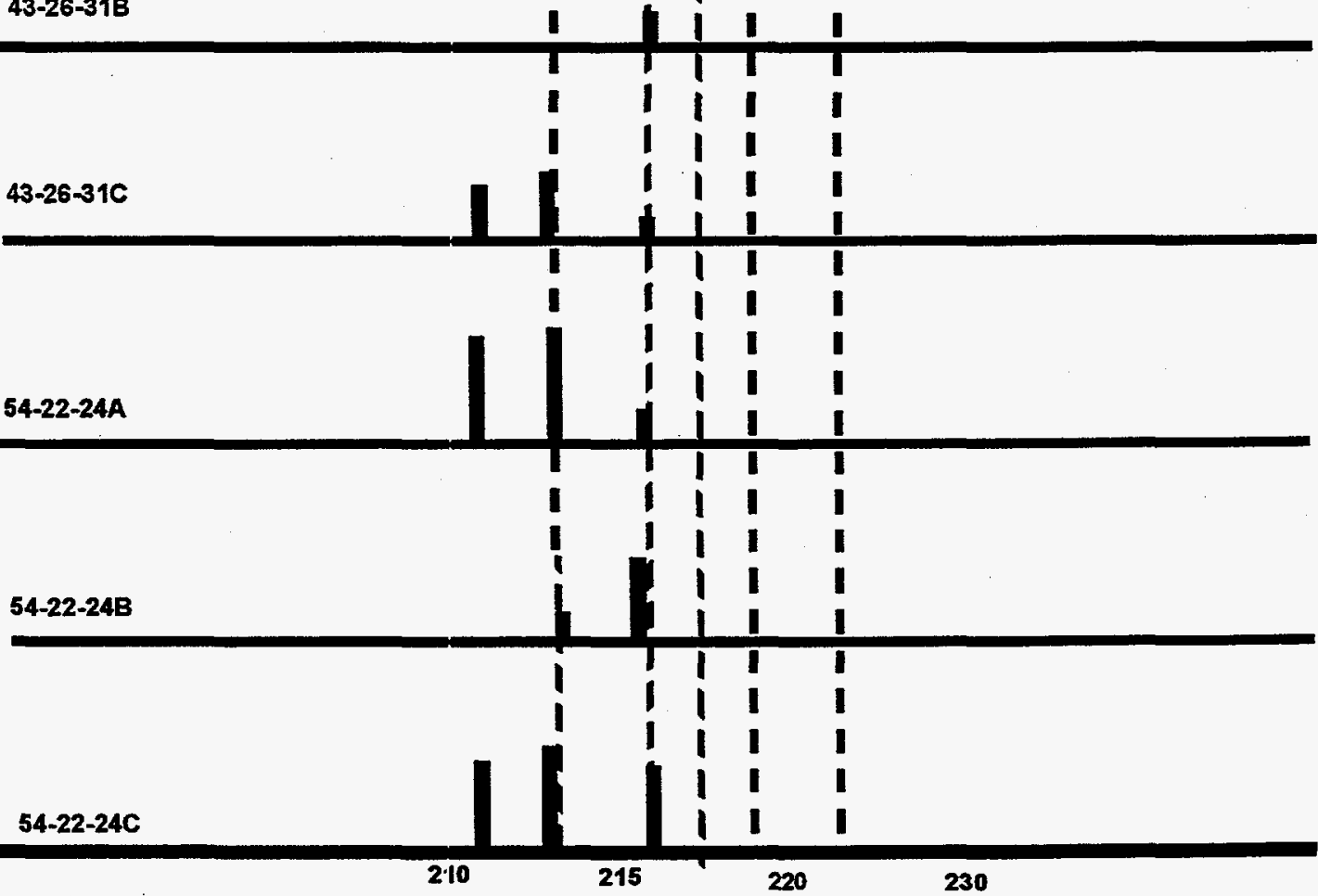

NMR IFrequencies of $\mathrm{CW} / \mathrm{Co} / \mathrm{Cr}$ Catalysts

Alcohols (intermediate range)

\section{EFFE(ST OF METHOD OF PREPARATION}

FCC Fault Lines HCP 
Figure 9 NMR Frequencies of $\mathrm{Cu} / \mathrm{Co} / \mathrm{Cr}$ Catalysts IEFFECT OF METAL RATIO

$\begin{array}{llll}210 & 215 & 220 & 230\end{array}$

CúCo Ratio FCC Fault Lines HCP

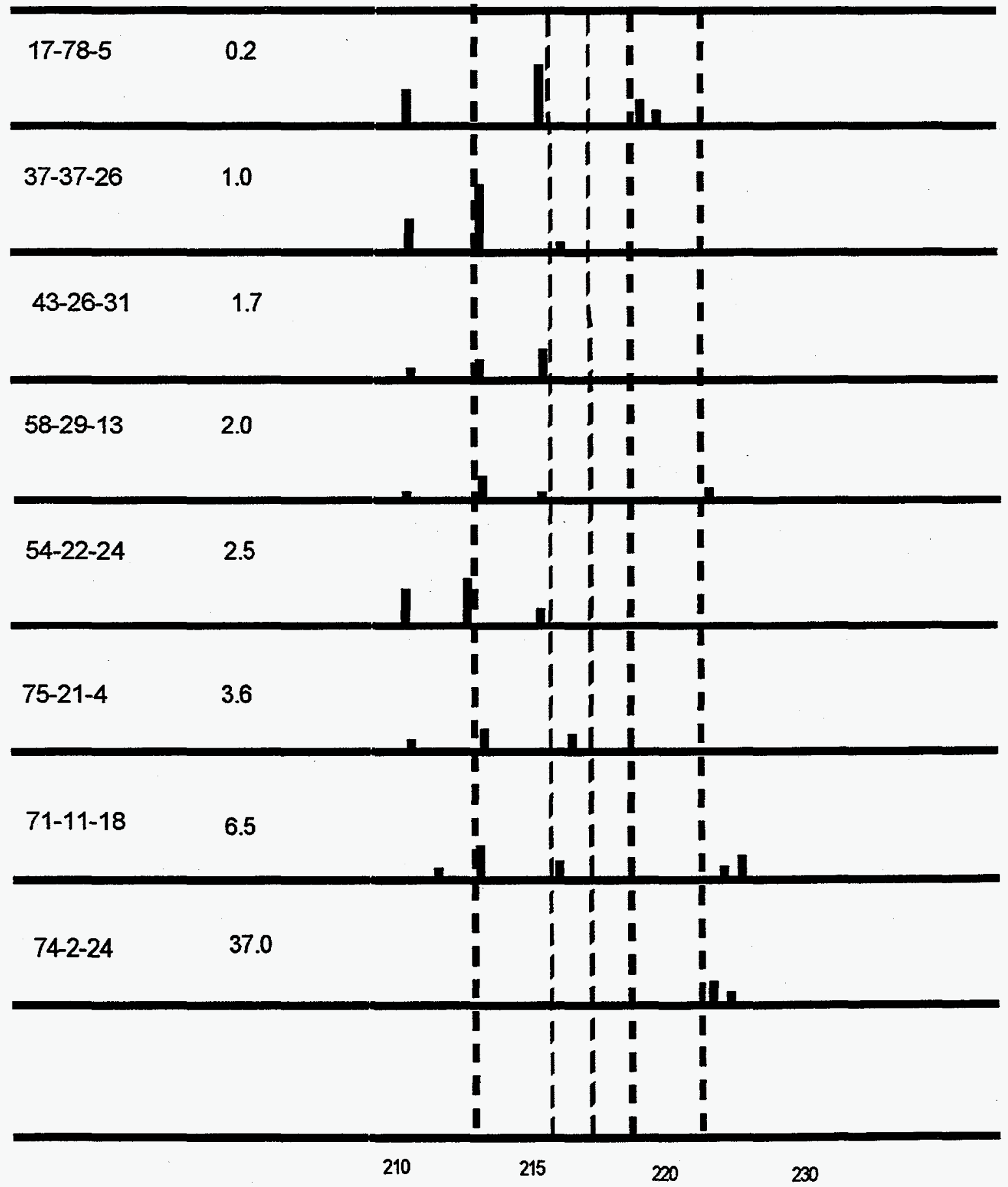


Figure 10

NMR Frequencies of $\mathrm{Cu} / \mathrm{Co} / \mathrm{Cr}$ Catalysts SELECTIVITY CHARACTER

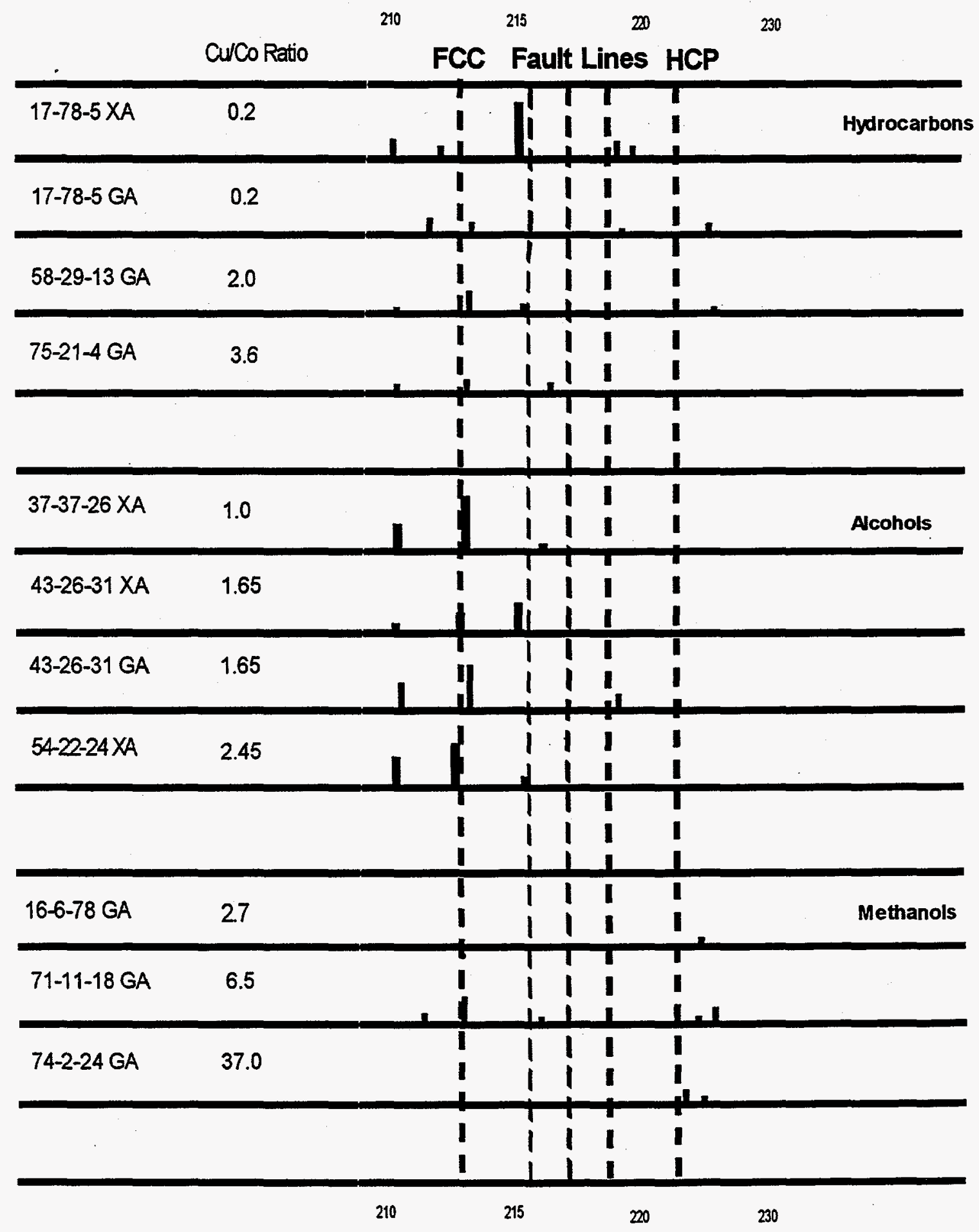


$A=$ Coprecipitation

$\mathrm{B}=\mathrm{Co}$ atop $\mathrm{Cu}$

$\mathrm{C}=\mathrm{Cu}$ atop $\mathrm{Cu}$

Table 1

Effect of sequence of precipitation

\begin{tabular}{|c|c|c|c|c|c|c|}
\hline $\mathrm{Cu} / \mathrm{Co}$ & $\mathrm{Cu} / \mathrm{Co} / \mathrm{Cr}$ & Ss & Hc & Estimated $(\mu)$ & Sr & Sr/Ss \\
\hline Metal Ratio & Composition & $(\mathrm{emu} / \mathrm{g})$ & (Oe) & (emu/g) & $(\mathrm{emu/g})$ & \\
\hline 1 & $37-37-26 \mathrm{~A}$ & 23.5 & 484 & 64 & 78 & 0.33 \\
\hline & $37-37-26 \mathrm{~B}$ & 15.5 & 329 & 42 & 4.2 & 0.03 \\
\hline & $37-37-26 \mathrm{C}$ & 409 & 373 & 11 & 1 & 0.2 \\
\hline & & & & & & \\
\hline 1.7 & $43-26-31 \mathrm{~A}$ & 12.2 & 519 & 47 & 3.9 & 0.32 \\
\hline & 43-26-31 B & 15.5 & 351 & 60 & 4.4 & 0.29 \\
\hline & $43-26-31 \mathrm{C}$ & 12.3 & 433 & 47 & 2.9 & 0.24 \\
\hline & & & & & & \\
\hline 2.5 & 54-22-24 A & 21.1 & 434 & 96 & 4.9 & 0.23 \\
\hline & $54-22-24 \mathrm{~B}$ & 14.4 & 371 & 65 & 4 & 0.28 \\
\hline & $54-22-24 \mathrm{C}$ & 1.4 & 404 & 6 & 0.2 & 0.17 \\
\hline 3.3 & $44-13-43 \mathrm{~A}$ & \multicolumn{5}{|c|}{ PARAMAGNETIC BEHAVIOR } \\
\hline & $44-13-43 B$ & \multicolumn{5}{|c|}{ PARAMAGNETIC BEHAVIOR } \\
\hline & $44-13-43 \mathrm{C}$ & \multicolumn{5}{|c|}{ PARAMAGNETIC BEHAVIOR } \\
\hline
\end{tabular}


Table 2

Selectivity Character of Cu-Co-Cr Catalysts

\begin{tabular}{|c|c|c|c|c|c|c|}
\hline $\begin{array}{l}\text { Cu/Co } \\
\text { Metal Ratio }\end{array}$ & $\begin{array}{l}\mathrm{Cu} / \mathrm{Co} / \mathrm{Cr} \\
\text { Compositio }\end{array}$ & $\begin{array}{l}\text { Ss } \\
\text { (emu/g) }\end{array}$ & $\begin{array}{l}\mathrm{Hc} \\
(\mathrm{Oe})\end{array}$ & $\begin{array}{l}\text { Estimated } \\
\text { (emu/g) }\end{array}$ & $\begin{array}{l}\mathrm{Sr} \\
\text { (emu/g) }\end{array}$ & $\mathrm{Sr} / \mathrm{Ss}$ \\
\hline \multicolumn{7}{|c|}{ HYDROCARBONS } \\
\hline $\begin{array}{c}0.1 \\
0.2 \\
2 \\
3.5\end{array}$ & $\begin{array}{c}8-\bar{\imath} \hat{i}-\bar{\imath} \hat{i} \\
17-78-05 \\
58-29-13 \\
74-2-21\end{array}$ & $\begin{array}{c}01.0 \\
56.5 \\
15.6 \\
5.9\end{array}$ & & $\begin{array}{l}87 \\
72 \\
54 \\
28 \\
\end{array}$ & $\begin{array}{l}14.6 \\
34.2 \\
3.1 \\
2.2 \\
\end{array}$ & $\begin{array}{l}0.23 \\
0.38 \\
0.11\end{array}$ \\
\hline \multicolumn{7}{|c|}{ ALCOHOLS } \\
\hline $\begin{array}{c}1 \\
1.7 \\
2.5 \\
3.4\end{array}$ & $\begin{array}{l}37-37-26 \\
43-26-31 \\
54-22-24 \\
44-13-43\end{array}$ & $\begin{array}{l}23.5 \\
12.2 \\
21.1 \\
\text { P A }\end{array}$ & AMA & $\begin{array}{r}64 \\
47 \\
96 \\
\text { IC BEHA } \\
\end{array}$ & $\begin{array}{l}7.8 \\
3.9 \\
4.9\end{array}$ & $\begin{array}{l}0.33 \\
0.32 \\
0.23\end{array}$ \\
\hline \multicolumn{7}{|c|}{ METHANOLS } \\
\hline $\begin{array}{l}2.7 \\
37 \\
1.8 \\
6.5\end{array}$ & $\begin{array}{l}16-06-78 \\
74-02-24 \\
22-12-66 \\
71-11-18\end{array}$ & \multicolumn{4}{|c|}{$\begin{array}{l}\text { DID NOT REACH SATURATION } \\
\text { PARAMAGNETICBEHAVIOR } \\
\text { DIDNOT REACH SATURATION }\end{array}$} & \\
\hline
\end{tabular}




\section{FTIR Studies:}

Three catalysts with $\mathrm{Cu} / \mathrm{Co}$ ratios $0.2,1$, and 37 , representing the hydrocarbon, alcohol, and methanol selective regions were chosen to examine the effect of sequence of precipitation. Samples were prepared carefully using the co-precipitation (type A) and sequential precipitation (type B and C) techniques described earlier. Each of the calcined sample is loaded in to the sample cup of the Diffuse Reflectance Accessory (DRA), and evacuated for one hour at $80^{\circ} \mathrm{C}$ to remove moisture and adsorbed gases. These were reduced in-situ under hydrogen flow for $18 \mathrm{hrs}$ at $350^{\circ} \mathrm{C}$. Hydrogen was outgassed while decreasing the temperature to room temperature. Carbon monoxide was admitted at: room temperature, and while continuing the CO flow, temperature was increased gradually insteps of $50^{\circ} \mathrm{C}$ and FTIR spectra were recorded at each temperature upto $250^{\circ} \mathrm{C}$. No significant changes were observed in the $\mathrm{C}-\mathrm{O}$ Vibrational frequencies of the spectra taken at different temperatures. FTIR spectra of the three sets of catalysts were graphically represented in Figures 11-13 and the vibrational frequencies are presented in Table 3.

The observed bands can be broadly classified in to five distinct groups [41]. The bands in the region $800-900 \mathrm{~cm}^{-1}$ are due to CO adsorption on chromia , bands in the region 1020-1090 and $1420-1450 \mathrm{~cm}^{-1}$ are due to non coordinated carbonates, bands in the region1040-1080, 13001370 , and $1470-1530 \mathrm{~cm}^{-1}$ are due to Monodentate carbonates, bands in the region $980-1030$, $1220-1270,1530-1670 \mathrm{~cm}^{-1}$ are due to Bidentate carbonates and finally those in the region $1800-$ $2000 \mathrm{~cm}^{-1}$ are due to carbonyls formed by $\mathrm{CO}$ adsorption on cobalt [47].

In the Hydrocarbon selective catalyst $(\mathrm{Cu} / \mathrm{Co}=0.2)$, vibrational frequencies due to carbonyl structures seem to be prornoted in the co-precipitation catalysts, while distinctly absent in the $\mathrm{B}$ and $\mathrm{C}$ catalysts. In alcohol and methanol selective catalysts, these carbonyls structures 
were excited in type B catalysts and were absent in type $\mathrm{A}$ and $\mathrm{C}$ catalysts. As indicated earlier the carbonyl bands are likely associated with $\mathrm{CO}$ adsorbed on positively charged centers of $\mathrm{Co}^{2+}$ ions. The absence of carbonyl bands in type $\mathrm{C}$ catalysts in all the three ratios may be attributed to the non-availability of $\mathrm{Co}^{2+}$ ions on the surface. Also in the type A catalysts ( $\mathrm{Cu} / \mathrm{Co}$ ratios 1.0, 37.0), alcohol and methanol selective catalysts, the surface cobalt atoms available for interaction with $\mathrm{CO}$ may be less due to the percent of cobalt present in the catalyst being low. The basis for the absence of the carbonyl bands in the type B hydrocarbon selective catalyst remains unclear.

Another feature that can be noticed is the absence of bands in the region $1420-1530 \mathrm{~cm}^{-1}$ in type B hydrocarbon selective catalysts and in all type $\mathrm{C}$ catalysts. It has been observed earlier by Nakamoto [48] that frequency values of the coordinated $\mathrm{COO}^{-}$group depend on the metal character. If the degree of ionization is higher, these stretching vibrations may be absent [41]. 
Figure 11: Effect of Sequence of Precipitation $\mathrm{Cu}-\mathrm{Co}-\mathrm{Cr}$ (17-78-5)

$\mathrm{Cu} / \mathrm{Co} / \mathrm{Cr}$ (Hydrocarbon selective)

IIs/III III $\quad \Pi_{a}$ IVA IVB

With Carbon Monoxide

(A) Co-precipitation
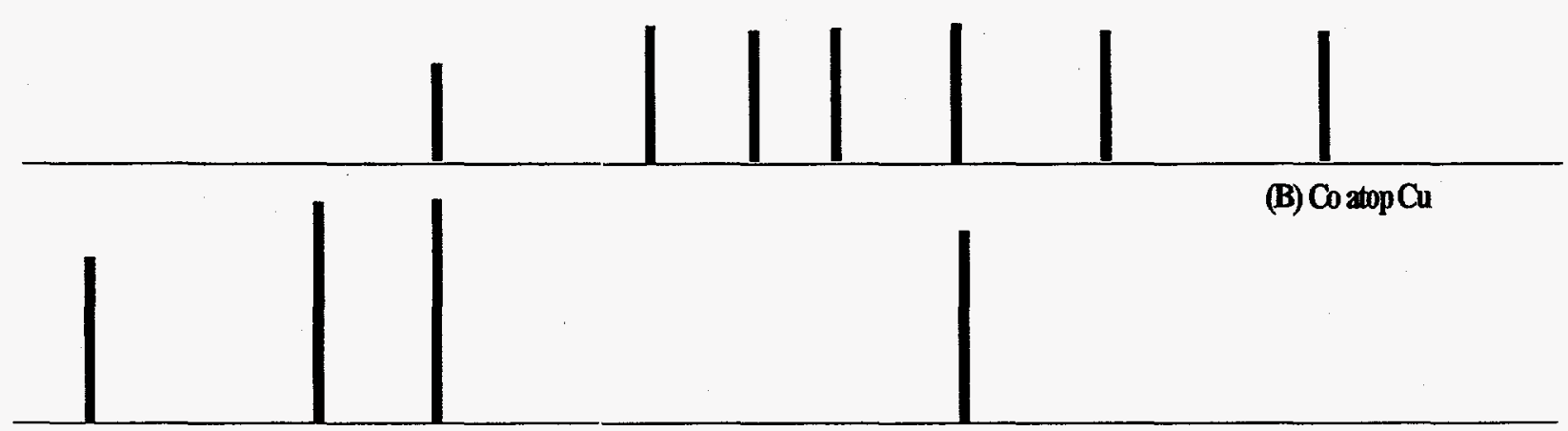

(C) Cu atop $\mathrm{Co}$

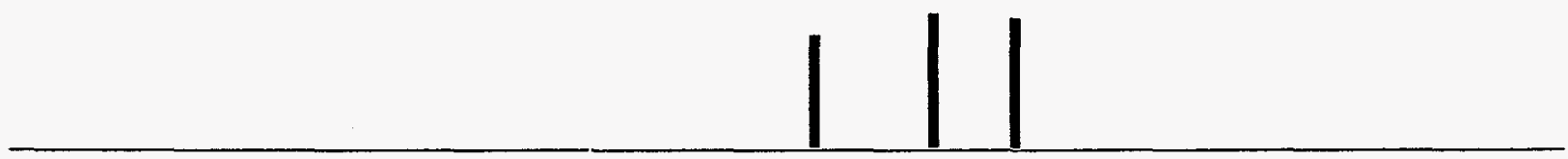

Desorbed

(A) Co-precipitation

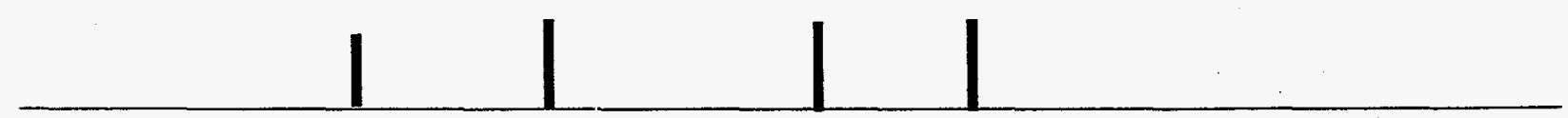

(B) Co atop Cu
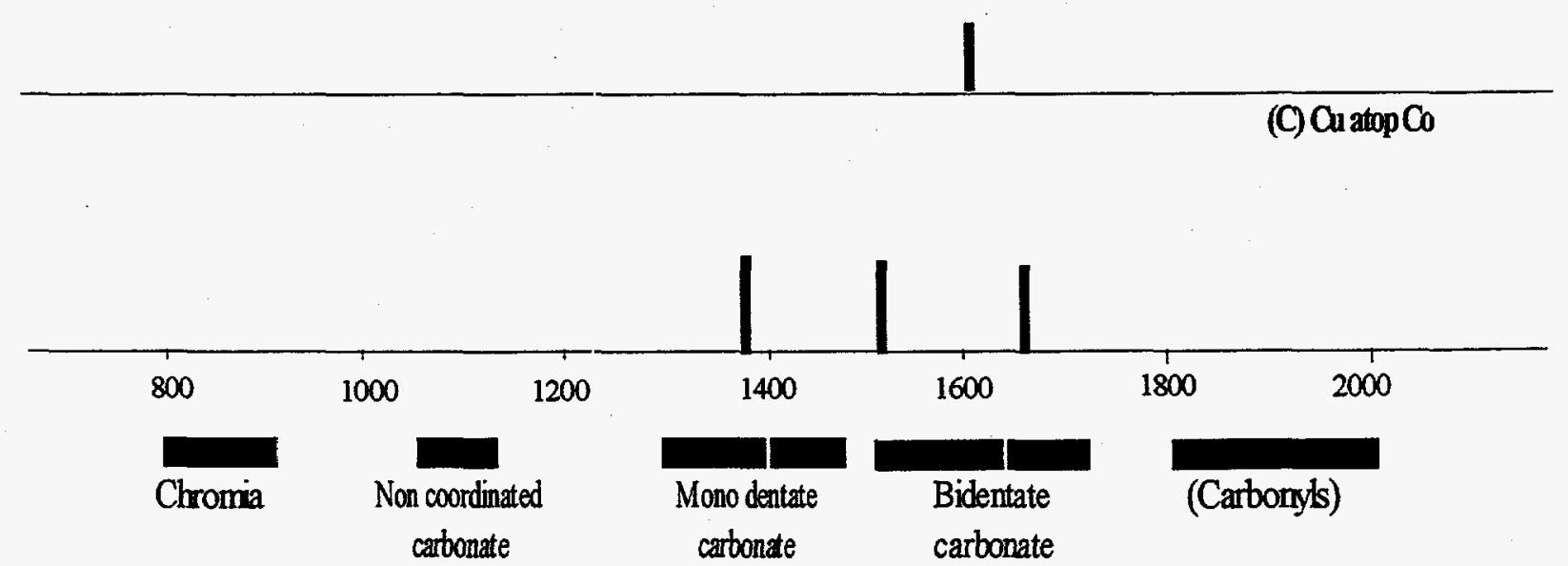


\section{Figure 12: Effect of Sequence of Precipitation $\mathrm{Cu}-\mathrm{Co}-\mathrm{Cr}$ (37-37-2)}

\section{(Alcohol selective)}

$\mathrm{Cu} / \mathrm{Co} / \mathrm{Cr}$

IIS/III III II $\quad$ IVA IVB

$\left(\mathrm{CO}_{4}\right)^{-}$

With Carbon Monoxide

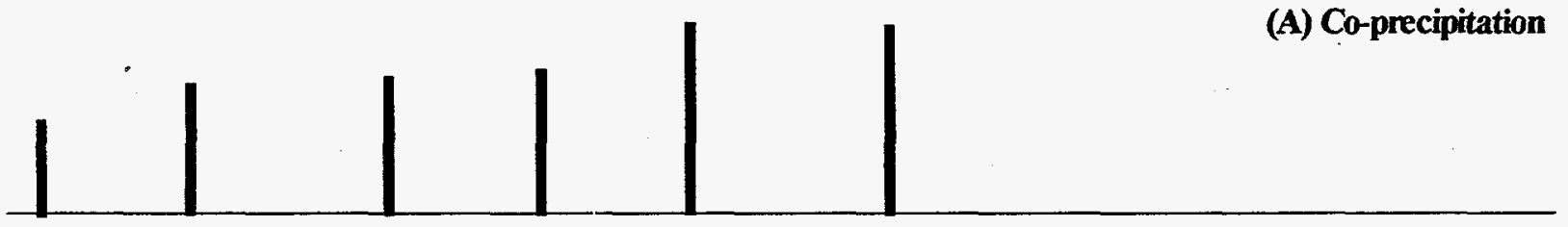

(B) $\mathrm{Co}$ a top Cu

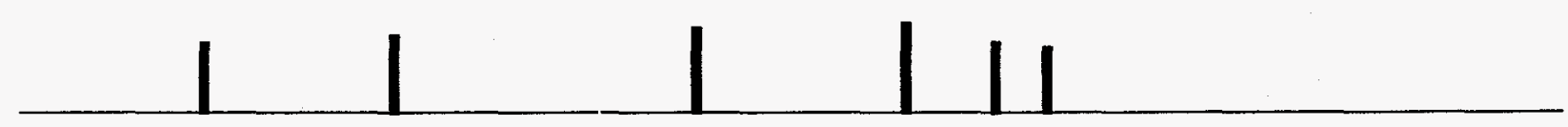

(C) Cu atop Co

$1+1 \quad 1 \quad 11$

Desorbed

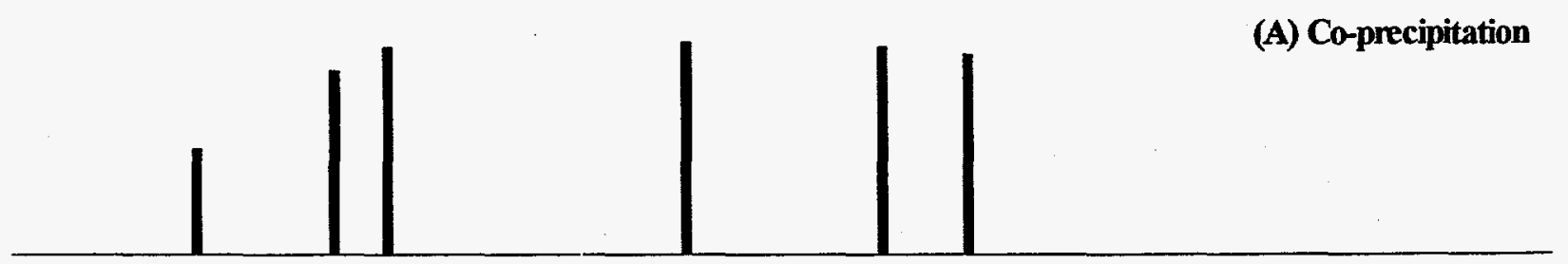

(B) $\mathrm{CoatopCu}$

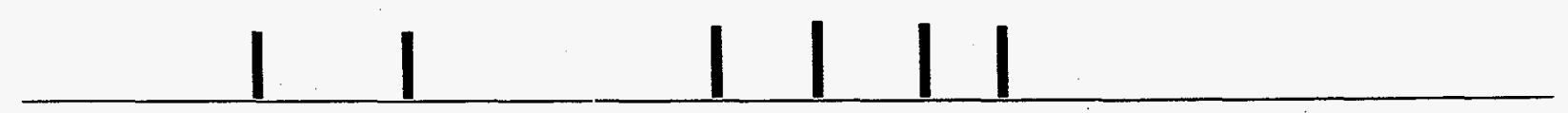

(C) Cuatop Co

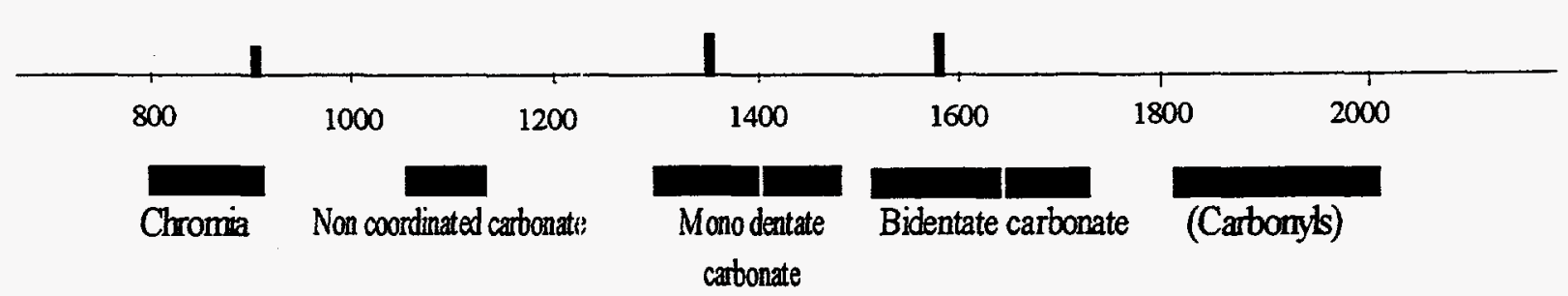




\section{Figure 13: Effect of Sequence of Precipitation Cu-Co-Cr (74-2-24) (Methanol selective) \\ $\mathrm{Cu} / \mathrm{Co} / \mathrm{Cr}$
IIs/III
m
II. IVA IVB
$\left(\mathrm{CO}_{4}\right)^{-}$

With Carbon Monoxide

(A) Co-precipitation

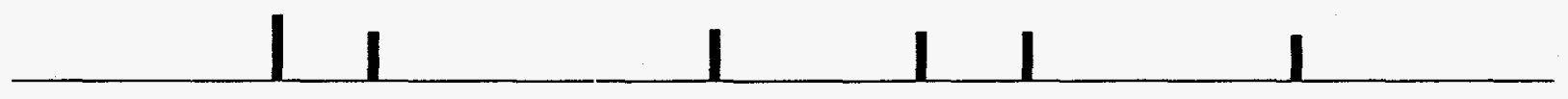

(B) $\mathrm{Co}$ atop $\mathrm{Cu}$
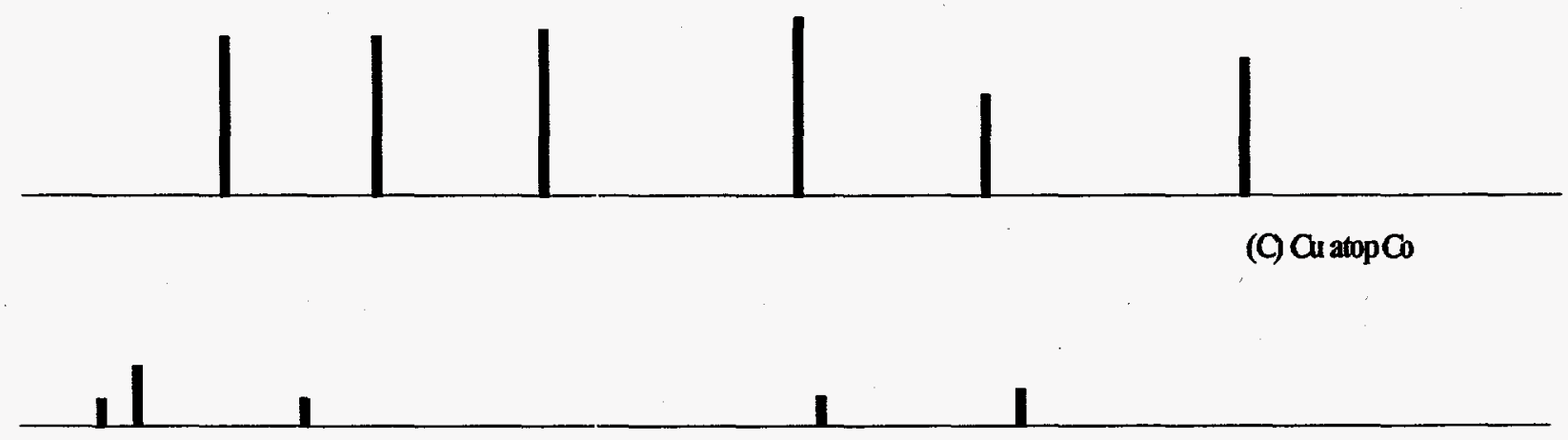

Desorbed

(A) Co-precipitation

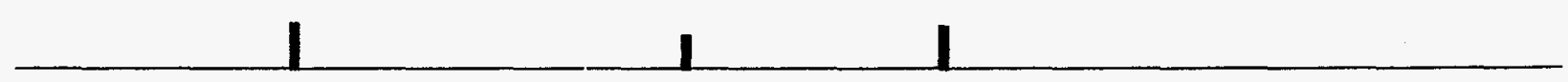

(B) $\mathrm{Co}$ atop Cu

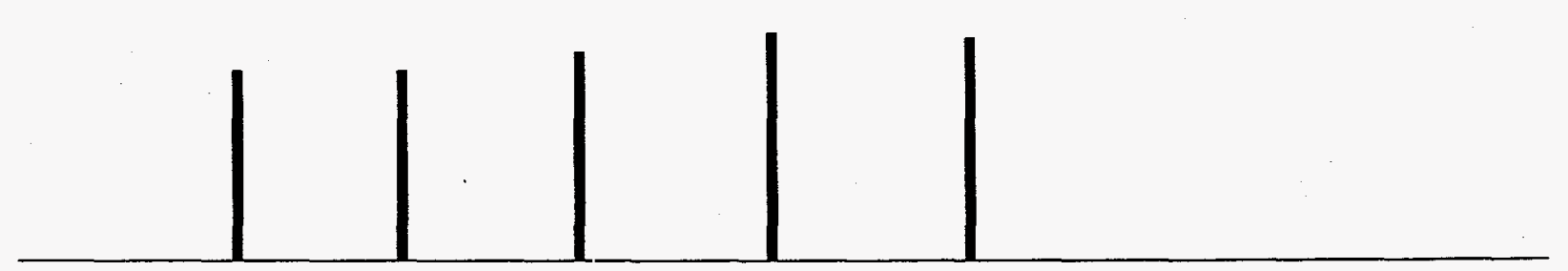

(C) Cu atop Co

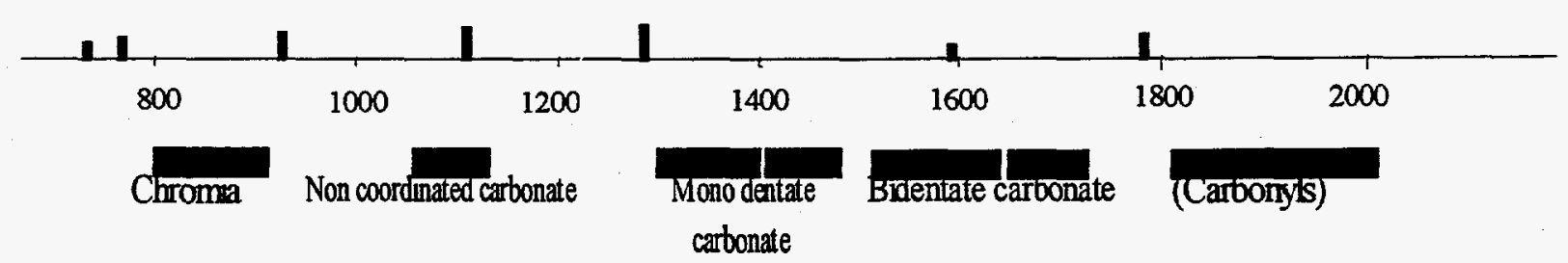


Table 3

Vibrational Frequencies of $\mathrm{Cu}-\mathrm{Co}-\mathrm{Cr}$ Catalysts

(Effect of Sequence of Precipitation)

With Carbon Monoxide

\begin{tabular}{|c|c|c|}
\hline $17-78-5 \mathrm{~A}$ & $17-78-5 \mathrm{~B}$ & $17-78-5 \mathrm{C}$ \\
\hline 1954 & - & - \\
\hline- & - & - \\
\hline 1735 & - & - \\
\hline- & 1600 & 1653 \\
\hline 1585 & - & 1575 \\
\hline 1474 & - & 1468 \\
\hline 1380 & - & - \\
\hline 1276 & - & - \\
\hline- & - & - \\
\hline 1073 & 1074 & - \\
\hline- & 953 & - \\
\hline- & - & - \\
\hline- & 721 & - \\
\hline- & - & - \\
\hline & & \\
\hline
\end{tabular}

\begin{tabular}{|c|c|c|}
\hline $37-37-26 \mathrm{~A}$ & $37-37-26 \mathrm{~B}$ & $37-37-26 \mathrm{C}$ \\
\hline- & - & - \\
\hline- & - & - \\
\hline- & 1693 & - \\
\hline- & 1635 & - \\
\hline 1523 & 1542 & 1566 \\
\hline- & - & 1474 \\
\hline 1326 & 1337 & 1348 \\
\hline- & - & - \\
\hline 1185 & - & - \\
\hline 1022 & 1028 & 1053 \\
\hline- & - & 919 \\
\hline 837 & 842 & 833 \\
\hline- & - & - \\
\hline 691 & - & - \\
\hline & & Bands were very \\
& & woak \\
\hline
\end{tabular}

\begin{tabular}{|c|c|c|}
\hline $74-2-24 \mathrm{~A}$ & $74-2-24 \mathrm{~B}$ & $74-2-24 \mathrm{C}$ \\
\hline 1931 & - & - \\
\hline- & 1883 & - \\
\hline- & - & - \\
\hline 1672 & 1625 & 1667 \\
\hline 1563 & - & - \\
\hline- & 1435 & 1453 \\
\hline 1354 & - & - \\
\hline- & - & - \\
\hline- & 1177 & - \\
\hline 1018 & 1017 & - \\
\hline 923 & - & 944 \\
\hline- & 854 & - \\
\hline- & - & 775 \\
\hline- & - & 727 \\
\hline & & $\begin{array}{c}\text { Bands were } \\
\text { very weak }\end{array}$ \\
\hline
\end{tabular}

\begin{tabular}{|c|c|c|}
\hline- & - & - \\
\hline- & - & - \\
\hline- & - & - \\
\hline 1602 & 1605 & 1663 \\
\hline- & - & 1522 \\
\hline 1450 & - & - \\
\hline- & - & 1381 \\
\hline- & - & - \\
\hline 1178 & - & - \\
\hline- & - & - \\
\hline 989 & - & - \\
\hline- & - & - \\
\hline- & - & - \\
\hline- & - & - \\
\hline & & \\
\hline
\end{tabular}

Desorbed

\begin{tabular}{|c|c|c|}
\hline- & - & - \\
\hline- & - & - \\
\hline- & - & - \\
\hline 1624 & 1649 & - \\
\hline 1542 & 1561 & 1572 \\
\hline- & 1451 & - \\
\hline 1349 & 1350 & 1351 \\
\hline- & - & - \\
\hline- & - & - \\
\hline 1034 & 1041 & - \\
\hline 980 & - & 907 \\
\hline 844 & 896 & - \\
\hline- & - & - \\
\hline- & - & - \\
\hline & & Bands were very \\
\hline & & weak \\
\hline
\end{tabular}

\begin{tabular}{|c|c|c|}
\hline- & - & - \\
\hline- & - & - \\
\hline- & - & 1783 \\
\hline- & 1602 & - \\
\hline 1587 & - & 1592 \\
\hline- & 1408 & - \\
\hline 1326 & - & - \\
\hline- & 1216 & 1278 \\
\hline- & - & 1117 \\
\hline- & 1027 & - \\
\hline 936 & - & 925 \\
\hline- & 868 & - \\
\hline- & - & 773 \\
\hline- & - & 726 \\
\hline $\begin{array}{c}\text { Bands were } \\
\text { very weak }\end{array}$ & & $\begin{array}{c}\text { Bands Were } \\
\text { very weak }\end{array}$ \\
\hline
\end{tabular}




\section{Catalytic Results:}

Three sets of samples were chosen as per the IFP selectivity phase diagram to yield hydro carbons $\mathrm{Cu} / \mathrm{Co}=0.2$, alcohols $\mathrm{Cu} / \mathrm{Co}=1.0$, and methanols $\mathrm{Cu} / \mathrm{Co}=37$. With each metal ratio three samples, co-precipitation (A), $\mathrm{Co}$ atop $\mathrm{Cu}(\mathrm{B})$ and $\mathrm{Cu}$ atop $\mathrm{Co}(\mathrm{C})$ were prepared following the respective protocols discussed earlier. The samples were sent to Dr. F.J. Waller of Air Products Inc., the Industrial partner, for catalytic measurements. The catalytic results are presented in Table 4 . The gratifying feature is, our samples, though not very efficient, follow the product selectivity patterns of IFP $[38,39]$. The disturbing feature is that $\mathrm{CO}_{2}$ formation is relatively high (about $40 \%$ ) and mixed alcohol $\left(\mathrm{C}_{2}-\mathrm{C}_{6}\right)$ yield was maxed only around $10 \%$. Type $\mathrm{C}$ preparation catalysts seems to give maximum ( $85 \%$ ) yield of hydrocarbons among the three. Interestingly the type B catalyst, though falls in the hydrocarbon region of the selectivity phase diagram yielded $18 \% \mathrm{C}_{2}-\mathrm{C}_{6}$ alcohols, highest alcohol yield of all the catalysts prepared. Again the type B catalyst had the maximum methanol yield for $\mathrm{Cu} / \mathrm{Co}=37$. The catalytic results seem to indicate that type $\mathrm{B}$ preparation catalysts, $\mathrm{Co}$ atop $\mathrm{Cu}$ in general performed better with maximum product yield in the specific selertivity regions and the co-precipitation catalysts, type A, performed poorly with very high $\mathrm{CO}_{2}$ conversion.

Table 5 shows the catalytic results on co-precipitated alcohol selective catalysts, $1 \leq \mathrm{Cu} / \mathrm{Co} \leq 3.4$. All the catalysts show poor $\mathrm{CO}$ conversion and an average alcohol production of abut $10 \%$. But for the catalyst $\mathrm{Cu} / \mathrm{Co}=3.4$ which falls in the IFP alcohol selective region, there are no significant differences in the performance. The $\mathrm{Cu} / \mathrm{Co} 3.4$ catalyst exhibit a unique behavior with more than $60 \%$ methanol production. It is also interesting to note that this catalyst exhibits paramagnetic behavior indicating that cobalt 
may be in solid solution with copper. We also find that one can not expect specific selectivity by following rigorously either the ratio rule or the IFP phase diagram. 
Table 4 Catalytic Data Effect of Sequence of Precipitation

\begin{tabular}{|c|c|c|c|c|c|c|}
\hline Sample & $\begin{array}{r}\text { CulCo } \\
\% \\
\end{array}$ & $\begin{array}{r}\mathrm{CO} 2 \\
\% \\
\end{array}$ & \multicolumn{4}{|c|}{ Methanols Mixexd Alcohol Hydrocarbons Unknown } \\
\hline $17-78-5 A$ & & 41 & 1 & 6 & 50 & 2 \\
\hline $17-78-5 B$ & 0.1 & 36 & 3 & 18 & 37 & 6 \\
\hline $17-78-5 \mathrm{C}$ & & 9 & 1 & 1 & 85 & 5 \\
\hline $37-37-26 A$ & & 55 & 5 & 10 & 25 & 5 \\
\hline $37-37-26 B$ & 1 & 20 & 3 & 11 & 62 & 5 \\
\hline $37-37-26 C$ & & 61 & 3 & 6 & 30 & $<1$ \\
\hline $74-2-24 A$ & & 51 & 34 & 2 & 13 & $<1$ \\
\hline $74-2-24 B$ & 37 & 28 & 60 & 4 & 7 & 1 \\
\hline $74-2-24 C$ & & 34 & 52 & 3 & 10 & 1 \\
\hline
\end{tabular}

Table 5

Conversion and Molar Selectivity of Alcohol Selective Catalysts

\begin{tabular}{|cccccccc|}
\hline $\begin{array}{c}\text { Sample } \\
\text { ID }\end{array}$ & $\begin{array}{c}\text { Cu/Co } \\
\text { Metal ratio }\end{array}$ & $\begin{array}{c}\text { CO conv. } \\
\%\end{array}$ & $\begin{array}{c}\text { CO2 } \\
\%\end{array}$ & $\begin{array}{c}\text { Methanols } \\
\%\end{array}$ & $\begin{array}{c}\text { Mixexd Alcohols } \\
\%\end{array}$ & $\begin{array}{c}\text { Hydrocarbons } \\
\%\end{array}$ & $\begin{array}{c}\text { Unknown } \\
\%\end{array}$ \\
\hline $37-37-26$ & 1 & 5 & 55 & 5 & 10 & 25 & 52 \\
$43-26-31$ & 1.7 & 3 & 56 & 3 & 7 & 23 & 2 \\
$54-22-24$ & 2.5 & 10 & 57 & 5 & 11 & 2 & 4 \\
$44-13-43$ & 3.4 & 8 & 27 & 62 & 2 & 8 & 1 \\
\hline
\end{tabular}




\section{Syngas Interaction Studies}

\section{NMR Results and Magnetization Data}

To examine the effect of syngas adsorption on the catalysts, a series of $\mathrm{Cu} / \mathrm{Co} / \mathrm{Cr}$ and $\mathrm{Cu} / \mathrm{Fe} / \mathrm{Zn}$ samples were prepared following protocol (a). After reduction $\mathrm{H}_{2}$ was outgassed while reducing the temperature to $250^{\circ} \mathrm{C}$. At this temperature $\mathrm{CO}$ was admitted for thirty minutes and while continuously flowing $\mathrm{CO}$ the temperature was reduced to room temperature and the samples were sealed under negative pressure. The NMR spectra of Co containing samples are presented in Figure 14.

NMR lines of the hydrocarbon selective catalysts $(\mathrm{Cu} / \mathrm{Co}<1)$ are confined to the normally expected cobalt region (212-221 MHz) with minimal scattering. This suggests that cobalt essentially remains structurally unaffected due to the presence or absence of $\mathrm{CO}$ and that the gas-metal interaction is relatively weak and the absorption process could be more physical in nature. However, as the copper content increases beyond $50 \%$, we find new lines occurring consistently at higher frequencies when $\mathrm{CO}$ is adsorbed on the catalyst. This indicates that the gas-metal interaction (CO-Co) leads to significant modifications in the cobalt $4 \mathrm{~s}-3 \mathrm{~d}$ charge distribution. These catalysts are reported to be alcohol and methanol selective by IFP [38]. The lines persist even after desorption of CO. It seems that the chemisorption process predominates in these catalysts. It appears that strong gas-metal interaction promotes alcohols/methanol production while weak interactions are conducive for hydrocarbon production. 
FIGURE 11 NMR FREQUENCIES OF CU/CO CATALYST Effect of CO Adsorption

$\begin{array}{llll}210 & 215 & 220 & 225\end{array}$

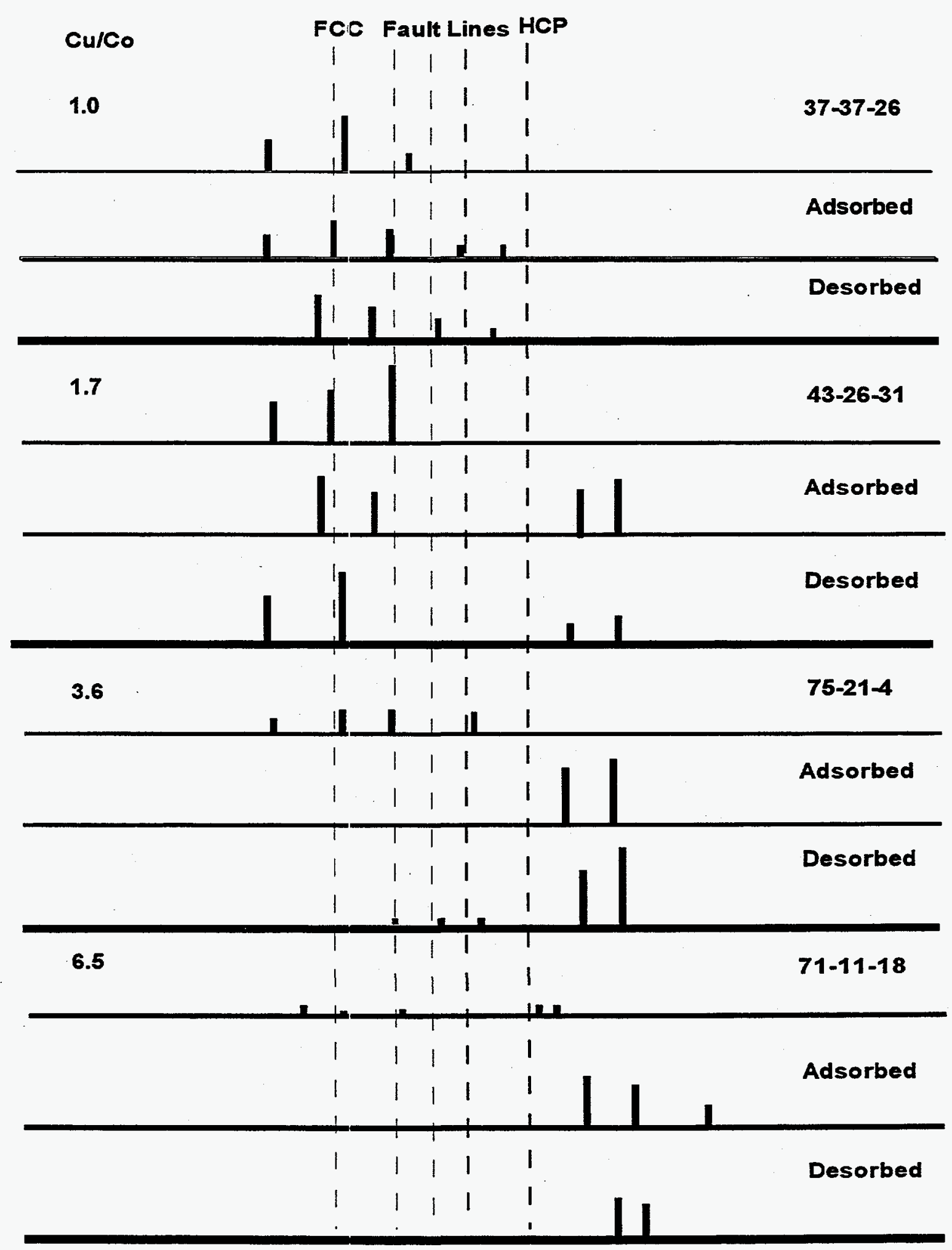


The effect of $\mathrm{CO}$ adsorption on the saturation magnetization of the catalysts is presented in Tables $6(\mathrm{Cu} / \mathrm{Co} / \mathrm{Cr})$ and $7(\mathrm{Cu} / \mathrm{Fe} / \mathrm{Zn})$. An examination of the magnetic moments of the three ferromagnetic metals and their $3 d$ electronic structures: $\mathrm{Fe}\left(3 \mathrm{~d}^{6}\right.$ $218 \mathrm{emu} / \mathrm{g})$, Co $\left(3 \mathrm{~d}^{7}-161 \mathrm{emu} / \mathrm{g}\right)$ and $\mathrm{Ni}\left(3 \mathrm{~d}^{8}-54 \mathrm{emu} / \mathrm{g}\right)$ indicates that the magnetic moment decreases as the $3 \mathrm{~d}$ electron density increases. For all catalysts with $\mathrm{Cu} / \mathrm{Co}<2$ (Table 6), the magnetic moment of the $\mathrm{CO}$ adsorbed catalyst is lower than that for the catalyst. This suggests that there could be charge transfer from carbon monoxide to cobalt and this might be conducive for the production of hydrocarbons. However, as the copper content increases $(\mathrm{Cu} / \mathrm{Co}>2)$, alcohol and methanol selective catalysts, the magnetic moment of the $\mathrm{CO}$ adsorbed catalyst is higher than that for the catalyst. This indicates a reverse charge transfer from cobalt to carbon monoxide. This observation shows a clear link between the selectivity and magnetic character and the nature of syngas interaction with the catalyst.

In all the iron samples analyzed (Table 7), the magnetic moment of the $\mathrm{CO}$ adsorbed catalyst is significantly less than that for the catalyst. Again, this is indicative of charge transfer from carbon monoxide to iron. Unlike $\mathrm{Cu} / \mathrm{Co} / \mathrm{Cr}$ catalysts, this feature is independent of $\mathrm{Cu} / \mathrm{Fe}$ ratio. This leads us to believe that all the $\mathrm{Cu} / \mathrm{Fe} / \mathrm{Zn}$ catalysts studied are likely to be hydrocarbon selective.

The last column in both tables presents the magnetization data of the $\mathrm{CO}$ desorbed samples. Desorption of CO was carried out by simply outgassing the samples over night for nearly 18 hours at room temperature. Both Co and Fe catalysts, consistently show a significant further drop in the magnetic moment. This further drop in the magnetic moment may be indicative of the possibility of enhancement in metal carbide formation. 
TABLE 6

Magnetization data ON $\mathrm{Cu} / \mathrm{Co} / \mathrm{Cr}$ Catalysts

\begin{tabular}{ccccc}
\hline & \multicolumn{4}{c}{ SIGMA (emu/gCo) } \\
\hline SAMPLE ID & Cu/Co & Cu/Co/Cr & Cu/Co/Cr+CO & $\begin{array}{c}\text { Cu/Co/Cr+CO } \\
\text { DESORBED }\end{array}$ \\
\hline $8-71-21$ & 0.11 & 87.2 & 69.9 & 64.10 \\
$17-78-5$ & 0.22 & 117.0 & 83.6 & 37.10 \\
$37-37-26$ & 1.00 & 17.0 & 14.2 & 12.20 \\
$58-29-13$ & 2.00 & 30.7 & 33.4 & 28.90 \\
$54-22-24$ & 2.50 & 6.0 & 19.6 & 9.91 \\
$74-21-5$ & 3.50 & 28.3 & 71.9 & 50.76 \\
$71-11-18$ & 6.50 & \multicolumn{5}{c}{ DID NOT REACH SATURATION } \\
\hline
\end{tabular}

TABLE 7

Magnetization data ON Cu/Fe/Zn Catalysts

\begin{tabular}{ccccc}
\hline & & \multicolumn{3}{c}{ SIGMA (emu/gFe) } \\
\hline SAMPLEID & Cu/Fe & Cu/Fe/Zn & Cu/Fe/Zn+CO & $\begin{array}{c}\text { Cu/Fe/Zn+CO } \\
\text { DESORBED }\end{array}$ \\
\hline $8-71-21$ & 0.11 & 223.4 & 83.5 & 42.18 \\
$17-78-5$ & 0.22 & 164.6 & 82.9 & 79.09 \\
$37-37-26$ & 1.00 & 154.6 & 78.9 & 40.48 \\
$58-29-13$ & 2.00 & 135.5 & 66.2 & 26.19 \\
$54-22-24$ & 2.50 & 142.7 & 64.5 & 19.87 \\
\hline
\end{tabular}


In summary, this study clearly establishes that changes in selectivity character of the catalyst are associated with corresponding changes in the magnetic character and that the nature of the charge transfer between the adsorbate and adsorbent influences both the catalytic and magnetic properties. At low copper concentrations (hydrocarbon selective catalysts), though $\mathrm{CO}$ adsorption does not seem to produce significant changes in the hyperfine field at Co nuclear site, there occurs a drop in the magnetic moment of the catalyst. As the copper content: increases beyond 50\%, (alcohol and methanol selective catalysts), the hyperfine field significantly increases at the cobalt nuclear site and the magnetic moment of the catalyst also increases. In iron catalysts there is a consistent drop in the magnetic moment and seems to be independent of $\mathrm{Cu} / \mathrm{Fe}$ metal ratio.

A series of a fresh batch of catalysts with $\mathrm{Cu} / \mathrm{Co}$ ratio varying from 0.5 to 4.5 were prepared using co-precipitation technique. The catalysts were exposed to carbon monoxide, and a set of three typical catalysts with $\mathrm{Cu} / \mathrm{Co}=0.2,1$, and 37 were exposed to Syngas $\left(\mathrm{CO}+\mathrm{H}_{2}\right)$ and the NMR spectra were scanned in the frequency region 210 $230 \mathrm{MHz}$. We expected that if there occurs any electronic charge transfer between cobalt and carbon monoxide, the hype.fine field at the Co nuclear site will change producing a shift in the NMR spectral lines of cobalt. Positive and negative frequency shifts would indicate the direction of charge transfer between $\mathrm{Co}$ and $\mathrm{CO}$.

The NMR data presented in Table 8 shows that in most of the catalysts prepared, nice crystallites were formed writh no noticeable fault structures. The heat treatment procedure during precipitation, drying, calcination or reduction seems to have lead to the segregation of chromia, promoting copper-cobalt inter-metallic interaction. Generally NMR lines are shifted to higher frequencies in most of the samples. The presence or 
absence of carbon monoxide maide no difference to the NMR results. The second set of three samples exposed to Syngas neither show any unique features attributable to Syngas adsorption. The morphological structure of the samples seems to mask the subtle effects due to $\mathrm{CO}$ or $\mathrm{CO}+\mathrm{H}_{2}$ adsorption.

Magnetization properties of $\mathrm{Cu} / \mathrm{Co} / \mathrm{Cr}$ catalysts, prepared following coprecipitation technique, were investigated using a Digital Measurement Systems (DMS)Vibrating Sample Magnetometer (VSM) at GSU. Several sets of samples with different $\mathrm{Cu}, \mathrm{Co}, \mathrm{Cr}$ elemental compositions were examined. The magnetization studies on all the samples were made at room temperature after different treatments such as: after reduction, after adsorption of $\mathrm{CO}$, after desorption of $\mathrm{CO}$, and after adsorption and desorption of Syngas $(\mathrm{CO}+\mathrm{H} 2)$. The complete data is presented in Table 9. The magnetization data is analyzed separately for hydrocarbon selective catalysts $(\mathrm{Cu} / \mathrm{Co}<1.5)$ and alcoholmethanol selective catalysts $(\mathrm{Cu} / \mathrm{Co}>1.5)$.

Figure 15 shows that, as Co content in the composite increases, $\sigma$ (saturation magnetization) increases in hydrocarbon selective region $(\mathrm{Cu} / \mathrm{Co}<1.5)$, whereas (Figure 16) saturation magnetization decreases with increasing Co content in the composite in the alcohol-methanol selective region $(\mathrm{Cu} / \mathrm{Co}>1.5)$. The correlation coefficients $(\mathrm{R}$-values) between $\sigma$ and percent cobalt content, clearly indicate that Co dominance is high in $\mathrm{Cu} / \mathrm{Co}<1.5$ samples, and low in samples with $\mathrm{Cu} / \mathrm{Co}>1.5$. This supports the view that $\mathrm{Co}$ is the predominant player in hydrocarbon selective catalysts, while both $\mathrm{Co}$ and $\mathrm{Cu}$ play synergistic roles in alcohol selestive catalysts, and $\mathrm{Cu}$ assumes a predominant role in methanol selective catalysts. 
Figure 17 shows that, as $\mathrm{Cu}$ content in the composite increases $\sigma$ has decreased in hydrocarbon selective region $(\mathrm{Cu} / \mathrm{Co}<1.5)$, while $\sigma$ increases with increasing $\mathrm{Cu}$ content in alcohol-methanol selective regions $(\mathrm{Cu} / \mathrm{Co}>1.5)$ (Figure 18). These observations lead us to believe that $\mathrm{Cu}$ promotes Co reduction in alcohol-methanol selective region, while in hydrocarbon selective region $\mathrm{Cu}$ might produce $\mathrm{CoCu}_{2} \mathrm{O}_{4}$ type spinels which are not easily amiable for reduction.

Figures 19 and 20 show the effect of chromia on both hydrocarbon and alcoholmethanol selective catalysts. Saturation magnetization has consistently decreased with increasing $\mathrm{Cr}$ content in the composite. It appears that $\mathrm{Cr}$ in the form of Chromia essentially plays the role of a neutral support dispersing cobalt and copper uniformly.

Figure 21 shows that, as $\mathrm{Cu} / \mathrm{Co}$ ratio increases, $\sigma$ decreases in hydrocarbon selective region $(\mathrm{Cu} / \mathrm{Co}<1.5)$, and $\sigma$ increases with increasing $\mathrm{Cu} / \mathrm{Co}$ ratio in alcoholmethanol selective region $(\mathrm{Cu} / \mathrm{Co}>1.5)$ (Figure 22). This observation lends further support to the earlier inference on the role of copper in the composite.

A comparison between the saturation magnetization values of reduced samples and $\mathrm{CO}$ adsorbed samples presented in Table 9 indicate that $\mathrm{CO}$ adsorption in general decreases the magnetization in hydrocarbon selective catalysts while it leads to a slight increase in alcohol-methanol selective catalysts. This suggests that the direction of charge transfer between carbon monoxide and cobalt $\mathrm{Co} \Longleftrightarrow \mathrm{CO}$ is reversed in the two regions.

CO was physically desorbed from the adsorbed samples by placing the samples in a vacuum desiccator and evacuating continuously for several hours at room temperature. The magnetization results show no noticeable changes in the $\sigma$ values between the $C O$ adsorbed and the desorbed samples. This indicates that the adsorption process is not 
physi-sorption and more of chemi-sorption in nature and that most of the $\mathrm{CO}$ is chemically bound to the catalyst and might have formed cobalt carbonyls.

For the sample with the highest $\mathrm{Cu} / \mathrm{Co}$ ratio (sample $\mathrm{Cu} / \mathrm{Co} / \mathrm{Cr}-74 / 2 / 24, \mathrm{Cu} / \mathrm{Co}=$ 37), a dual phase in the magnetization curve is observed with a small hysteresis loop near the origin and a raising paramagnetic straight line. The increased $\mathrm{Cu}$ content could have lead to the formation of solid solution of $\mathrm{Co}$ into $\mathrm{Cu}$.

When we compare the $\sigma$ values of $\mathrm{CO}$ adsorbed and Syngas adsorbed catalysts, $\sigma$ value decreases when CO only is exposed. When Syngas is exposed $\sigma$ values did not change significantly and remained close to the reduced sample values. It appears that the presence of hydrogen prevents strong chemical interaction between $\mathrm{CO}$ and $\mathrm{Co}$ and inhibits the formation of cobalt carbonyls. 
Table 8: NMR Spectra of $\mathrm{Cu}-\mathrm{Co}-\mathrm{Cr}$ Catalysts with and without Adsorbed Carbon Monoxide and Syngas $\left(\mathrm{CO}+\mathrm{H}_{2}\right)$

\begin{tabular}{|c|c|c|c|c|c|c|c|c|c|c|c|c|c|c|c|}
\hline & & & & & & & & & & & & & & & \\
\hline & & & & & & & & \multicolumn{2}{|c|}{ NMR SPECTRA } & & & & & & \\
\hline SAMPLE & Culco & & & & & & & \multicolumn{3}{|c|}{ Cu.Co.Cr Catalysts } & & & & & \\
\hline I.D & RATIO & 210 & & 213 & & 215.5 & & 217 & 218.4 & 221 & & & & & \\
\hline $\mathrm{Cu} / \mathrm{Co} / \mathrm{Cr}$ & & & & FCC & & $\ldots \ldots$ & FAULT & LINES & $\ldots \ldots$ & HCP & & & & & \\
\hline & & & & & & & & & & & & & & & \\
\hline & & & & & & & & & & & & & & & \\
\hline $32 / 64 / 4$ & 0.5 & 210.8 & & 213.3 & & & 216.1 & & 218.4 & 220.9 & 222.5 & 223.3 & & & \\
\hline $32 / 64 / 4$ & $+\mathrm{CO} \quad 0.5$ & 210.8 & & 213.1 & & & 216 & & & 220.7 & 222.3 & & & & \\
\hline & & & & & & & & & & & & & & & \\
\hline 37137126 & 1.0 & 211.1 & & 213.6 & & & 216.6 & & & & 221.7 & 223.41 & & 225.3 & \\
\hline $37137 / 26$ & $+C 0 \quad 1.0$ & 211.1 & & 213.6 & & & & & & & & 223.7 & & 225 & \\
\hline & & & & & & & & & & & & & & & \\
\hline $43 / 28 / 29$ & 1.5 & 211.6 & & 213.8 & & & & & & & & 223.7 & & & \\
\hline $43 / 28 / 29$ & $+\mathrm{CO}$ & 211.2 & & 213.8 & & & & & & & & 223.7 & & & \\
\hline 5890113 & 20 & 2111 & & 213 a & & & & & & & & 2239 & & & \\
\hline $58 / 29 / 13$ & $+\mathrm{CO}$ & & & 213.7 & & & & & & & & $\frac{223.9}{223.8}$ & & & \\
\hline & & & & & & & & & & & & & & & \\
\hline $54 / 22 / 24$ & 2.5 & & & & & & & & & & & 223.7 & & & \\
\hline $54 / 22 / 24$ & $+\mathrm{CO} \quad 2.5$ & & & & & & & & & & & 223.7 & & & \\
\hline $60 / 20 / 20$ & 3.0 & 2118 & & 213.7 & & & & & & & & 223,8 & & & \\
\hline $\begin{array}{l}\frac{60 / 20 / 20}{60 / 20 / 20} \\
\end{array}$ & $\begin{array}{l}3.0 \\
+C 0 \quad 3.0 \\
\end{array}$ & & & $\frac{210.4}{213.4}$ & & & & & & & & 223.9 & & & \\
\hline & & & & & & & & & & & & & & & \\
\hline $74 / 21 / 5$ & 3.5 & 211 & 212.1 & 213.5 & 214.7 & & 216.6 & 217.8 & & & & 223.9 & & & \\
\hline $74 / 21 / 5$ & $+\mathrm{CO} \quad 3.5$ & & & & & & & & & & & & 224.5 & & \\
\hline & & & & & & & & & & & & & & & \\
\hline $63 / 16 / 21$ & 4.0 & & 212.8 & & & & & & & & & & 224.8 & & 226.5 \\
\hline $63 / 16 / 21$ & $+\mathrm{CO} \quad 4.0$ & & 212.4 & & & & & & & & & & 224.8 & & 226.3 \\
\hline 20189 & 44 & & 215? & & 2943 & & & & & & & 223 & 20 & & 2063 \\
\hline $80 / 18 / 2$ & 4.4 & & 212.24 & & 214.3 & & & & & & & 220 & 224.4 & & 240.0 \\
\hline $17778 / 5$ & $+\mathrm{CO}+\mathrm{H} 2,0.2$ & 210.8 & & 213.4 & & & 210 & & 218.7 & & 221.9 & 223 & & & \\
\hline . & 5 & & & & & & & & & & & & & & \\
\hline $37 / 137126$ & $+\mathrm{CO}+\mathrm{H}_{2} 21,0$ & & & & & & & & & & & 223.3 & & 225 & 226.5 \\
\hline 791204 & $\mathrm{CO}+\mathrm{H} 237$ & & & & & & & & & & 224.7 & 223.1 & 224.8 & & 226.2 \\
\hline
\end{tabular}


Table 9: Magnetization Results of $\mathrm{Cu}-\mathrm{Co}-\mathrm{Cr}$ Catalysts with and without adsorbed, desorbed $\mathrm{CO}$ and Syngas.

\begin{tabular}{|c|c|c|c|c|c|c|c|c|c|c|c|}
\hline Cu-Co-Cr & $\mathrm{Cu} / \mathrm{Co}$ & $\sigma$ & $\mathrm{Hc}$ & $\begin{array}{c}c \\
(c 0)\end{array}$ & $\begin{array}{l}\mathrm{Hc} \\
(\mathrm{CO})\end{array}$ & $\begin{array}{c}\sigma \\
\left(\mathrm{CO}+\mathrm{H}_{2}\right)\end{array}$ & $\begin{array}{c}\mathrm{Hc} \\
\left(\mathrm{CO}+\mathrm{H}_{2}\right)\end{array}$ & $\begin{array}{c}\sigma \\
(\mathrm{COd})\end{array}$ & $\begin{array}{c}\mathrm{Hc} \\
(\mathrm{COd})\end{array}$ & $\begin{array}{c}\sigma \\
\left(\mathrm{COH}_{2} \mathrm{~d}\right)\end{array}$ & $\begin{array}{c}\mathrm{Hc} \\
\left(\mathrm{COH}_{2} \mathrm{~d}\right)\end{array}$ \\
\hline $17-78-5$ & 0.2 & 117 & 672 & 84 & 463 & 128 & 429 & & 477 & 126 & 442 \\
\hline $37-37-26$ & 1 & 40 & 484 & 15 & 436 & 35 & 244 & 12.6 & 449 & 39 & 252 \\
\hline $74-2-24$ & 37 & D.P. & 203 & D.P. & 105 & D.P. & 117 & D. P. & 145 & D. P. & 66 \\
\hline & & & & & & & & & & & \\
\hline $8-71-21$ & 0.1 & 87 & 403 & 70 & 271 & & & 64 & 423 & & \\
\hline $32-64-04$ & 0.5 & 107 & 299 & 82 & 328 & & & & & & \\
\hline $43-28-29$ & 1.5 & 23 & 254 & 29 & 272 & & & & & & \\
\hline $43-26-31$ & 1.7 & 47 & 519 & 5.8 & 380 & & & 9.2 & 378 & & \\
\hline $58-29-13$ & 2 & 48 & 381 & 42 & 346 & & & 42 & 373 & & \\
\hline $54-22-24$ & 2.5 & 65 & 424 & 19 & 371 & & & 10 & 327| & & \\
\hline $60-20-20$ & 3 & 16 & 198 & 23 & 244 & & & 23 & 257| & & \\
\hline 74-21-5 & 3.5 & 73 & 411 & 70 & 347 & & & 68 & 368 & & \\
\hline $63-16-21$ & 4 & 12 & 306 & 17 & 312 & & & 17 & 322 & & \\
\hline 80-18-02 & 4.5 & 76 & 289 & 105 & 252 & & & 97 & 258 & & \\
\hline
\end{tabular}




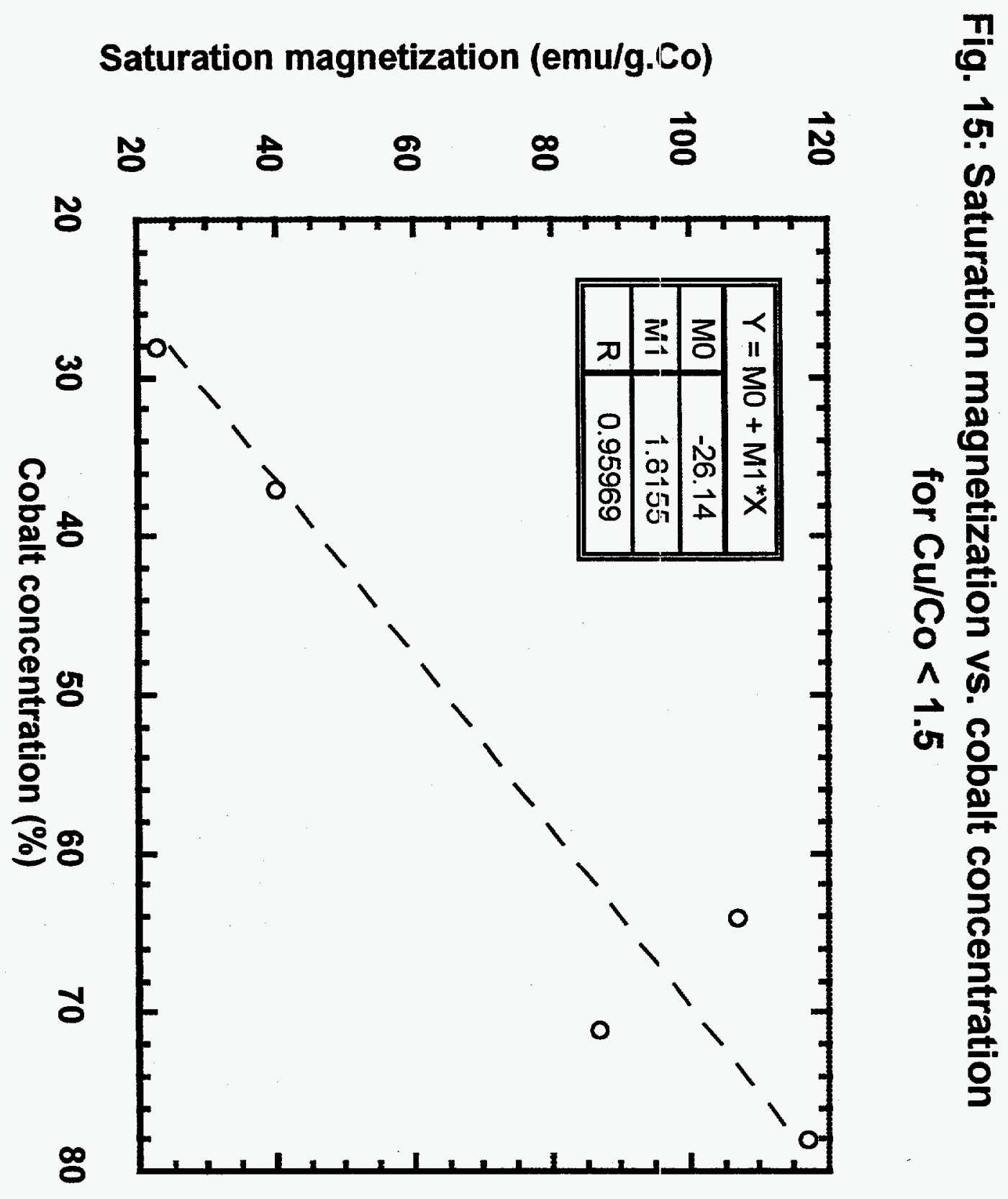




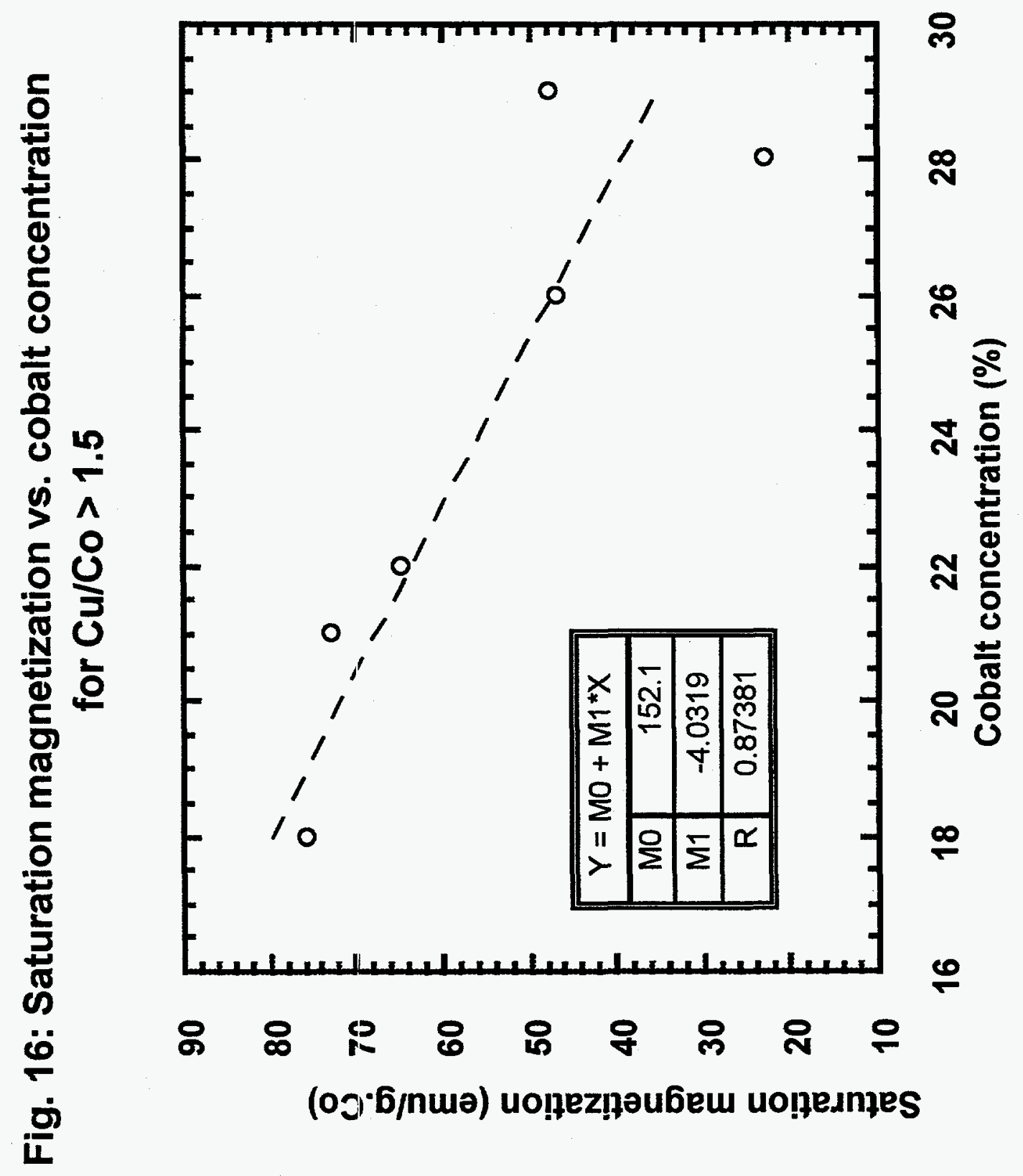




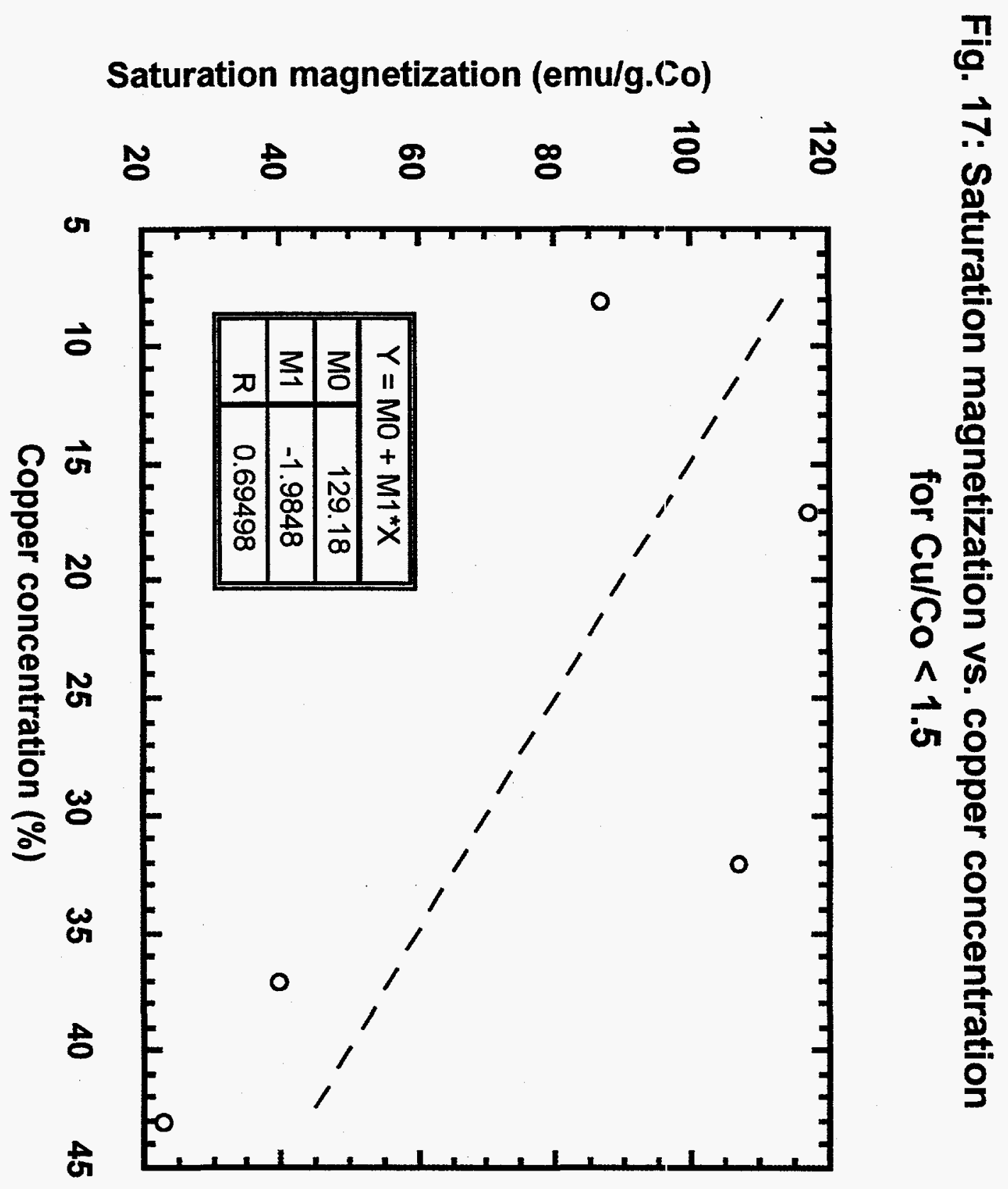




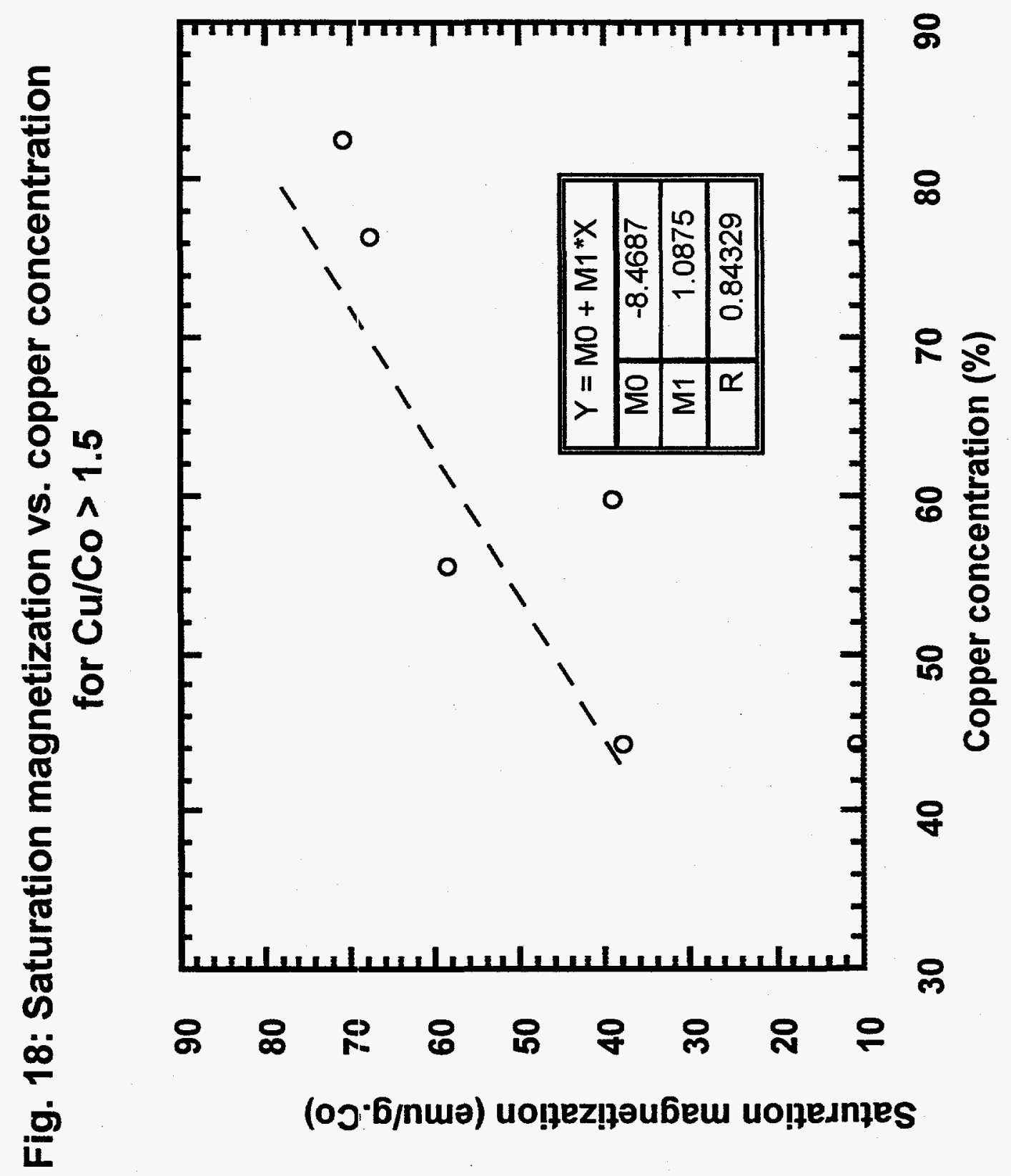


Fig. 19: Saturation magnrtization vs. chromium concentration for $\mathrm{Cu} / \mathrm{Co}<1.5$

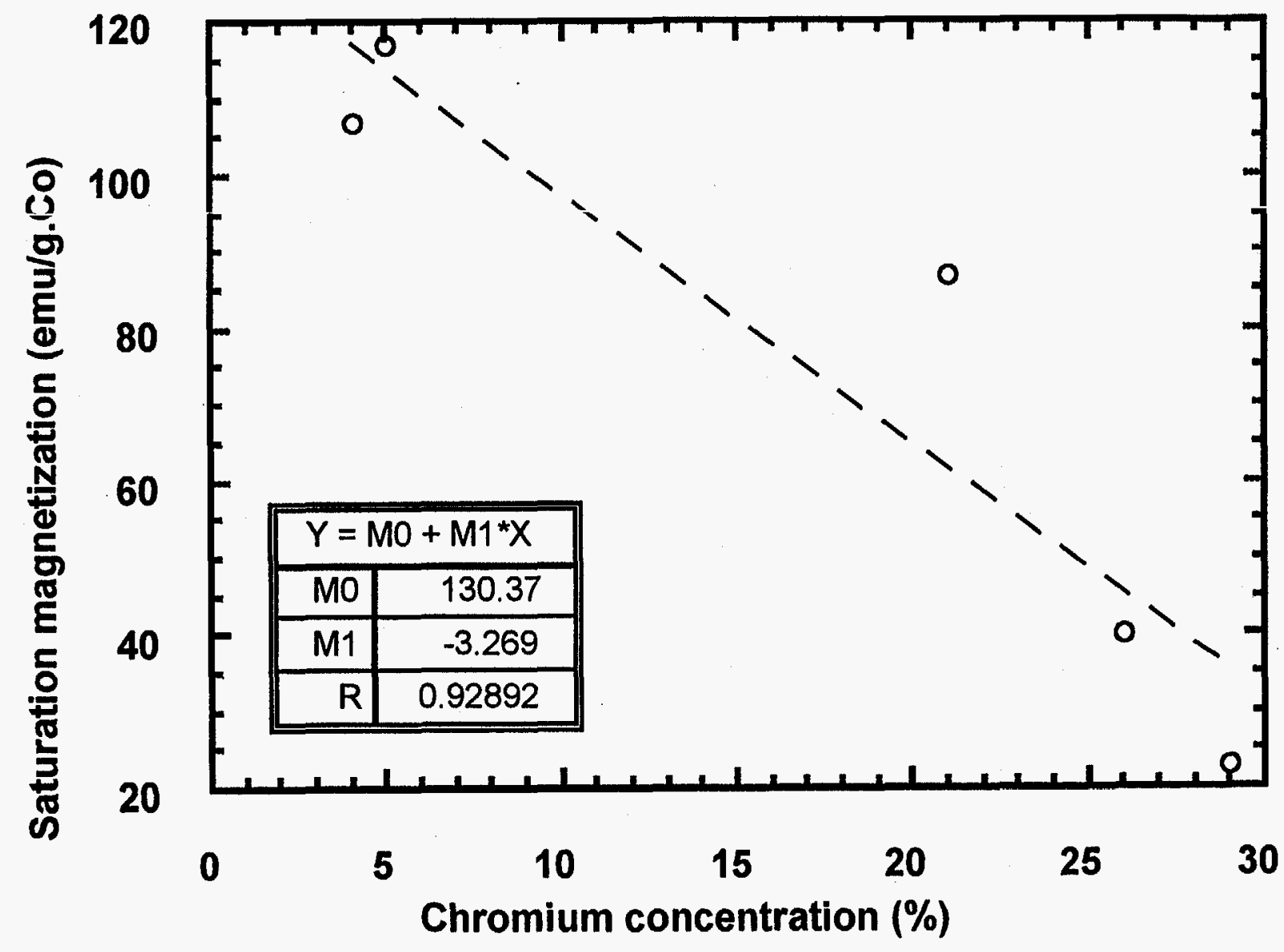


Fig. 20: Saturation magnetization vs. chromium concentration for $\mathrm{Cu} / \mathrm{Co}>1.5$

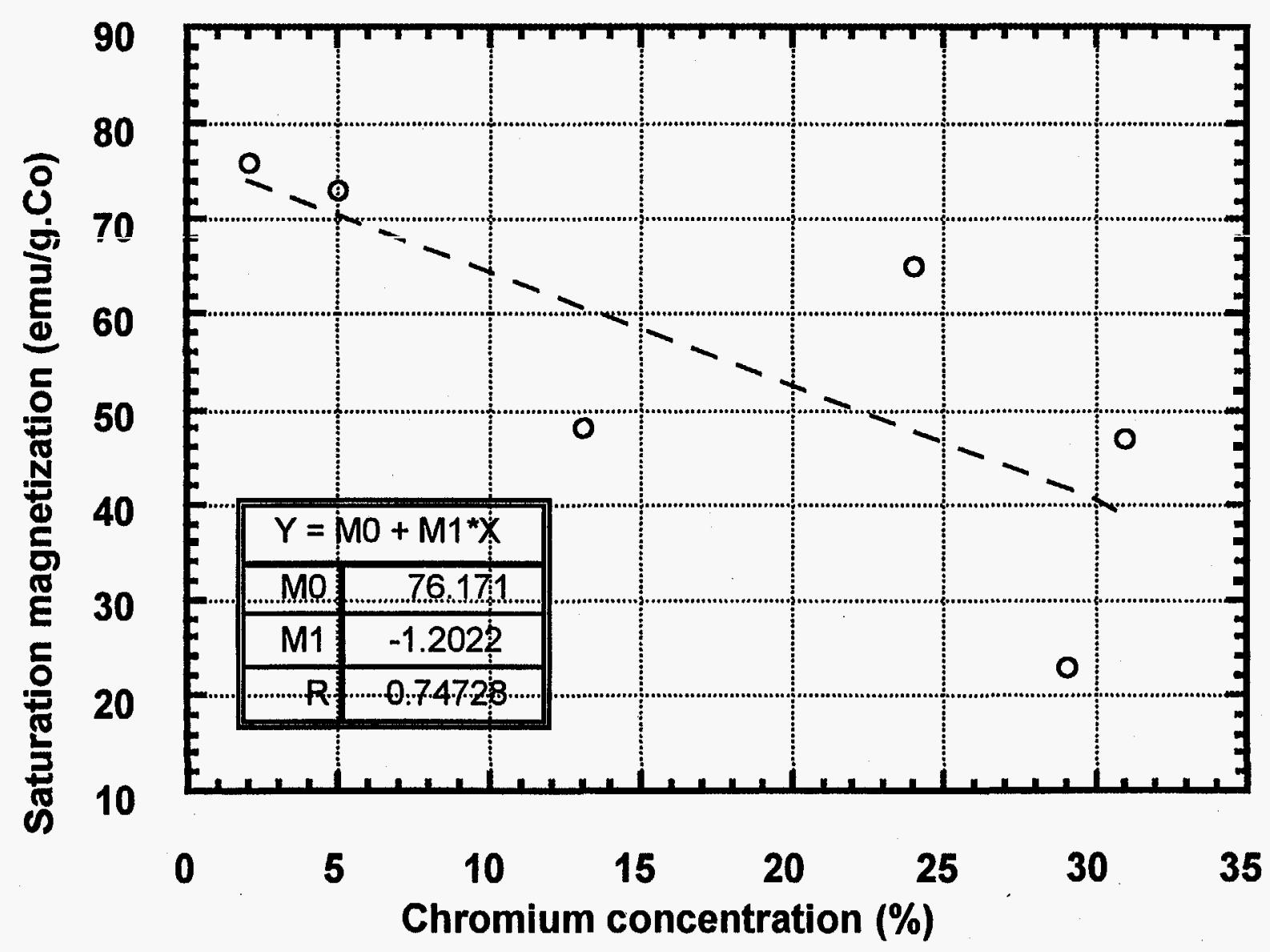


Fig. 21: Saturation magnetization vs. Cu/Co ratio for $\mathrm{Cu} / \mathrm{Co}<1.5$

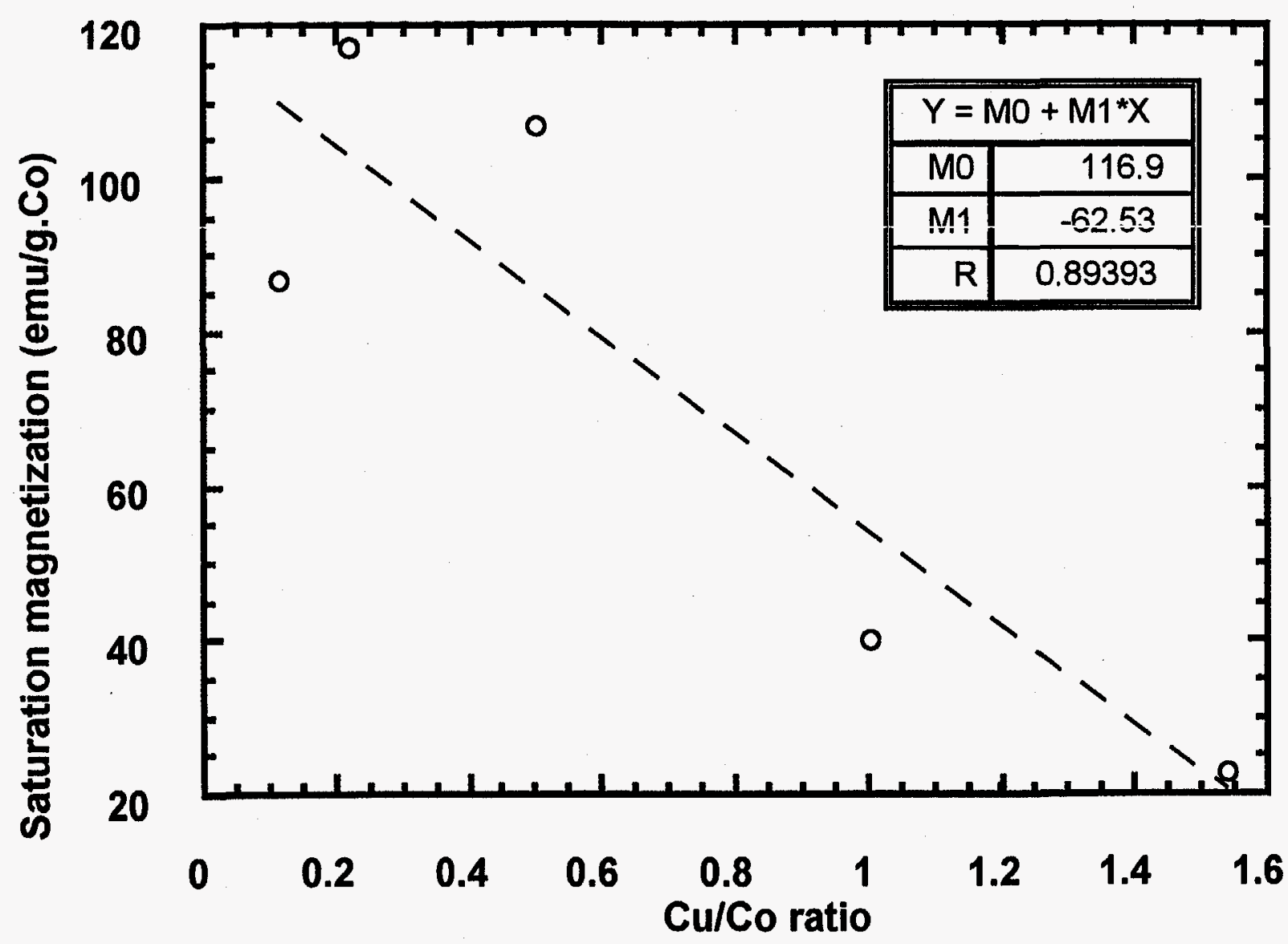




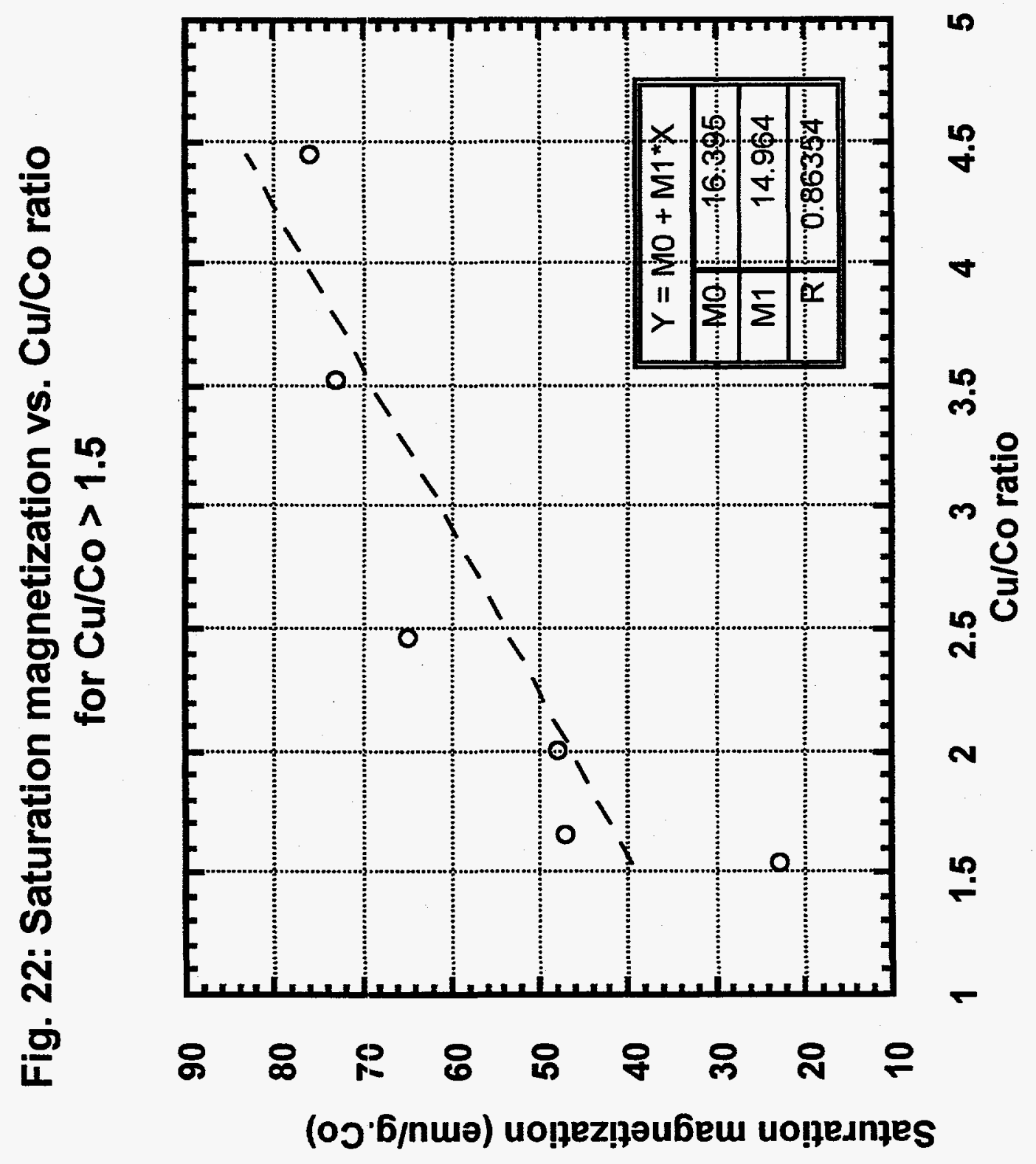




\section{FTIR Studies}

A comprehensive FTIR investigation on the catalysts was undertaken to unfold the picture of the gas-metal interactions. Our magnetization studies have provided valuable information on the changes in the magnetic character of the ferromagnetic metal due to CO adsorption. The FTIR results showing the effect of the adsorbate-adsorbent interactions on the stretching frequencies of the $\mathrm{CO}$ vibrational modes are presented in this section.

A series of 10 calcined samples with $0.5<\mathrm{Cu} / \mathrm{Co}<5$ were loaded in the sample cup of the environmental chamber of the FTIR spectrometer and were reduced in-situ before exposing to carbon monoxide. Carbon monoxide was admitted at room temperature, and while flowing $\mathrm{CO}$, temperature was increased gradually in steps of $50^{\circ} \mathrm{C}$ and FTIR spectra were recorded at each temperature up to $250^{\circ} \mathrm{C}$. We did not find any significant differences in the spectra taken at different temperatures. This suggests that gas-metal interactions might occur even at room temperature. The FTIR results are presented in Tables 10-11 and graphically depicted in Figures 23-26. Except for the single band in the region $820-900 \mathrm{~cm}^{-1}$, all the observed bands seem to arise from four distinct metal carbonate structures as suggested by A. A. Davydov [41].

\section{Suggested Structures:}

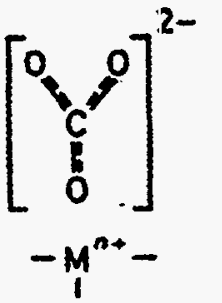

(III)

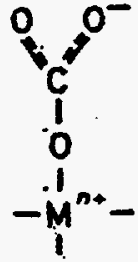

(III)<smiles>C[C@]12O[C@H](O)[C@@H](O1)O[N+]2(C)C</smiles>

(IVa)<smiles>[O][C@H](O)O[W]</smiles>

(IVb) 
The disproportination of carbon monoxide molecule produces $\mathrm{CO}_{2}$ ads according to Boudouard reaction

$$
\begin{gathered}
\mathrm{CO}_{\mathrm{ads}} \rightarrow \mathrm{C}^{*}+\mathrm{O}^{*} \\
2 \mathrm{CO}_{\mathrm{ads}} \rightarrow \mathrm{C}_{\mathrm{ads}}+\mathrm{CO}_{2}
\end{gathered}
$$

The $\mathrm{CO}_{2}$ produced is believed to stabilize in the matrix in the form of a) noncoordinated carbonates (structure II), b) Mono-dentate carbonates (structure III), and c) Bi-dentate carbonates (structures IVa and $b$ ) because of the reaction

$$
\mathrm{CO}_{2}+\mathrm{O}^{2-} \rightarrow \mathrm{CO}_{3}^{2-}
$$

The non-coordinated carbonates generate bands in the regions: $1420-1450 \mathrm{~cm}^{-1}\left(\mathrm{vCO}_{3}{ }^{2-}\right)$ and $1020-1090 \mathrm{~cm}^{-1}\left(\mathrm{v}_{\mathrm{s}} \mathrm{CO}_{3}{ }^{2-}\right)$, monodentate carbonate structures produce bands in the region $1470-1530 \mathrm{~cm}^{-1}\left(v_{\mathrm{as}} \mathrm{COO}^{-}\right), 1370-1300 \mathrm{~cm}^{-1}\left(v_{\mathrm{s}} \mathrm{COO}^{-}\right)$, and $1040-1080 \mathrm{~cm}^{-1}(\mathrm{VC}-\mathrm{O})$, and bidentate carbonate structures produce bands in the region $1530-1620 \mathrm{~cm}^{-1}(\mathrm{vC}=0)$, $1250-1270 \mathrm{~cm}^{-1}\left(v_{\mathrm{as}} \mathrm{COO}\right)$, and 1020-1030 $\mathrm{cm}^{-1}\left(v_{\mathrm{s}} \mathrm{COO}\right)$ for IVA type and $1620-1670$ $\mathrm{cm}^{-1}(v \mathrm{C}=0), 1220-1270 \mathrm{~cm}^{-1}\left(v_{\mathrm{as}} \mathrm{COO}\right), 980-1020 \mathrm{~cm}^{-1}\left(v_{\mathrm{s}} \mathrm{COO}\right)$ for IVB type. Table 10 shows the observed stretching frequencies of $\mathrm{CO}$ bands as $\mathrm{Cu} / \mathrm{Co}$ metal ratio increases from 0.5 to 5.0 . Since the $860 \pm 40 \mathrm{~cm}^{-1}$ band consistently appears for all metal ratios of $\mathrm{Cu} / \mathrm{Co} / \mathrm{Cr}$, and $\mathrm{Co} / \mathrm{Cr}$ composite catalysts and absent in all $\mathrm{Cu} / \mathrm{Co}$ composite catalysts suggests that this may be due to $\mathrm{CO}$ adsorption on Chromia. The bands due to structures II, III and IVb seem to be independent of the changes in the $\mathrm{Cu} / \mathrm{Co}$ intermetallic ratio. The ground state electronic configuration of $\mathrm{CO}$ is [42]

$$
1 \sigma^{2} 2 \sigma^{2} 3 \sigma^{2} 4 \sigma^{2} 1 \pi^{4} 5 \sigma^{2}
$$


Broden et.al., [43] consider that the $5 \sigma$ orbital of $\mathrm{CO}$ is essentially non-bonding with respect to the carbon and oxygen and therefore donation from the $5 \sigma$ orbital to the metal should not affect greatly the C-O bond strength /stretching frequency. As such in these structures, the gas-metal interaction could be through the overlap of $5 \sigma \mathrm{CO}$ orbital and $d$ orbitals of the metal. Our magnetization data support this view that charge transfer occur from $\mathrm{CO}$ to $\mathrm{Co}$, as evidenced by the drop in the magnetic moment of $\mathrm{Co}$.

The behavior of structure IVa is unique and closely parallels the magnetization results which show an increase in magnetic moment for the alcohol selective catalysts. The frequency increases from $1544 \mathrm{~cm}^{-1}$ for $\mathrm{Cu} / \mathrm{Co} \leq 1$ to $1604 \mathrm{~cm}^{-1}$ for $\mathrm{Cu} / \mathrm{Co}=4$ and drops for $\mathrm{Cu} / \mathrm{Co}$ ratios $>4$. An increase in magnetic moment is possible when the metal looses electron to the adsorbate. $\mathrm{CO}$ is considered as a typical $\pi$ acceptor. The back donation of electrons from the metal surface orbitals to the antisymmetric unoccupied $2 \pi^{*}$ of $\mathrm{CO}$ would result in lowering of the $\mathrm{CO}$ stretching frequency [44]. However our results show an increase in the $\mathrm{CO}$ stretching frequency in the alcohol region. Chini and Co-workers [45] report that when the number of metal atoms in a cluster increase, the stretching frequencies of $\mathrm{CO}$ also increase. The magnetization results clearly indicate that cobalt is well reduced in the alcohol selective catalysts increasing the number of metal atoms in given cluster. Hence in structure IV a the cluster mechanism seems to be responsible for observed increase in the frequencies instead of a drop as suggested by Dalman et.al. [46]. 


\section{Vibrational frequencies of $\mathrm{Cu}-\mathrm{Co}-\mathrm{Cr}$ Composites}

Figure 23

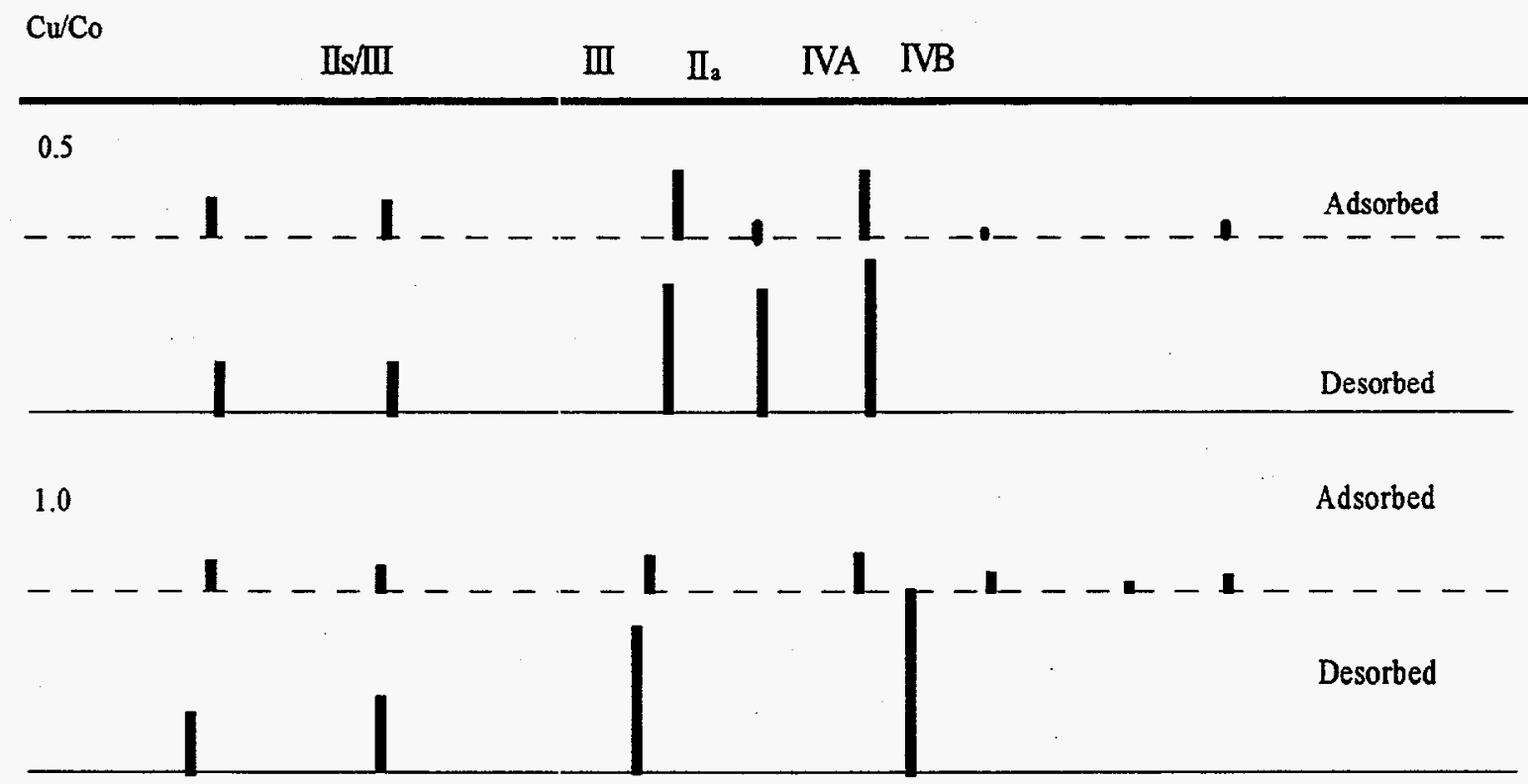

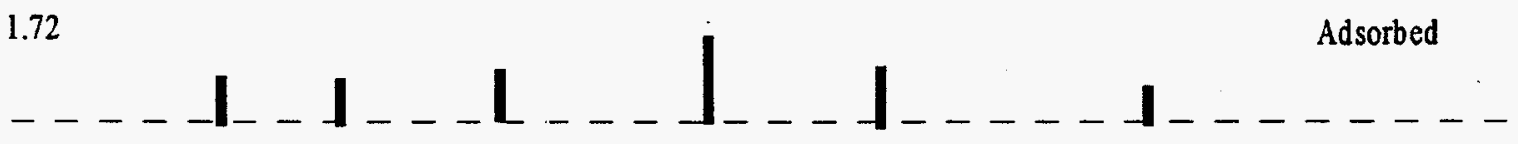

Desorbed

2.0

Adsorbed

$\ldots \ldots \ldots \ldots+\ldots \ldots \ldots$

Desorbed

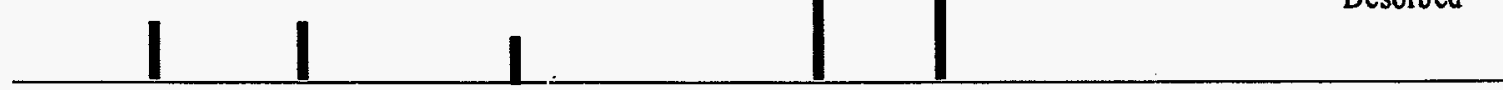

2.5

Adsorbed

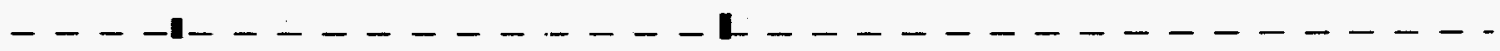

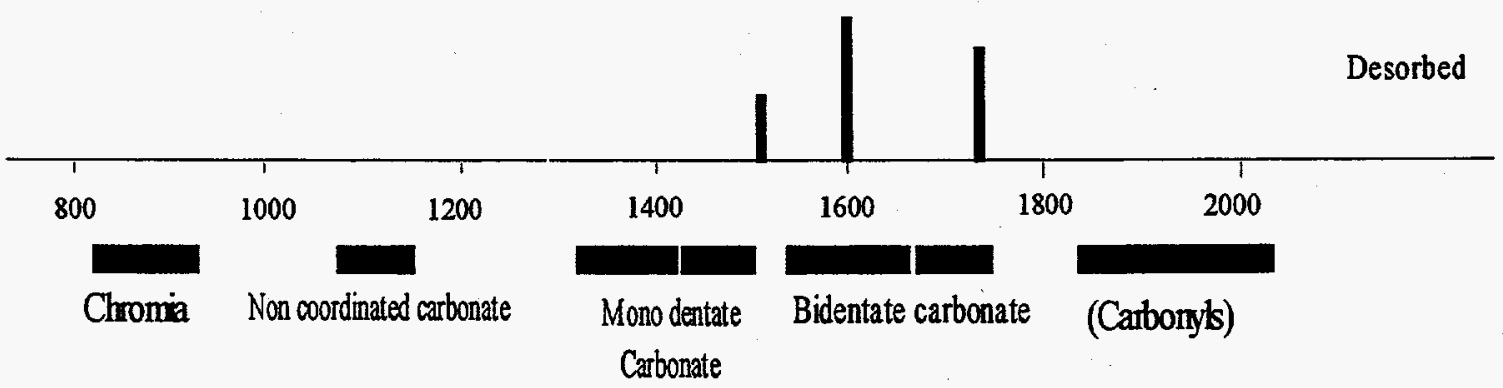




\section{Figure 24 Vibrational frequencies of $\mathrm{Cu}-\mathrm{Co}$-Cr Composites}
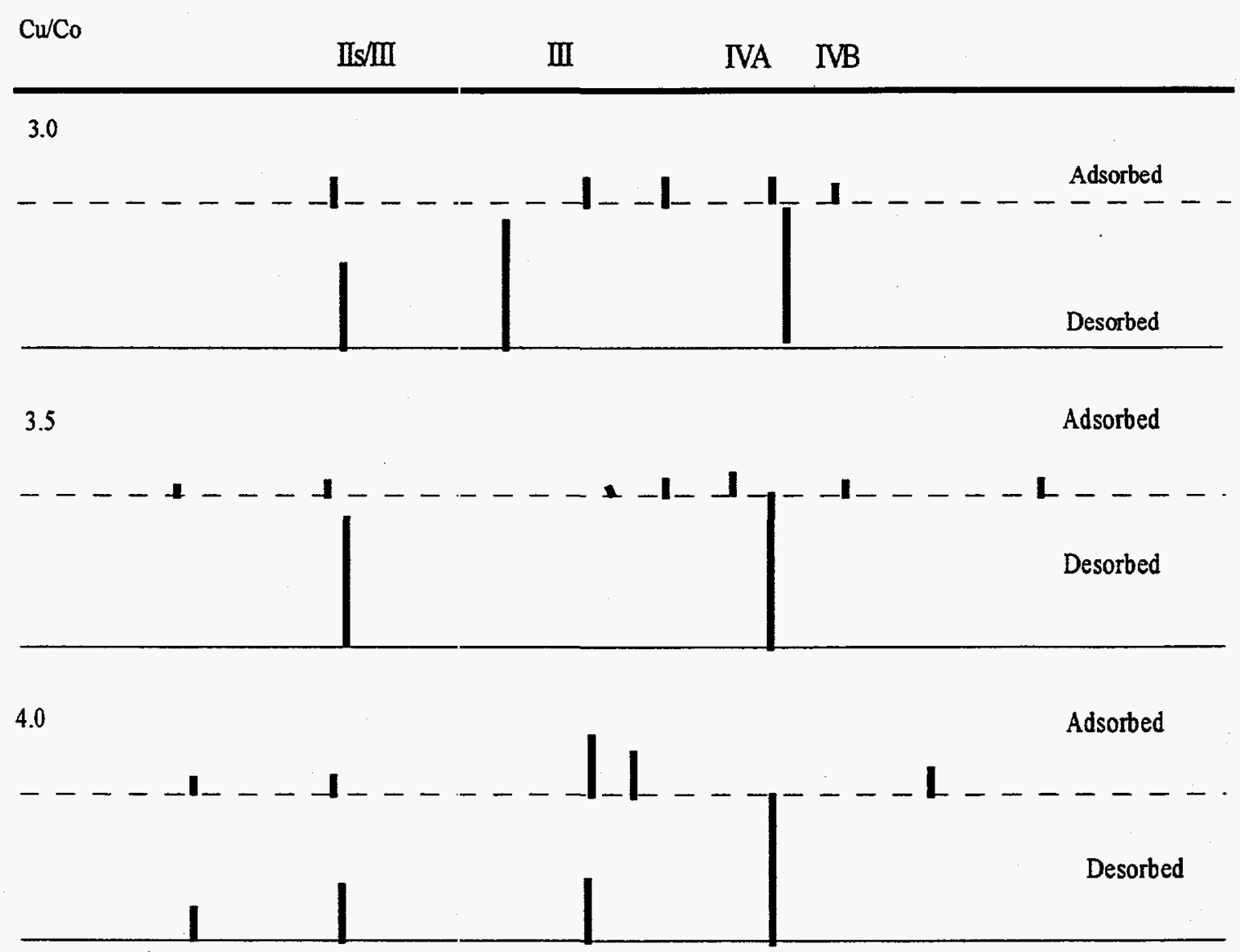

Adsorbed

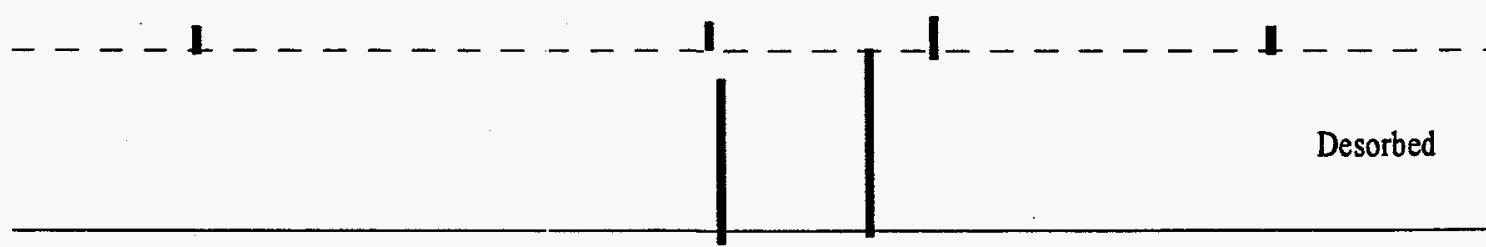

5.0

Adsorbed

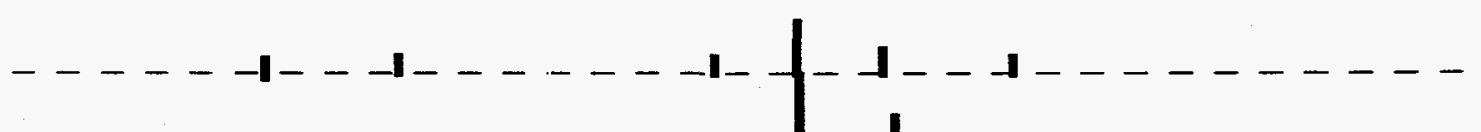

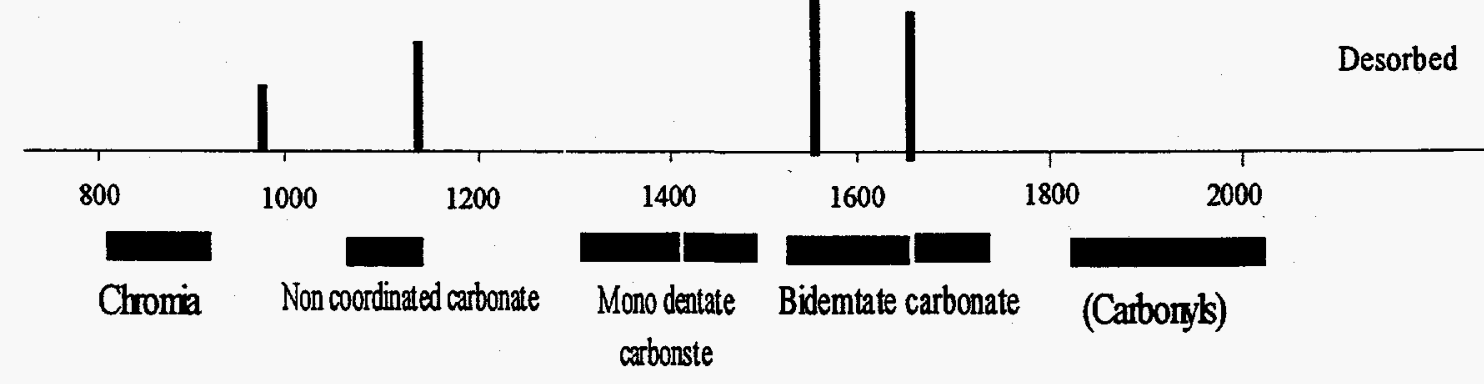




\section{Comparison of Adsorbed Frequencies}

Figure 25

\section{(Effect of Catalyst Composition)}

IIs/III III Ila IVA IVB

$\mathrm{Co} / \mathrm{Cr}(0.5)$

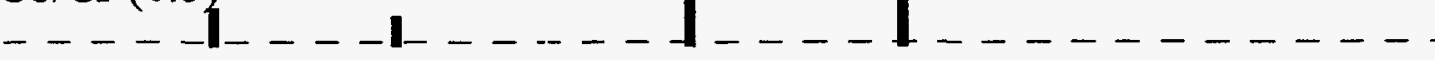

$\mathrm{Co} / \mathrm{Cu}(0.5)$

- - - - - - - - - - - L - $-\ldots$

$\mathrm{Co} / \mathrm{Cr} / \mathrm{Cu}(0.5)$

1

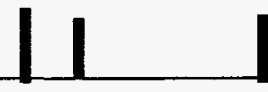

$\mathrm{Co} / \mathrm{Cr}(3.0)$

$\ldots-\ldots \ldots \ldots \ldots \ldots \ldots$

$\mathrm{Co} / \mathrm{Cu}(3.0)$

$\mathrm{Co} / \mathrm{Cr} / \mathrm{Cu}(3.0)$

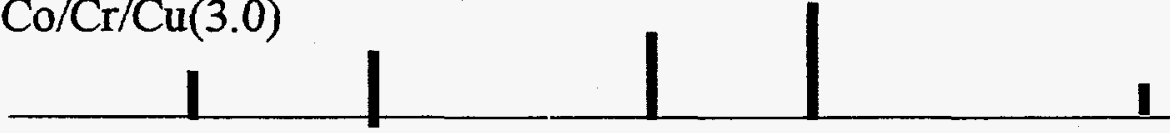

$\mathrm{Co} / \mathrm{Cr}(8.0)$

$-\ldots-\ldots+\ldots \ldots \ldots \ldots \ldots$

$\mathrm{Co} / \mathrm{Cu}(8.0)$

$\ldots \ldots \ldots-\ldots-\ldots-\ldots-\ldots-1 \ldots-\ldots$

$\mathrm{Co} / \mathrm{Cr} / \mathrm{Cu}(8.0)$

\begin{tabular}{l|l}
$\mathrm{Co} / \mathrm{Cr}(16.0)$ \\
$\ldots-\ldots+\left.\left.\ldots\right|_{-}\right|_{\ldots}$
\end{tabular}

$\mathrm{Co} / \mathrm{Cu}(16.0)$

$\mathrm{Co} / \mathrm{Cr} / \mathrm{Cu}(16.0)$

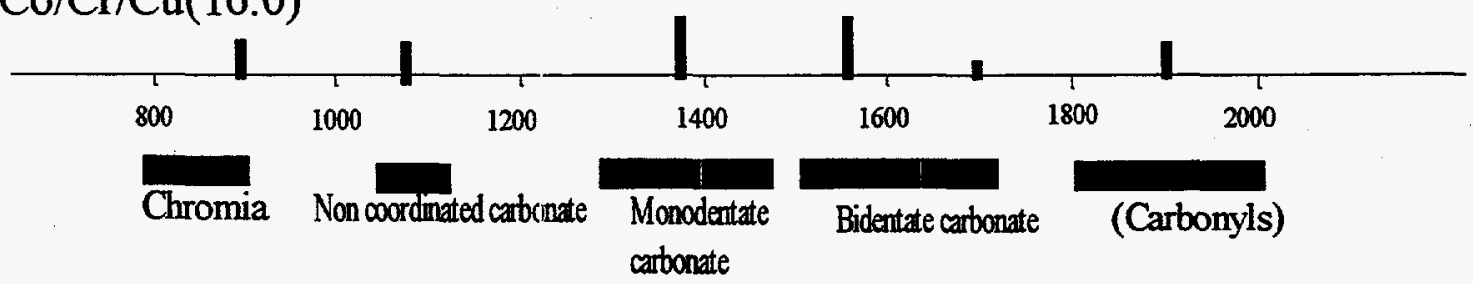




\section{Comparison of Desorbed Frequencies}

Figure 26

(Effect of Catalyst Composition)

IIs/III III IIa IVA IVB

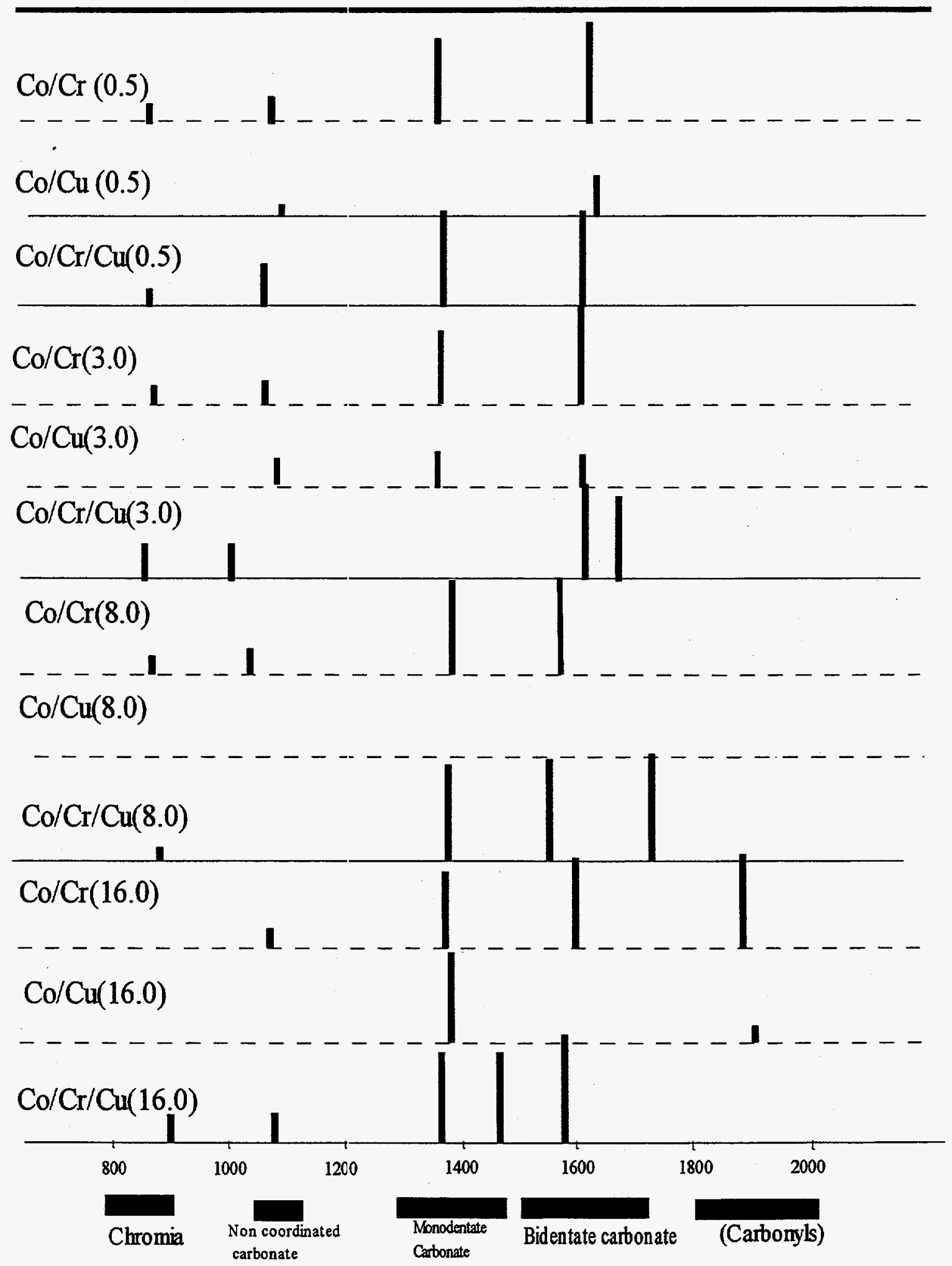


The high frequency bands in the $1900 \mathrm{~cm}^{-1}$ region appear only in the hydrocarbon and methanol selective catalysts. These are the only bands that disappeared upon desorption of gaseous CO. These may be due to the cobalt carbonyl formations, $\mathrm{Co}(\mathrm{CO})_{4}{ }^{-}$, that have transiently formed due to the $\mathrm{CO}_{2}$ stabilization on the matrix as $\mathrm{CO}_{3}{ }^{2-}$ species according to the reaction [47].

$$
2 \mathrm{Co}^{\circ}-\mathrm{CO}+2 \mathrm{O}^{2-} \rightarrow \mathrm{CO}_{3}{ }^{2-}+2 \mathrm{Co}(\mathrm{C} 0)_{4}^{-}
$$

This gradual disappearance is a consequence of gradual modification in the adsorbate-adsorbent interaction when the surface becomes covered by the adsorbed carbon (carbided surface). We designate these bands as physisorbed distinct from the carbonate like species bands which persist even after disorption and may be considered as resulting from the chemisorption process.

Three catalysts in the hydrocarbon, alcohol and methanol selective regions with $\mathrm{Cu} / \mathrm{Co}$ ratios $0.2,1$ and 37 were chosen to study the effect of syngas $\left(\mathrm{CO}+\mathrm{H}_{2}\right)$ interaction. Calcined samples were loaded into the sample cup of the DRIFT accessory and were reduced under hydrogen flow $(20 \mathrm{cc} / \mathrm{min})$ at $350^{\circ} \mathrm{C}$ for 18 hours. Hydrogen was outgassed while reducing the temperature to $25^{\circ} \mathrm{C}$. A mixture of $\mathrm{CO}+\mathrm{H}_{2}$ (carbon monoxide and hydrogen (50/50) supplied by Gulf Coast Airgas) was admitted at this temperature. While continuing syngas flow, the temperature was increased in steps of $50^{\circ} \mathrm{C}$ up until $250^{\circ} \mathrm{C}$ and FTIR spectra were taken at each temperature. $\mathrm{CO}+\mathrm{H}_{2}$ was desorbed by evacuating the chamber at $250^{\circ} \mathrm{C}$. While evacuating continuously, spectra were recorded in the reverse order. We did not observe any significant changes in the FTIR spectra due to temperature either in the adsorbed or desorbed species. 
Figures 27-28 represent the FTIR spectra of the adsorbed and desorbed species of the three samples. Table 12 lists the vibrational frequencies along with their assigments. For comparison, $\mathrm{CO}$ adsorbed frequencies were also shown. In all the three samples fewer vibrational modes were excited in syngas exposed spectra compared to the carbon monoxide only adsorbed spectra. In the hydrocarbon selective catalyst $(\mathrm{Cu} / \mathrm{Co}=0.2)$, when catalyst exposed to carbon monoxide only, carbonyls, mono dentate, and bidentate carbonates are formed. When the catalyst is exposed to $\mathrm{CO}+\mathrm{H}_{2}$ only, non-coordinated carbonates are formed and all other structures are suppressed,. In the mid range $(\mathrm{Cu} / \mathrm{Co}=$ 1), both monodentate and bidentate formation is not suppressed due to syngas unlike in hydrocarbons. Carbonyls formation seems to be suppressed in mid-range (alcohol selective catalyst), both in the presence of syngas or carbon monoxide. In the methanol and hydrocarbon selective regions only the presence of hydrogen seem to suppress the formation of carbonyls. Also in the methanol and hydrocarbon selective catalysts, the monodentate and bidentate carbonate formation seem to be transcient, whereas in alcohol catalysts, the bidentate carbonate: formation persist even after desorption. 
Table 10

Vibrational frequencies of $\mathrm{Cu}-\mathrm{Co}-\mathrm{Cr}$ Catalysts

\begin{tabular}{|c|c|c|c|c|c|c|c|c|}
\hline SAMPLE I & $\begin{array}{c}\text { Metal ratio } \\
\text { Cu/Co }\end{array}$ & $\begin{array}{l}\mathrm{CO} \text { on } \\
\mathrm{Cr}_{2} \mathrm{O}_{3} \\
\\
800-900 \\
\end{array}$ & $\begin{array}{c}v_{\mathrm{s}} \mathrm{CO}_{3}-/ v \mathrm{C}-\mathrm{O} \\
\text { II/III } \\
1040-1080 \\
\end{array}$ & $\begin{array}{c}v_{s} \mathrm{COO}^{-} \\
\text {III } \\
1300-1370 \\
\end{array}$ & $\begin{array}{c}v_{\mathrm{as}} \mathrm{CO}_{3}^{2-} \\
\| \\
1420-1450\end{array}$ & $\begin{array}{c}\mathrm{CO}=0 \\
\mathrm{IV} \mathrm{a} \\
1530-1620 \\
\end{array}$ & $\begin{array}{c}v C=0 \\
I V b \\
1620-1670\end{array}$ & $\begin{array}{c}\text { Carbonyls } \\
(\mathrm{CO})_{4}^{-} \\
1800-2000\end{array}$ \\
\hline $32-64-4$ & 0.5 & $\begin{array}{c}860 \\
(879)\end{array}$ & $\begin{array}{c}1056 \\
(1058) \\
\end{array}$ & $\begin{array}{c}1358 \\
(1344) \\
\end{array}$ & $\begin{array}{c}1434 \\
(1446) \\
\end{array}$ & $\begin{array}{c}1544 \\
(1556) \\
\end{array}$ & 1673 & 1928 \\
\hline $37-37-26$ & 1.0 & $\begin{array}{c}859 \\
(845) \\
\end{array}$ & $\begin{array}{c}1056 \\
(1058) \\
\end{array}$ & $\begin{array}{c}1328 \\
(1316) \\
\end{array}$ & - & $\begin{array}{c}1544 \\
(1598) \\
\end{array}$ & 1673 & 1925 \\
\hline $43-28-29$ & 1.5 & $\begin{array}{c}898 \\
(877)\end{array}$ & $\begin{array}{c}1024 \\
(1031)\end{array}$ & $\begin{array}{c}1392 \\
(1377)\end{array}$ & - & $\begin{array}{c}1580 \\
(1603)\end{array}$ & - & - \\
\hline $58-29-13$ & 2.0 & $\begin{array}{c}822 \\
(834)\end{array}$ & 1020 & $\begin{array}{c}1392 \\
(1359)\end{array}$ & - & $\begin{array}{c}1598 \\
(1648)\end{array}$ & - & - \\
\hline $54-22-24$ & 2.5 & 850 & - & $(100)$ & $\begin{array}{c}1415 \\
(1453)\end{array}$ & $(167 \overline{6,1538)}$ & - & - \\
\hline $60-20-20$ & 3.0 & - & $\begin{array}{c}1036 \\
(1035)\end{array}$ & $\begin{array}{c}1352 \\
(1344)\end{array}$ & 1454 & $\begin{array}{c}1598 \\
(1606)\end{array}$ & 1670 & - \\
\hline $74-21-5$ & 3.5 & $\begin{array}{c}823 \\
(840)\end{array}$ & $\begin{array}{c}1017 \\
(1044)\end{array}$ & 1383 & 1455 & $\begin{array}{c}1539 \\
(1591)\end{array}$ & 1688 & 1937 \\
\hline $63-16-21$ & 4.0 & $\begin{array}{l}850 \\
(844)\end{array}$ & $\begin{array}{c}1019 \\
(1035)\end{array}$ & $\begin{array}{c}1351 \\
(1352)\end{array}$ & 1408 & $\begin{array}{c}1604 \\
(1588)\end{array}$ & - & - \\
\hline $80-18-2$ & 4.5 & 823 & - & $\begin{array}{c}1352 \\
(1371)\end{array}$ & - & $\begin{array}{c}1597 \\
(1518)\end{array}$ & - & 1941 \\
\hline $50-10-40$ & 5.0 & $\begin{array}{c}878 \\
(878)\end{array}$ & $\begin{array}{c}1025 \\
(1025)\end{array}$ & - & $\begin{array}{c}1445 \\
(1445)\end{array}$ & $\begin{array}{c}1541 \\
(1545)\end{array}$ & 1672 & - \\
\hline
\end{tabular}




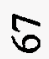

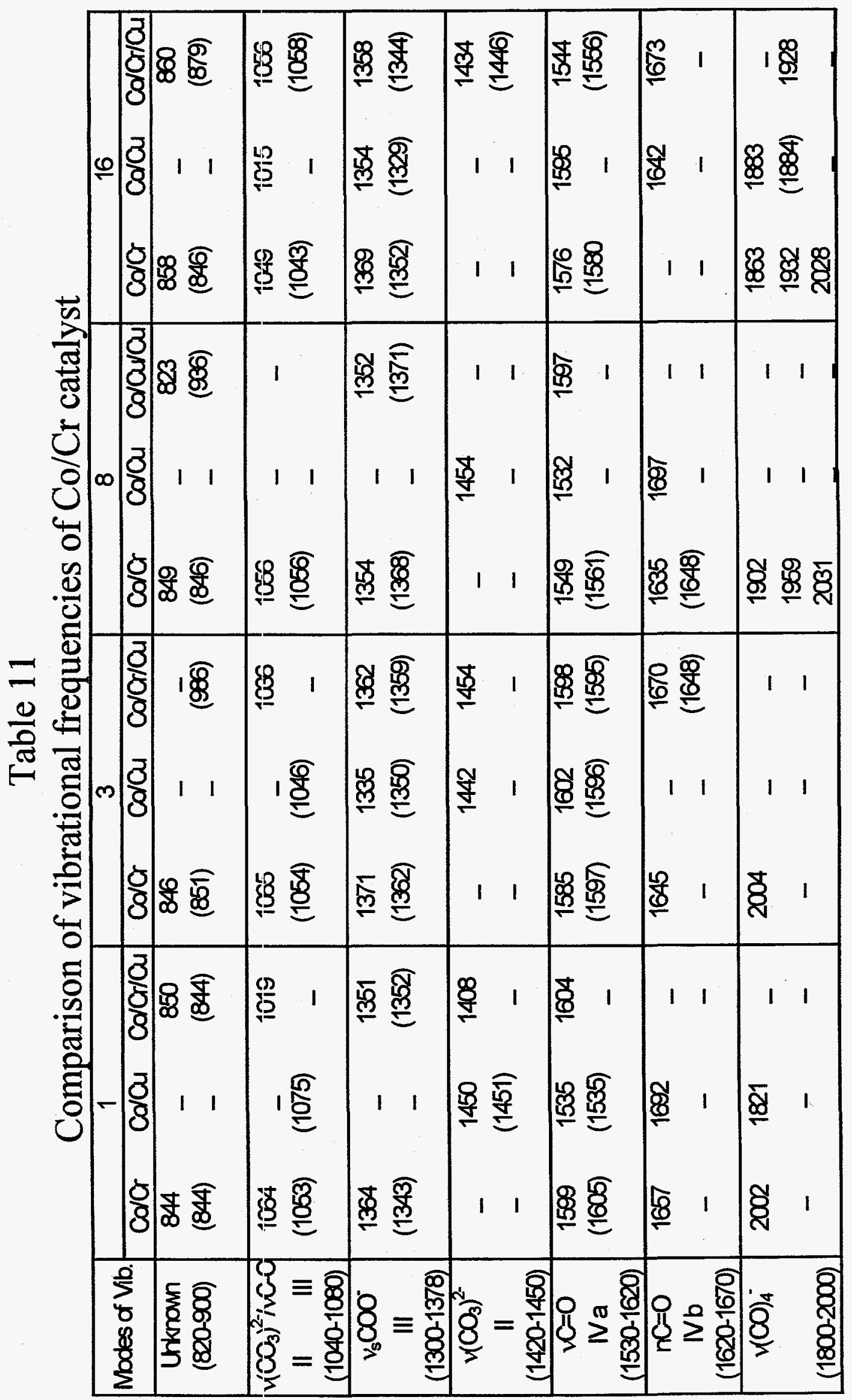


Figure 27: Comparison of Vibrational Frequencies of $\mathrm{CO}$ and $\mathrm{CO}+\mathrm{H2}$ Adsorbed Species $\mathrm{Cu} / \mathrm{Co} / \mathrm{Cr}$ $\begin{array}{lllll}\text { IIs/III III } & \Pi_{\mathbf{a}} & \text { IVA } & \text { IVB }\end{array}$
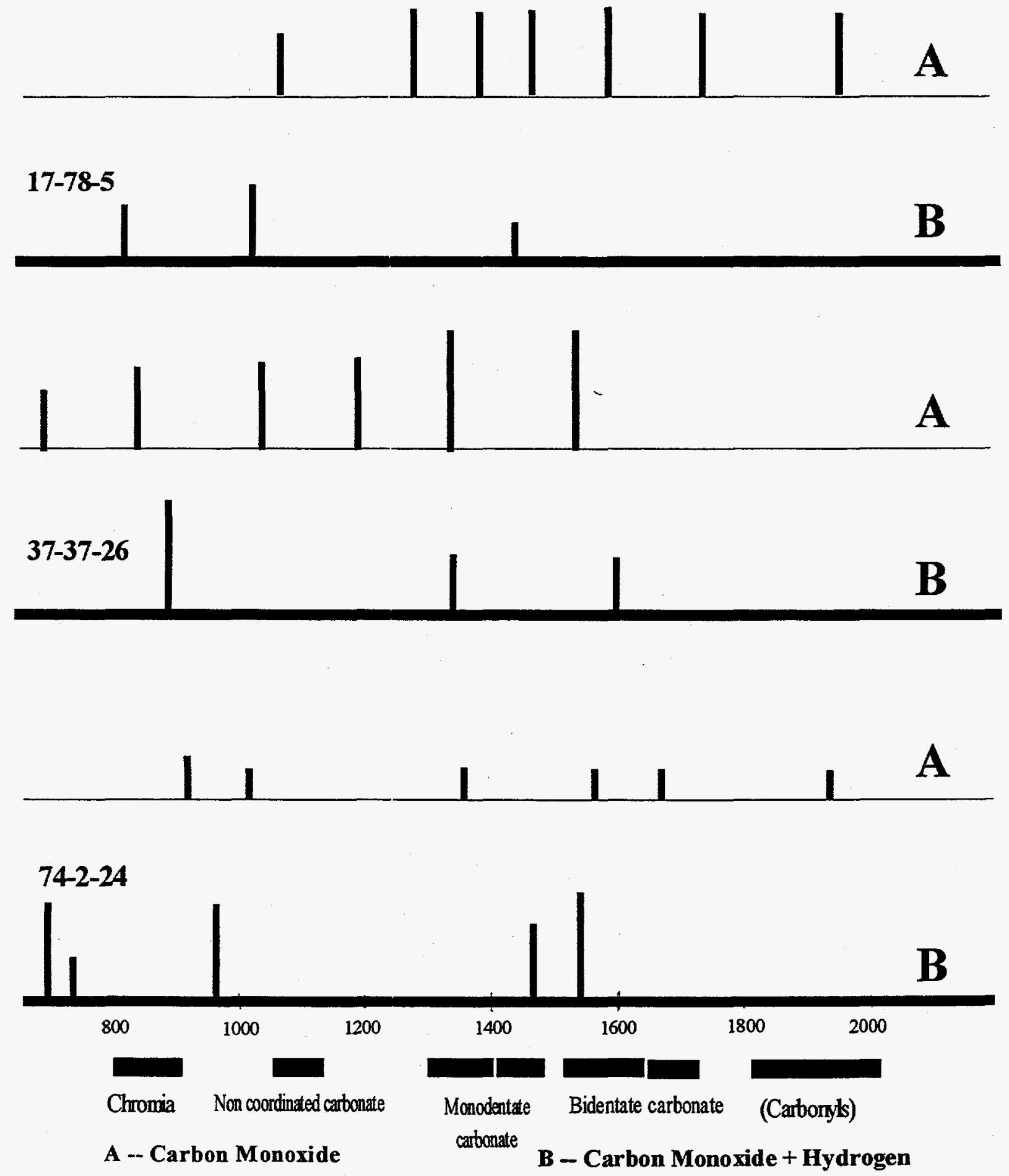
Figure 28: Comparison of Vibrational Frequencies of $\mathrm{CO}$ and $\mathrm{CO}+\mathrm{H} 2$ Desorbed Species

\begin{tabular}{|c|c|}
\hline $\mathrm{C} u / \mathrm{Co}^{2} / \mathrm{Cr}$ & $\begin{array}{l}v_{s} \mathrm{CO}_{3}^{-1} / \mathrm{vCO} \\
\text { IIs/III }\end{array}$ \\
\hline
\end{tabular}

\begin{tabular}{ll|l|} 
& $\mathbf{A}$ \\
\hline
\end{tabular}
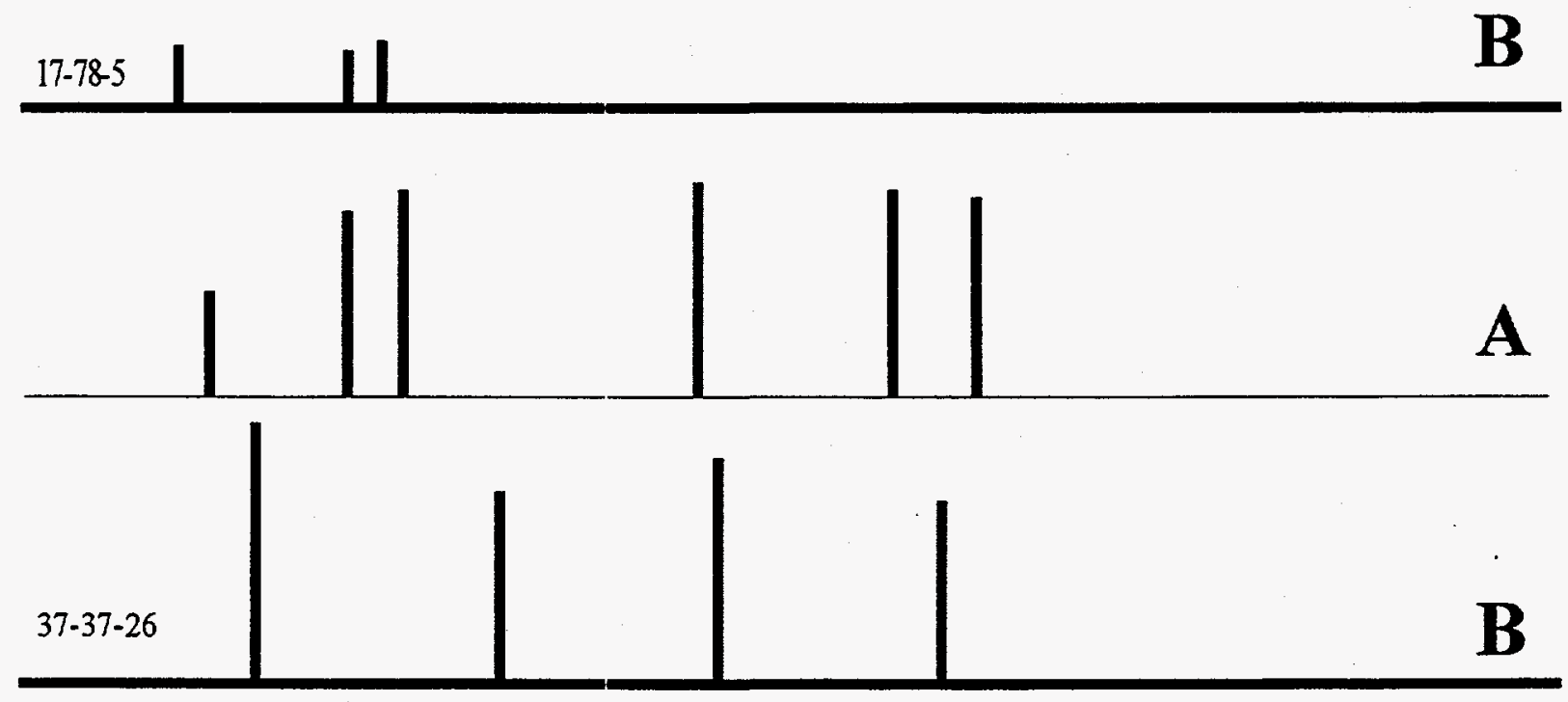

$74-2-24$

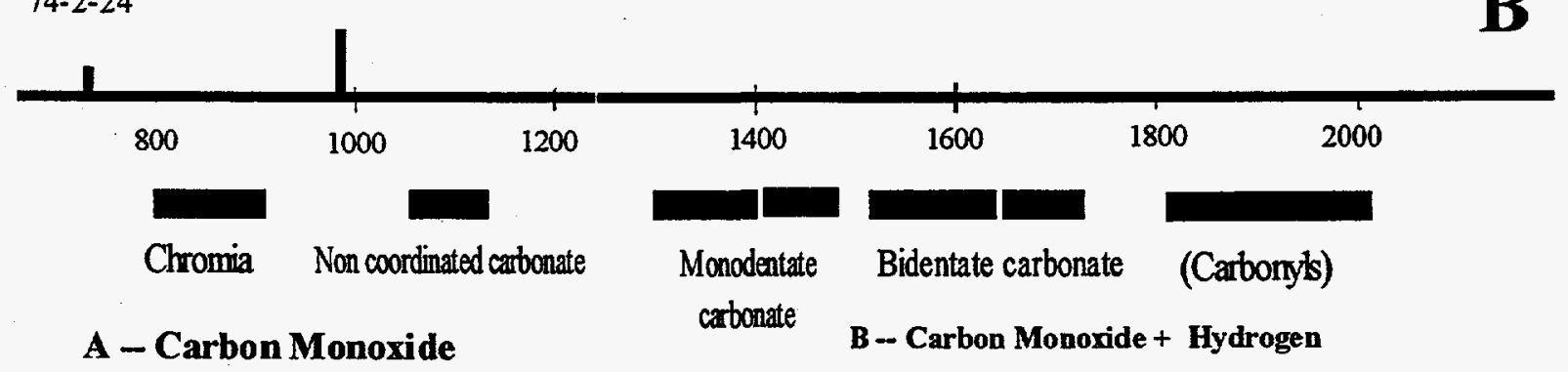


Table 12: Vibrational Frequencies of $\mathrm{CO}$ and $\mathrm{CO}+\mathrm{H}_{2}$ on $\mathrm{Cu}-\mathrm{CO}-\mathrm{Cr} \mathrm{C}$ atalys ts Assign-

\begin{tabular}{|c|c|c|c|c|c|c|c|c|c|c|c|c|}
\hline \multicolumn{4}{|c|}{$17-78-5$} & \multicolumn{4}{|c|}{$37-37.26$} & \multicolumn{4}{|c|}{$74-2-24$} & ment \\
\hline $\begin{array}{l}\text { With } \\
\text { co }\end{array}$ & esorbec & $\begin{array}{c}\mathrm{CO}+ \\
\mathrm{H}_{2}\end{array}$ & Desorbed & With co & Desorbed & $\begin{array}{c}\mathrm{CO}+ \\
\mathrm{H}_{2}\end{array}$ & esorbed & $W$ ith $c 0$ & esorbed & $\begin{array}{c}\mathrm{CO}+ \\
\mathrm{H}_{2}\end{array}$ & Desorbed & \\
\hline 1954 & - & - & & & $\dot{-}$ & & & 1931 & - & - & - & $\begin{array}{l}\text { Carbonyls } \\
\left(\mathrm{CO}_{4}\right)\end{array}$ \\
\hline - & & - & & - & - & & - & - & & - & & $\begin{array}{c}\text { Carbonyls } \\
\left(\mathrm{CO}_{4}\right)\end{array}$ \\
\hline 1735 & - & - & - & - & - & - & - & - & - & - & - & $\begin{array}{c}\text { Carbonyls } \\
\left.\left(\mathrm{CO}_{4}\right)^{\circ}\right)\end{array}$ \\
\hline & 1002 & $\dot{-}$ & & & $1 \leq 24$ & & & 1072 & & & & $\begin{array}{c}v c=0 \\
\text { ivis }\end{array}$ \\
\hline 1585 & & - & & 1523 & 1542 & 1599 & 1579 & 1563 & 1587 & 1535 & & $\begin{array}{c}\mathrm{VCO}=0 \\
\mathrm{a}\end{array}$ \\
\hline 1474 & 1450 & 1428 & & & & & & & & 1464 & & $\begin{array}{c}v_{\alpha \sigma} \mathrm{CO}_{3}^{2-} \\
11\end{array}$ \\
\hline 1380 & & & & 1326 & 1349 & 1331 & 1353 & 1354 & 1326 & & & $\begin{array}{c}v_{0} c 00^{\circ} \\
\quad I I I\end{array}$ \\
\hline 1276 & & & & & & & & & & & & \\
\hline 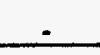 & 1178 & . & & 1185 & & & 1127 & - & & & & \\
\hline 1073 & & 1024 & 1019 & 1022. & 1034 & & & 1018 & & & & $\begin{array}{c}\mathrm{v}, \mathrm{CO}_{3}{ }^{2} \mathrm{ve}-\mathrm{O} \\
11 / 111\end{array}$ \\
\hline - & 989 & - & 981 & & 980 & & - & 923 & 936 & 963 & 983 & $\mathrm{CO}$ on $\mathrm{Cr}_{2} \mathrm{O}_{3}$ \\
\hline & & 823 & 822 & 837 & 844 & 896 & 885 & - & & & & \\
\hline & & & & - & - & & . & . & & 732 & 733 & \\
\hline & & & & 691 & & & & & & 698 & & \\
\hline
\end{tabular}




\section{REFERENCES}

1. Anderson, E.V. Chem. \& Eng. News 18, April (1986).

2. Hirsch, R L. Science 235,1467 (1987).

3. Von der Decker, C.B. Fedders, H. and Hohlein, B. Proc. VI th Int. Symp. on Alcohol Fuels Technology, Ottawa, Canada, May 21-25 (1984).

4. Schneider M.K., Kochloefl O. Bock., (Sud Chemie) EP-0152809-A2, (1985).

5. Ichikawa M., Bull. Chem. Soc. Japan, 51, 2268, 2273 (1978).

6. Kinkade N.E., (Union Carbide Corporation) EP-0149255 (1985).

7. Quardere G.J., G.A. Cochran (DOW Chemical) EP-0119609-Al (1984).

8. Institute Francois Du Petrole. UK Patents: GB 2118061A (1983), GB 2158730A (1985), U.S. Patent 4,291,126 (1981).

9. Xu Xiaoding, I.B., M. Doesburg and J.J F. Scholten., Catalysis Today 2, 125 (1987).

10. Marvin L. Cohen and Walter D. Knight., Physics Today 42-50, December 1990.

11. Gossard, A.C., and A.M. Portis, Suppl. J. Appl. Phys., 31, 205 (1960).

12. Selwood, P.W. "Magneto Chemistry", Inter. Sci. Publishers. (1956), "Chemisorption and Magnetization", Academic Press, New York (1975).

13. Wohlforth, E. P., "Ferromagnetic Materials", North Holland (1980) and D.J. Craik, "Magnetic Oxides", Wiley, New York (1975).

14. Bean, C.P., and J.D. Livingston, J.Appl. Phys., 30, 1205 (1959).

15. Luborsky, F.E., J. Appl. Phys. 33, 1909 (1959).

16. Candela, G.A., R.A. Haines Appl. Phys. Lelt. 34, 868 (1979).

17. Kneller Eckart "Fine Particle Theory - in Magnetism and Metallurgy" (Editor) Ami. Berkowitz, Academic Press 365-471.

18. Murty, A.N., A.A. Williams, R.T. Obermyer, and V.U.S. Rao, J. Appl. Phys., 61,4361 (1987).

19. Murty, A.N., A.A. Williams, R.T. Obermyer, V.U.S. Uao, and R.J. Gomley. Surface Science and Catalysis (1987) (Ed. J.W. Ward) PP 73-80, Elsevier Science Publishers, B.V. Amsterdam (1988). 
20. Murty, A.N., M. Seamster, A.N. Thorpe, R.T. Obermyer, V.U.S. Rao. J. Appl. Phys. $\underline{67}$, $5847,(1990)$.

21. Murty, A.N. "New Method of Magnetic Characterization of Zeolite Cobalt Catalysts"Final Technical Report U.S. D.O.E. Grant DE-FG22-85PC 80536 Aug 31, (1988).

22. Murty, A.N. "NMR Investigation of Supported Metal Catalysts for Syngas Conversion" Final Technical Report. U.S. DO.E. Grant DE-FG22-87PC-79917. Nov 1, 1990

23. Murty, A.N., Donatto, U.A., Washington, J.W. Hoard, T.L., Akundi, M.A., and Harris, C., IEEE Transactions on Magnetics, $\underline{30}, 4722,1994$

24. Murty, A.N., "NMR - NQR studies of Higher Alcohol Synthesis Cu-Co Catalysts", Final Technical Report U.S. D.O.E. Grant DE-FG22-89PC8976 (M003), March 1995.

25. Zafiris, G.S., and R.J. Gorte, J. Cat. 132, 275, (1991).

26. Decanio, E.C., and D.A. Storm, J. Cat. 132, 275, 1991.

27. Yan Y., Q. Xin, S. Jiang and X. Guo, J. Cat., 131, 234, (1991).

28. Balliard-Letrournel, R.M., Gomez Cobo, A.J., Mirodotos, C., Primet, M., and Dalmon, J.A., caatalysis Letters, $\underline{2}$ 149,(1989).

29. Mouaddib, N., Perrichon, V., and Primet, M., J. Chem. Soc.Farad. Trans. 85, 3413, (1989); Proc. $9^{\text {th }}$. Int. Congr. Catal. 2 , 521,(1988).

30. Akundi, M.A., D.V. K. Rao, and P.T. Rao., Proc. Roy. Ir. Academy, $\underline{\text { A73 }}$, 212, (1973).

31. Akundi, M.A., Putcha V., and Basu S.K., Ind. J. Phys., 53B, 388, (1980).

32. Byung, KNA, Walters, BA, and Vannice, M.A., J.Catal, 140, 585(1993).

33. Hamedh, M.I, and King D ., J. catal, 88, 264(1972).

34. Venter, J.J and Vannice, M.A. ., Appl. Spectrosc, 42, 1096 (1988).

35. Venter J.J and Vannice, M.A., J. Am.Chem.Soc III, 2377(1989) 
36. Augustine S.M, and Blitz, J.P., J. Catal, 142, 312(1993).

37. Alerasool, S., and Gonzalez, R.D., J. Catal. 124, 204 (1990).

38. Courty, P., Arlie J.P., Convers A., Mikitenkop P., and Sugier, A., Hydrocarbon Processing 105, (1984).

39. Courty, P., Durand D., Freund E., and Sugier A., J. Mol. Catalysis 17, 241 (1982).

40. Toth, L.E., and Ravitz, S.F., J. Phys. Chem., Solids 24, 1203-12076, 1963

41. Davydov, A.A. "Infrared Spectroscopy of Adsorbed Species on the Surface of Transiton Metal Oxides." John Wiley \&. sons,(1990)

42. Ishi, S., Ohno, Y., and Viswanathan, B., Surf. Sci., 61,349 (1985)

43. Broden, G., Rhodin, T.N., Bruckner, C.F., Bendon, R., and Hurych, Z., Surf. Sci., 59, 593 (1976).

44. Hinderman, J.P., Hutchings, G.T., and Kiennemann, A., Catal. Rev. - Sci. Eng 35, 1-127, (1993).

45. Longoni, G., Chini, P., Martinengo, S. Cavallieri, A., Calabressi J., and Dahl L., VI Conveg., "Nazionale di, Chemica Inorganica," paper A27, 1973.

46. Dalmon, J., Primet M., Martin, A., and Imelik, B., Surface Sci., 50, 95 (1975).

47. Mohan Rao, K., Scareno, D., Spoto, A., Zecchina, A., Surface Sci., 204, 319 (1988).

48. Nakamoto, K. "Infrared Sprectra of Inorganic and Coordination Compounds (in Russian)." Mir, Moscow 1966, John Wiley, New York (1975). 


\section{MINORITY UNDERGRADUATE STUDENT TRAINING: PAPERS PRESENTED}

"NMR and Magnetization Studies oj syngas Conversion Catalysts" E.L. Bruster*, L.Turner*, T. Porter*, A.N. Murty, S. Davis*, C. Collier* and M.A. Akundi. $72^{\text {nd }}$ Annual Louisiana Academy of Sciences Meeting, Hammond, LA. February (1998).

"CO Adsorption on Cobalt Particles Supported on Chromia - An FTIR Study" C. Collier*, A. Tsega*, M.A. Akundi and A.N. Murty. Undergraduate Research Conference, University of Texas, Austin, TX April (1997).

"CO Adsorption Effect on the Magnetic Character of $\mathrm{Cu} / \mathrm{Co} / \mathrm{Cr}$ Composite Catalysts" G. Lampkin*, J. White*, M. White*, A.N. Murty, S. Davis*, C. Collier*, and M.A. Akundi. 71 Annual Louisiana Academy of Sciences Meeting, Alexandria, LA. February (1997).

"CO Adsorption Studies on Metal Precursors of Copper, Cobalt and Chromium" A. Tsega*, S. Davis*, and M.A. Akundi. $71^{\text {st }}$ Annual Louisiana Academy of Sciences Meeting, Alexandria, LA. February (1997).

"Magneto-Chemistry of Cu-Co-Cr. Catalysts" G. Lampkin*, J. White*, A.N. Murty, S. Davis*, C. Collier*, and M.A. Akundi. American Association Physics Teachers Conference, College Park, MD. August (1996).

"Magneto-Chemical Characteristic of CO Adsorbed Cu-Co-Cr Catalysts" G. Lampkin*, J. White*, M. White*, and A.N. Murty. $70^{\text {th }}$ Annual Louisiana Academy of Sciences Meeting, Thibodaux, LA. February (1996).

"Effect of Carbon Monoxide Adsorption on the Magneto-Chemical Character of Syngas Conversion Catalysts" S.Davis*, S. Downs*, M.A. Akundi, G. Lampkin*, J. White*, M. White*, and A.N. Murty. Proceedings of U.S. DOE Fourth-Annual HBCU/Private Sector/Fossil Energy Research and Development Technology Transfer Symposium, Greenboro, NC. April (1996).

"NMR and Magnetic Character Studies on CO Adsorbed Syngas Catalysts" S. Davis*, M.A. Akundi, G.Lampkin*, and A.N. Murty. National Conference on Diversity in the Scientific and Technological Workplace, Washington, D.C. September 21-23 (1995).

"Effect of Preparative Techniques on the Magnetic Characteristics of Syngas Conversion Catalysts" A.N. Murty, G. Lampkin*, J. White*, M. White* and M.A. Akundi. The U.S. DOE Third Annual HBCU/Private Sector/Fossil Energy Research and Development Technology Transfer Symposium, Atlanta, GA. April 27-29 (1995).

"Investigation of Magnetic Properties of Syngas Conversion Cu-Co-Cr Catalysts" A.N. Murty, J. White* ${ }^{*}$ M. White*, and G. Lamplin*. $69^{\text {th }}$ Louisiana Academy of Sciences Meeting, Ruston, LA. February (1995). 
"Magneto-Chemical Characteristics of Higher Alcohol Synthesis Cu-Co-Cr Catalysts" U. Donatto*, J.W. Washington*, T. Hoard*, A.N. Murty, C. Harris*, and M.A. Akundi. The $6^{\text {th }}$ Joint MMM-Intermag Conference, Albequerque, NM. June 20-24 (1994) (to be published in IEEE Transactions on Magnetics)

"Magnetic Character Studies of $\mathrm{Cu} / \mathrm{Co} / \mathrm{Cr}$ Catalysts: Effect of Method of Preparation" C. Harris*, L. Williams*, M.A. Akundi, G. Lampkin*, C. Reed*, and A.N. Murty. The U.S. DOE Second. Annual HBCU/Private Sector/Fossil Energy Research and Development Technology Transfer Symposium, Birminham, AL. April 18-22 (1994).

* Participating Undergraduate Student Paper Presenter 
COPIES OF ABSTRACTS AND PAPERS PUBLISHED

(1994 -1997)

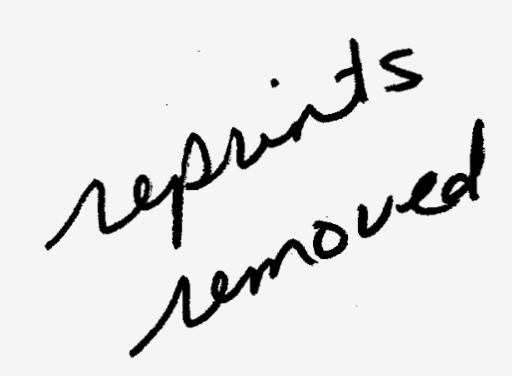

